\title{
Extremes of Markov-additive Processes with One-sided Jumps, with Queueing Applications
}

\author{
A. B. Dieker • M. Mandjes
}

Received: 9 October 2007 / Revised: 23 February 2009 /

Accepted: 5 June 2009 / Published online: 10 July 2009

(C) The Author(s) 2009. This article is published with open access at Springerlink.com

\begin{abstract}
Through Laplace transforms, we study the extremes of a continuoustime Markov-additive process with one-sided jumps and a finite-state background Markovian state-space, jointly with the epoch at which the extreme is 'attained'. For this, we investigate discrete-time Markov-additive processes and use an embedding to relate these to the continuous-time setting. The resulting Laplace transforms are given in terms of two matrices, which can be determined either through solving a nonlinear matrix equation or through a spectral method. Our results on extremes are first applied to determine the steady-state buffer-content distribution of several single-station queueing systems. We show that our framework comprises many models dealt with earlier, but, importantly, it also enables us to derive various new results. At the same time, our setup offers interesting insights into the connections between the approaches developed so far, including matrix-analytic techniques, martingale methods, the rate-conservation approach, and the occupation-measure method. We also study networks of fluid queues, and show how the results on single queues can be used to find the Laplace transform of the steady-state buffer-content vector; it has a matrix quasi-product form. Fluid-driven priority systems also have this property.
\end{abstract}

Keywords Markov-additive processes · Fluctuation theory • Queueing networks

AMS 2000 Subject Classifications Primary 60K25; Secondary 90B05 - 60G15

\footnotetext{
A. B. Dieker

H. Milton Stewart School of Industrial and Systems Engineering,

765 Ferst Drive, NW Atlanta, GA 30332-0205, USA

e-mail: ton.dieker@isye.gatech.edu

M. Mandjes ( $\square)$

Korteweg-de Vries Institute for Mathematics, University of Amsterdam,

Science Park, 9041098 XH Amsterdam, The Netherlands

e-mail: m.r.h.mandjes@uva.nl
} 


\section{Introduction}

A classical result, playing a pivotal role in applied probability, is what could be called the 'generalized Pollaczek-Khinchine formula': with $\bar{X}$ the supremum of a spectrally positive Lévy process $X$ (i.e., a Lévy process with no negative jumps) and with $\bar{F}^{X}$ the epoch at which this supremum is (first) 'attained', under the assumption of a negative drift $\mathrm{E} X(1)<0$,

$$
\mathrm{E} e^{-\alpha \bar{F}^{X}-\beta \bar{X}}=-\mathrm{E} X(1) \frac{\beta-\Phi_{-X}(\alpha)}{\psi_{-X}(\beta)-\alpha},
$$

for $\alpha, \beta \geq 0$ with $\psi_{-X}(\beta) \neq \alpha$; see for instance (Bertoin 1996, Thm. VII.4). In this formula, $\psi_{-X}(\beta):=\log \mathrm{E} e^{-\beta X(1)}$ is the Laplace exponent of $-X$, and $\Phi_{-X}$ is its inverse (which exists since $\psi_{-X}$ increases on $[0, \infty)$ ). Exploiting an equality in law between $\bar{X}$ and the the steady-state buffer content in an M/G/1 queue (the buffercontent process can be thought of as being obtained from $X$ by Skorokhod reflection at 0$)$, Eq. 1 also provides us with the Laplace-transform of the steady-state buffer content in the system - note that by taking $\alpha=0$ and assuming that the Lévy process is of compound-Poisson type, we retrieve the classical Pollaczek-Khinchine formula. This explains why the above framework is one of the cornerstones of queueing theory, but also of application domains where key performance measure can be expressed in terms of extremes, such as risk theory and mathematical finance.

There are several directions in which one could extend Eq. 1. This paper addresses two such extensions. (A) In the first place, our paper covers a generalization in which $X$ corresponds to a spectrally positive Markov-additive process; such a process can be thought of as a Markov-modulated Lévy process (with additional jumps at transitions of the background process). (B) In the second place, motivated by the aforementioned relationship between fluid queues and extremes, we extend these results to networks of fluid queues. Specifically, the contributions of this paper are the following:

A. One of the motivations for the present paper is to find an analog of Eq. 1 for spectrally positive Markov-additive processes with finitely many background states. The quantity $\Phi_{-X}(\alpha)$ becomes a matrix in the Markov-additive setting, and it is a key challenge to describe this matrix in the current general setting. The present paper is the first to achieve this goal in full generality through novel insights relying on a spectral method, which is complemented by a corresponding formula for the infimum of $X$.

The derivation of our results relies on Wiener-Hopf theory for an embedded process, in conjunction with a ladder height analysis as in Feller (1971, Ch. XII). Perhaps for historic reasons, the Wiener-Hopf technique is sometimes regarded as a complex-analysis tool from which probabilistic insight cannot be obtained. However, inspired by the work of Kennedy (1994), we are able to give appealing interpretations of all our results in terms of a last-passage process. Our approach to Markov-additive processes is essentially different from the occupation method developed in Asmussen (2000), the martingale method of Asmussen and Kella (2000), and the rate-conservation method of Miyazawa (2004). 
On the technical level, two steps are crucial. In the first place, we convert our continuous-time process to a discrete-time Markov-additive process by using an embedding. The maximum of the original, continuous-time process coincides with the maximum of the embedded process. In the special case of continuous Markov-additive processes, this idea has been applied by Asmussen (1995). However, by using this embedding we lose information on the epoch at which the extreme is 'attained', and we therefore also apply a second idea: we impose a step-dependent killing mechanism through which we keep track of the 'time' that passes in the continuous-time process between embedding epochs. The resulting procedure enables us to find the counterpart of Eq. 1. We remark that the killing technique is an alternative to other approaches that have been proposed for fluid-flow models (Ahn and Ramaswami 2005; Asmussen 1994; Bean et al. 2005b).

Our results for discrete-time processes are of independent interest; they unify and extend (parts of) Section 1.12 and Chapter 5 of Prabhu (1998). We exemplify this by analyzing a ramification of a queueing system with Markovmodulated ON/OFF input introduced by Cohen (1974); although this input does not fall into the framework of Markov-additive processes, we can still analyze its buffer-content distribution using our results on discrete-time processes. As a further application, we show that our approach may also be useful when the number of background states is infinite; a specific contribution of our work is a procedure to determine the steady-state distribution of the $\mathrm{M} / \mathrm{M} / \infty$-driven fluid queue.

B. A second motivation for this paper was a procedure, developed by Dębicki et al. (2007) for Lévy-driven tandem queueing systems, which expresses the Laplace transform of the joint buffer-content distribution in terms of the corresponding Laplace transform Eq. 1 for a single queue. Our main contribution here is that we show how this translation can be performed in a Markov-additive setting, by converting the counterpart of Eq. 1 to the Laplace transform of the buffercontent vector in tandem networks with Markov-additive input. This part of our work extends the results found by Dȩbicki et al. (2007) and Kella and Whitt (1992b) on tandem networks with Lévy input and the results by Kella (2001) on networks with deterministic background-dependent input rates.

Although we give matrix equations for all matrices that play an important role in the theory, it is still an interesting and challenging issue to devise efficient algorithms for numerically calculating these matrices. Therefore, our work could accelerate the development of such new numerical methods. We find this indispensable for a successful application of the theory.

This paper is organized as follows. First, in Section 2, we start with the analysis of the extremes of a discrete-time Markov-additive process. The insights that we obtain are then applied to continuous-time Markov-additive processes in Section 3. Section 4 casts our results on extremes into the setting of single-station queues, and some examples are given in Section 5. In Section 6 we show how these results on single queues can be used to determine the Laplace transform of the steady-state 
buffer-content vector in tandem fluid networks, and we conclude the paper with some extensions of our theory (Section 7).

\section{A Discrete-time Process and its Extremes}

This section introduces the discrete-time three-dimensional process $(S, T, J)=$ $\left\{\left(S_{n}, T_{n}, J_{n}\right): n \geq 0\right\}$. Although this process may look quite specific at first sight, we show in Sections 4-7 that it is highly versatile: it can be used to study the steadystate buffer content (in conjunction with the steady-state age of the busy period) for a broad class of queueing systems, including networks and priority queues.

\subsection{Definitions and Assumptions}

The discrete-time process $(S, T, J)$ takes values in $\mathbb{R} \times \mathbb{R}_{+} \times \mathcal{I}$, where $\mathcal{I}$ is a finite set with $N_{+}+N_{-}$elements. We write $\mathcal{I}_{+}$for the first $N_{+}$elements (which we call '+-points', as made clear below), and $\mathcal{I}_{-}$for the last $N_{-}$elements (which we call '--points'). The component $J$ is interpreted as a 'random environment'. We suppose that $(S, T, J)$ is defined on some measurable space $(\Omega, \mathcal{F})$.

Of primary interest is the minimum $\underline{S}$ and the maximum $\bar{S}$ of the process $S$. After setting $\underline{F}^{S}:=\inf \left\{n \geq 0: S_{n}=\inf _{k \geq 0} S_{k}\right\}$ and $\bar{F}^{S}:=\inf \left\{n \geq 0: S_{n}=\sup _{k \geq 0} S_{k}\right\}$, these are defined as $\underline{S}:=S_{F}$ and $\bar{S}:=S_{\bar{F}}$ respectively. The process $T$ is interpreted as the 'real' time that passes between the (discrete) time epochs; it cannot decrease. Therefore, it is also of interest to study $\underline{T}:=T_{F^{s}}, \bar{T}:=T_{\bar{F}} s, \underline{J}:=J_{F^{s}}$ and $\bar{J}:=J_{\bar{F}} s$. The aim of this section is to fully characterize the joint distributions of the triplet $(\underline{S}, \underline{T}, \underline{J})$ if $S$ drifts to $+\infty$, and $(\bar{S}, \bar{T}, \bar{J})$ if $S$ drifts to $-\infty$, under a measure specified below.

Let $\mathbb{P}$ be a probability measure on $(\Omega, \mathcal{F})$ (with corresponding integration operator $\mathbb{E}$ ) such that $(S, T, J)$ is a (discrete-time) Markov process on $\mathbb{R} \times \mathbb{R}_{+} \times \mathcal{I}$ under $\mathbb{P}$ with transition kernel given by

$$
p((s, t, j),(s+d v, t+d w, k))=\left\{\begin{array}{l}
p_{j k}^{J} \mathbb{P}\left(U^{j k} \in d v, \sigma^{j k} \in d w\right) \text { if } j \in \mathcal{I}_{+}, k \in \mathcal{I} \\
p_{j k}^{J} \mathbb{P}\left(-D^{j} \in d v, \tau^{j} \in d w\right) \text { if } j \in \mathcal{I}_{-}, k \in \mathcal{I}
\end{array}\right.
$$

where the $\sigma^{j k}, U^{j k}, \tau^{j}, D^{j}$ are random variables on $(\Omega, \mathcal{F})$. The $p_{j k}^{J}$ constitute the Markov transition matrix $\boldsymbol{P}^{J}$ of $J$ under $\mathbb{P}$, assumed to be irreducible. The unique stationary distribution of $J$ is written as $\pi_{J}$. We also assume that the $\mathbb{P}$-distributions of the vectors $\left\{\left(\sigma^{j k}, U^{j k}\right): j \in \mathcal{I}_{+}, k \in \mathcal{I}\right\}$ and $\left\{\left(\tau^{j}, D^{j}\right): j \in \mathcal{I}_{-}\right\}$are concentrated on $[0, \infty)^{2}$ and $[0, \infty) \times(0, \infty)$, respectively. The letters $U$ and $D$ stand for 'up' and 'down'. The $U^{j k}$ and $-D^{j}$ can be interpreted as 'jump sizes', whereas the $\sigma^{j k}$ and $\tau^{j}$ reflect 'sojourn times'. Note that $\mathbb{P}\left(\sigma^{j k}=0\right), \mathbb{P}\left(U^{j k}=0\right)$, and $\mathbb{P}\left(\tau^{j}=0\right)$ are allowed to be strictly positive.

For $k \in \mathcal{I}$, we write $\mathbb{P}_{k}$ for the law of $(S, T, J)$ given $S_{0}=T_{0}=0$ and $J_{0}=k$. To avoid trivialities, we suppose throughout that both $N_{-}$and $N_{+}$are nonzero, and that not all of the $U^{j k}$ are degenerate at zero. The following assumption is crucial in our analysis. 
Assumption 2.1 For any $j \in \mathcal{I}_{-}$, there exists some $\lambda_{j}^{\alpha}>0, \mu_{j}^{\alpha} \in(0,1]$ such that

$$
\mathbb{E} e^{-\alpha \tau^{j}-\beta D^{j}}=\mu_{j}^{\alpha} \frac{\lambda_{j}^{\alpha}}{\lambda_{j}^{\alpha}+\beta}, \quad \alpha, \beta \geq 0,
$$

where $\mu_{j}^{0}=1$.

Assumption 2.1 can be thought of as (a generalized version of) a memoryless property for the distribution of the jump sizes and sojourn times in the --points. We suppose that this assumption holds throughout this section. Motivation for the specific form of the above assumption can be found in Section 3.2.

In many of the proofs in this section, an important role is played by a family of probability measures $\left\{\mathbb{P}^{\alpha}: \alpha \geq 0\right\}$ on $(\Omega, \mathcal{F})$. We let the $\mathbb{P}^{\alpha}$-distribution of $U^{j k}$ be defined in terms of $\mathbb{P}$ through $\mathbb{P}^{\alpha}\left(U^{j k} \in d v\right)=\mathbb{E}\left[e^{-\alpha \sigma^{j k}} ; U^{j k} \in d v\right]$; this distribution is thus potentially defective. Similarly, we set $\mathbb{P}^{\alpha}\left(D^{j} \in d v\right)=\mathbb{E}\left[e^{-\alpha \tau^{j}} ; D^{j} \in d v\right]$. Furthermore, we let $(S, J)$ be a discrete-time Markov process under $\mathbb{P}^{\alpha}$ with transition kernel

$$
p^{\alpha}((s, j),(s+d v, k))=\left\{\begin{array}{l}
p_{j k}^{J} \mathbb{P}^{\alpha}\left(U^{j k} \in d v\right) \text { if } j \in \mathcal{I}_{+}, k \in \mathcal{I} \\
p_{j k}^{J} \mathbb{P}^{\alpha}\left(-D^{j} \in d v\right) \text { if } j \in \mathcal{I}_{-}, k \in \mathcal{I} .
\end{array}\right.
$$

The $\mathbb{P}^{\alpha}$-law for which $S_{0}=0$ and $J_{0}=k$ is denoted by $\mathbb{P}_{k}^{\alpha}$.

We note that $\left\{\left(S_{n}, J_{n}\right): n \geq 0\right\}$ is a discrete-time Markov-additive process under each of the measures $\mathbb{P}_{k}, \mathbb{P}_{k}^{\alpha}$ for $k \in \mathcal{I}$ and $\alpha \geq 0$. As a result, the powerful WienerHopf factorization for these processes is available. More details can be found in Arjas and Speed (1973) and Asmussen (2003, Sec. XI.2.2f). As an aside, we mention that $(S, T)$ can be interpreted as a two-dimensional additive component under $\mathbb{P}_{k}$; we do not use this.

In order to use the Wiener-Hopf technique, we need some more notation related to time-reversion. Let us therefore introduce the time-reversed transition probabilities

$$
\hat{p}_{j k}^{J}=\frac{\pi_{J}(k)}{\pi_{J}(j)} p_{k j}^{J}
$$

constituting the transition matrix $\widehat{\boldsymbol{P}}^{J}$; here $\pi_{J}(k)$ denotes the $k$-th element of $\boldsymbol{\pi}_{J}$. Let $\widehat{\mathbb{P}}$ be a probability measure on $(\Omega, \mathcal{F})$ (with expectation operator $\widehat{\mathbb{E}}$ ) such that $(S, T, J)$ is a Markov process with transition kernel

$$
\widehat{p}((s, t, j),(s+d v, t+d w, k))=\left\{\begin{array}{l}
\widehat{p}_{j k}^{J} \mathbb{P}\left(U^{k j} \in d v, \sigma^{k j} \in d w\right) \text { if } j \in \mathcal{I}, k \in \mathcal{I}_{+} ; \\
\widehat{p}_{j k}^{J} \mathbb{P}\left(-D^{k} \in d v, \tau^{k} \in d w\right) \text { if } j \in \mathcal{I}, k \in \mathcal{I}_{-} .
\end{array}\right.
$$

It is instructive to compare this 'time-reversed' kernel with the kernel $p$ defined above. The $\widehat{\mathbb{P}}$-law for which $S_{0}=T_{0}=0$ and $J_{0}=k$ is denoted by $\widehat{\mathbb{P}}_{k}$.

Finally, we also define the probability measures $\widehat{\mathbb{P}}^{\alpha}$ by requiring that $(S, J)$ is a Markov process with transition kernel

$$
\widehat{p}^{\alpha}((s, j),(s+d v, k))=\left\{\begin{array}{l}
\widehat{p}_{j k}^{J} \mathbb{P}^{\alpha}\left(U^{k j} \in d v\right) \text { if } j \in \mathcal{I}, k \in \mathcal{I}_{+} ; \\
\widehat{p}_{j k}^{J} \mathbb{P}^{\alpha}\left(-D^{k} \in d v\right) \text { if } j \in \mathcal{I}, k \in \mathcal{I}_{-},
\end{array}\right.
$$

and $\widehat{\mathbb{P}}_{k}^{\alpha}$ is defined as the $\widehat{\mathbb{P}}^{\alpha}$-law of this process given $S_{0}=0$ and $J_{0}=k$. 


\subsection{Notation}

We now introduce some convenient matrix notation. It is our aim to present a set of notation rules that we follow throughout the paper, as opposed to defining all vectors and matrices individually.

We start with our conventions for matrices. We define $\boldsymbol{A}_{++}, \boldsymbol{A}_{-+}, \boldsymbol{A}_{+-}, \boldsymbol{A}_{--}$for a given $(\mathcal{I} \times \mathcal{I})$-matrix $\boldsymbol{A}$ through its block form

$$
\boldsymbol{A} \equiv\left(\begin{array}{cc}
\boldsymbol{A}_{++} & \boldsymbol{A}_{+-} \\
\boldsymbol{A}_{-+} & \boldsymbol{A}_{--}
\end{array}\right),
$$

so that, for instance, $\boldsymbol{A}_{++}$is an $\left(\mathcal{I}_{+} \times \mathcal{I}_{+}\right)$-matrix. An example is the $(\mathcal{I} \times \mathcal{I})$-identity matrix, denoted by $\boldsymbol{I}$, which consists of the blocks $\boldsymbol{I}_{++}, \mathbf{0}_{+-}, \mathbf{0}_{-+}$, and $\boldsymbol{I}_{--}$in selfevident notation. The diagonal matrix with the vector $\boldsymbol{v}$ on its diagonal is written as $\operatorname{diag}(\boldsymbol{v})$. For example, $\boldsymbol{I}=\operatorname{diag}(\mathbf{1})$, where $\mathbf{1}$ stands for the $\mathcal{I}$-vector with ones. The vector with elements $\left\{\lambda_{j}^{\alpha}: j \in \mathcal{I}\right\}$ is written as $\operatorname{vec}\left(\lambda^{\alpha}\right)$, and $\operatorname{diag}\left(\lambda^{\alpha}\right)$ is shorthand for $\operatorname{diag}\left(\operatorname{vec}\left(\lambda^{\alpha}\right)\right)$. We also write $\operatorname{diag}\left(\lambda^{\alpha} /\left(\lambda^{\alpha}+i \beta\right)\right)$ for the $\left(\mathcal{I}_{-} \times \mathcal{I}_{-}\right)$-diagonal matrix with element $(j, j)$ equal to $\lambda_{j}^{\alpha} /\left(\lambda_{j}^{\alpha}+i \beta\right)$. Moreover, we write

$$
{ }^{\#} \boldsymbol{A}:=\operatorname{diag}\left(\boldsymbol{\pi}_{J}\right)^{-1} \boldsymbol{A}^{\prime} \operatorname{diag}\left(\boldsymbol{\pi}_{J}\right),
$$

where "' denotes matrix transpose. In conjunction with block notation, \# has priority over block notation: ${ }^{\#} \boldsymbol{A}_{++}$is the $(+,+)$-block of ${ }^{\#} \boldsymbol{A}$.

The second set of notation rules shows how vectors and matrices are built from probabilities and expectations involving a background process with values in $\mathcal{I}$. The resulting matrices or vectors are distinguished by writing $\boldsymbol{P}$ and $\boldsymbol{E}$ instead of $\mathbb{P}$ and $\mathbb{E}$, respectively, and by omitting indices. For instance, we set

$$
\boldsymbol{E}\left[S_{1} ; J_{1}\right]:=\left\{\mathbb{E}_{j}\left[S_{1} ; J_{1}=k\right]: j, k \in \mathcal{I}\right\},
$$

and the $j$-th element of the vector $\boldsymbol{E} S_{1}$ is $\mathbb{E}_{j} S_{1}$. Similarly, the $j$-th element of the vector $\boldsymbol{P}\left(S_{1}>0\right)$ is $\mathbb{P}_{j}\left(S_{1}>0\right)$. A matrix involving $\boldsymbol{P}$ or $\boldsymbol{E}$ can be partitioned into four blocks as described before, in which case a subscript ' - ' or ' + ' below $\boldsymbol{P}$ or $\boldsymbol{E}$ indicates the row of the matrix block, and a ' $\in+$ ' or ' $\in-$ ' after the background process indicates the column. For instance, the matrix $\boldsymbol{E}\left[S_{1} ; J_{1}\right]$ consists of four blocks, which we write as $\boldsymbol{E}_{+}\left[S_{1} ; J_{1} \in+\right], \boldsymbol{E}_{+}\left[S_{1} ; J_{1} \in-\right], \boldsymbol{E}_{-}\left[S_{1} ; J_{1} \in+\right]$, and $\boldsymbol{E}_{-}\left[S_{1} ; J_{1} \in-\right]$. The first row consists of two blocks and can be written as $\boldsymbol{E}_{+}\left[S_{1} ; J_{1}\right]$. Matrices such as $\widehat{\boldsymbol{E}}_{ \pm}\left[S_{1} ; J_{1}\right]$ are defined analogously, but with $\mathbb{E}_{j}$ replaced by $\widehat{\mathbb{E}}_{j}$. Similar conventions apply to vectors, which should always be interpreted as column vectors: the restriction of the vector $\boldsymbol{E} S_{1}$ to $\mathcal{I}_{+}\left(\right.$or $\mathcal{I}_{-}$) is written as $\boldsymbol{E}_{+} S_{1}$ (or $\left.\boldsymbol{E}_{-} S_{1}\right)$. Note that we have the relation $\boldsymbol{E}_{+} S_{1}=\boldsymbol{E}_{+}\left[S_{1} ; J_{1}\right] \mathbf{1}=\boldsymbol{E}_{+}\left[S_{1} ; J_{1} \in+\right] \mathbf{1}_{+}+$ $\boldsymbol{E}_{+}\left[S_{1} ; J_{1} \in-\right] \mathbf{1}_{-}$, where $\mathbf{1}_{+}$and $\mathbf{1}_{-}$stand for the $\mathcal{I}_{+}$-vector and $\mathcal{I}_{-}$-vector with ones, respectively. The $\mathcal{I}$-vector with zeroes is written as $\mathbf{0}$, and consists of $\mathbf{0}_{+}$and $\mathbf{0}_{-}$.

We now give examples of the above conventions for some quantities that play an important role in this paper. We set for $\alpha \geq 0, \beta \in \mathbb{R}$

$$
F_{j k}(\alpha, \beta):= \begin{cases}p_{j k}^{J} \mathbb{E} e^{-\alpha \sigma^{j k}+i \beta U^{j k}} & \text { if } j \in \mathcal{I}_{+}, k \in \mathcal{I} \\ p_{j k}^{J} \mathbb{E} e^{-\alpha \tau^{j}-i \beta D^{j}} & \text { if } j \in \mathcal{I}_{-}, k \in \mathcal{I} .\end{cases}
$$

This defines not only the matrix-transform of the transition kernel $\boldsymbol{F}(\alpha, \beta):=$ $\left\{F_{j k}(\alpha, \beta): j, k \in \mathcal{I}\right\}$, but also its four block matrices. Note that Assumption 2.1 
specifies the structure of $\boldsymbol{F}_{-+}(\alpha, \beta)=\boldsymbol{E}_{-}\left[e^{-\alpha T_{1}+i \beta S_{1}} ; J_{1} \in+\right]$ and $\boldsymbol{F}_{--}(\alpha, \beta)=$ $\boldsymbol{E}_{-}\left[e^{-\alpha T_{1}+i \beta S_{1}} ; J_{1} \in-\right]$. The time-reversed counterpart is written as $\widehat{\boldsymbol{F}}(\alpha, \beta)$, i.e., $\widehat{\boldsymbol{F}}(\alpha, \beta):={ }^{\#} \boldsymbol{F}(\alpha, \beta)$. Note that in particular $\widehat{\boldsymbol{P}}^{J}={ }^{\#} \boldsymbol{P}^{J}$. The identity ${ }^{\#} \widehat{\boldsymbol{F}}(\alpha, \beta)=$ $\boldsymbol{F}(\alpha, \beta)$ is frequently used in the sequel. Given $j \in \mathcal{I}_{+}$, we write $F_{j k}(\alpha, i \infty)$ for $\lim _{\beta \rightarrow \infty} F_{j k}(\alpha, i \beta)=p_{j k}^{J} \mathbb{E}\left[e^{-\alpha \sigma^{j k}} ; U^{j k}=0\right]$, thereby also defining $\boldsymbol{F}_{+-}(\alpha, i \infty)$ and $\boldsymbol{F}_{++}(\alpha, i \infty)$.

\subsection{The Ladder Heights of $S$}

The goal of this subsection is to characterize the $\mathbb{P}_{k}$-distribution of $(S, T, J)$ at the first strict ascending ladder epoch of $S$ and at its first strict descending ladder epoch. We do not impose conditions on the drift of $S$ yet.

The first strict ascending ladder epoch and the first weak descending ladder epoch of $S$ are defined as

$$
\tau_{+}=\inf \left\{n \geq 1: S_{n}>0\right\}, \quad \tau_{-}=\inf \left\{n \geq 1: S_{n} \leq 0\right\} .
$$

Its first strict descending ladder epoch, for which the weak inequality is replaced by a strict inequality, is denoted by $\tilde{\tau}_{-}$.

The Distribution of $\left(S_{\tau_{+}}, T_{\tau_{+}}, J_{\tau_{+}}\right)$In order to facilitate the investigation of the ascending ladder structure of $(S, T, J)$, we first prove a useful lemma related to $\tau_{-}$. For notational convenience, we define the matrix $\widehat{\boldsymbol{P}}^{\alpha}=\left\{\widehat{P}_{j k}^{\alpha}: j, k \in \mathcal{I}\right\}$ as

$$
\widehat{\boldsymbol{P}}^{\alpha}:=\widehat{\boldsymbol{E}}\left[e^{-\alpha T_{\tau_{-}}} ; J_{\tau_{-}}\right] .
$$

This matrix admits a block form as described in Section 2.2. A general remark is that, when integrating a defective random variable, we only carry out the integration over the set where the random variable is both finite and well-defined: in the above definition of $\widehat{\boldsymbol{P}}^{\alpha}$, it is tacitly assumed that $\tau_{-}<\infty$.

Lemma 2.1 Suppose that Assumption 2.1 holds. For $\alpha \geq 0, \beta \in \mathbb{R}$, we have

$$
{ }^{\#} \widehat{\boldsymbol{E}}\left[e^{-\alpha T_{\tau_{-}}+i \beta S_{\tau_{-}}} ; J_{\tau_{-}}\right]=\left(\begin{array}{cc}
\boldsymbol{F}_{++}(\alpha, i \infty) & \boldsymbol{F}_{+-}(\alpha, i \infty) \\
\operatorname{diag}\left(\frac{\lambda^{\alpha}}{\lambda^{\alpha}+i \beta}\right){ }^{\# \widehat{\boldsymbol{P}}_{-+}^{\alpha}} & \operatorname{diag}\left(\frac{\lambda^{\alpha}}{\lambda^{\alpha}+i \beta}\right){ }^{\# \widehat{\boldsymbol{P}}_{--}^{\alpha}}
\end{array}\right)
$$

Proof After recalling that $\tau_{-}$is a weak ladder epoch, it is immediate that for $\alpha \geq 0$, $j \in \mathcal{I}, k \in \mathcal{I}_{+}$,

$$
\widehat{\mathbb{E}}_{j}\left[e^{-\alpha T_{\tau_{-}}+i \beta S_{\tau_{-}}} ; J_{\tau_{-}}=k\right]=\widehat{p}_{j k}^{J} \mathbb{E}\left[e^{-\alpha \sigma^{k j}} ; U^{k j}=0\right]=\widehat{F}_{j k}(\alpha, i \infty) .
$$

Hence, it remains to calculate

$$
\widehat{\boldsymbol{E}}\left[e^{-\alpha T_{\tau_{-}}+i \beta S_{\tau_{-}}} ; J_{\tau_{-}} \in-\right]=\widehat{\boldsymbol{E}}^{\alpha}\left[e^{i \beta S_{\tau_{-}}} ; J_{\tau_{-}} \in-\right] .
$$

To find an expression for this quantity, we directly apply the idea of Lemma VIII.5.1 of Asmussen (2003), as follows. Evidently, for $j \in \mathcal{I}, k \in \mathcal{I}_{-}$, we have

$$
\widehat{\mathbb{P}}_{j}^{\alpha}\left(S_{\tau_{-}}<-x, J_{\tau_{-}}=k\right)=\sum_{n=1}^{\infty} \widehat{\mathbb{P}}_{j}^{\alpha}\left(S_{\tau_{-}}<-x, \tau_{-}=n, J_{\tau_{-}}=k\right) .
$$


Conditioning on $S_{n-1}$ and using Assumption 2.1, we see that the summands equal

$\widehat{\mathbb{E}}_{j}^{\alpha}\left[\mu_{k}^{\alpha} e^{-\lambda_{k}^{\alpha}\left(x+S_{n-1}\right)} ; \tau_{-}>n-1, J_{\tau_{-}}=k\right]=e^{-\lambda_{k}^{\alpha} x} \widehat{\mathbb{E}}_{j}^{\alpha}\left[\mu_{k}^{\alpha} e^{-\lambda_{k}^{\alpha} S_{n-1}} ; \tau_{-}>n-1, J_{\tau_{-}}=k\right]$,

since the value of the $n$-th increment should (in absolute terms) be larger than $x+$ $S_{n-1}$. Importantly, this is exponential in $x$, so that we obtain

$$
\widehat{\mathbb{E}}_{j}^{\alpha}\left[e^{i \beta S_{\tau_{-}}} ; J_{\tau_{-}}=k\right]=\frac{\lambda_{k}^{\alpha}}{\lambda_{k}^{\alpha}+i \beta} \sum_{n=1}^{\infty} \widehat{\mathbb{E}}_{j}^{\alpha}\left[\mu_{k}^{\alpha} e^{-\lambda_{k}^{\alpha} S_{n-1}} ; \tau_{-}>n-1, J_{\tau_{-}}=k\right] .
$$

The latter sum is calculated by inserting $\beta=0$ into this identity.

The above lemma requires knowledge of (submatrices of) ${ }^{\#} \widehat{\boldsymbol{P}}^{\alpha}$. The following proposition gives a fixed-point equation for these matrices, so that they can be found numerically. Write $\boldsymbol{F}_{++}^{\alpha}(d x)$ for the measure-valued $\left(\mathcal{I}_{+} \times \mathcal{I}_{+}\right)$-matrix with element $(j, k)$ equal to $p_{j k}^{J} \mathbb{P}^{\alpha}\left(U^{j k} \in d x\right)$ for $j, k \in \mathcal{I}_{+}$, and define $\boldsymbol{F}_{+-}^{\alpha}(d x)$ similarly.

Proposition 2.1 For $\alpha \geq 0$, we have

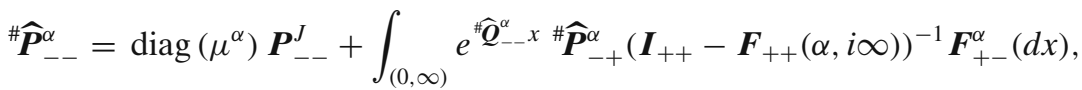

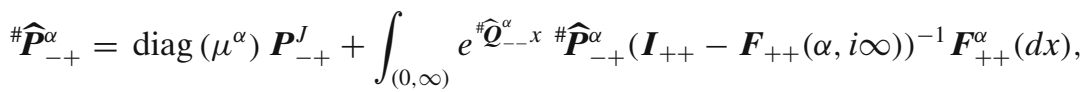

where integration should be understood as componentwise integration, and ${ }^{\#} \widehat{\boldsymbol{Q}}_{--}^{\alpha}$ is specified by

$$
{ }^{\#} \widehat{\boldsymbol{Q}}_{--}^{\alpha}=-\left[\boldsymbol{I}_{--}-{ }^{\#} \widehat{\boldsymbol{P}}_{-+}^{\alpha}\left(\boldsymbol{I}_{++}-\boldsymbol{F}_{++}(\alpha, i \infty)\right)^{-1} \boldsymbol{F}_{+-}(\alpha, i \infty)-{ }^{\# \widehat{\boldsymbol{P}}_{--}^{\alpha}}\right] \operatorname{diag}\left(\lambda^{\alpha}\right) .
$$

Proof Write $\tau_{-}(x):=\inf \left\{n>0: S_{n} \leq-x\right\}$ for $x \geq 0$. For $j \in \mathcal{I}$ and $k \in \mathcal{I}_{-}$, we have by the Markov property

$$
\widehat{P}_{j k}^{\alpha} \equiv \widehat{\mathbb{P}}_{j}^{\alpha}\left(J_{\tau_{-}}=k\right)=\widehat{p}_{j k}^{J} \mu_{k}^{\alpha}+\sum_{\ell \in \mathcal{I}_{+}} \widehat{p}_{j \ell}^{J} \int_{(0, \infty)} \mathbb{P}^{\alpha}\left(U^{\ell j} \in d x\right) \widehat{\mathbb{P}}_{\ell}^{\alpha}\left(J_{\tau_{-}(x)}=k\right) .
$$

Note that the integration interval for $U^{\ell j}$ is $(0, \infty)$, because if $U^{\ell j}$ were 0 , then $J_{\tau_{-}}$ would be in $\mathcal{I}_{+}$. The claims follow after showing that

$$
\widehat{\mathbb{P}}_{\ell}^{\alpha}\left(J_{\tau_{-}(x)}=k\right)=\sum_{m \in \mathcal{I}_{+}} \widehat{F}_{\ell m}(\alpha, i \infty) \mathbb{P}_{m}^{\alpha}\left(J_{\tau_{-}(x)}=k\right)+\sum_{j \in \mathcal{I}_{-}} \widehat{\mathbb{P}}_{\ell}^{\alpha}\left(J_{\tau_{-}}=j\right)\left[e^{\widehat{\boldsymbol{Q}}_{--}^{\alpha} x}\right]_{j k},
$$

where

$$
\widehat{\boldsymbol{Q}}_{--}^{\alpha}=-\operatorname{diag}\left(\lambda^{\alpha}\right)\left[\boldsymbol{I}_{--}-\widehat{\boldsymbol{F}}_{-+}(\alpha, i \infty)\left(\boldsymbol{I}_{++}-\widehat{\boldsymbol{F}}_{++}(\alpha, i \infty)\right)^{-1} \widehat{\boldsymbol{P}}_{+-}^{\alpha}-\widehat{\boldsymbol{P}}_{--}^{\alpha}\right] .
$$

To this end, note that $\tau_{-}(x)$ is nondecreasing in $x$. The first-passage process $\left\{J_{\tau_{-}(x)}\right.$ : $x \geq 0$ \} given $J_{\tau_{-}}=j$ is a (defective) Markov process under $\widehat{\mathbb{P}}_{\ell}^{\alpha}$ with values in $\mathcal{I}_{-}$, cf. Assumption 2.1. It suffices to prove that $\widehat{\boldsymbol{Q}}_{--}^{\alpha}$ is its intensity matrix. For ease we first concentrate on the case for which the distributions of the $U^{j \ell}$ do not have an 
atom at zero. After an exponentially distributed time with parameter $\lambda_{j}^{\alpha}$, the firstpassage process then jumps to a --point $k \in \mathcal{I}_{-}$with probability $\widehat{P}_{j k}^{\alpha}$ (where $j=k$ is allowed). For the general case where $U^{j \ell}$ may have an atom at zero, we have to take into account the paths in which $S$ stays at the same level for a while before entering $k \in \mathcal{I}_{-}$. This procedure leads to the given intensity matrix.

Our next result is a nonlinear system for the matrix $\boldsymbol{K}_{--}^{\alpha}$, where

$$
\boldsymbol{K}_{--}^{\alpha}:=\operatorname{diag}\left(\lambda^{\alpha}\right)^{\#} \widehat{\boldsymbol{Q}}_{--}^{\alpha} \operatorname{diag}\left(\lambda^{\alpha}\right)^{-1} .
$$

Since $\widehat{\boldsymbol{Q}}_{--}^{\alpha}$ is the intensity matrix of the first-passage (Markov) process of the timereversed process as detailed in the proof of Proposition $2.1, \boldsymbol{K}_{--}^{\alpha}$ is the intensity matrix for the last-passage process of the original process. To state the nonlinear system, we define for $\beta \in \mathbb{R}$,

$$
\boldsymbol{F}_{+\circlearrowleft-}(\alpha, \beta):=\left(\boldsymbol{I}_{++}-\boldsymbol{F}_{++}(\alpha, \beta)\right)^{-1} \boldsymbol{F}_{+-}(\alpha, \beta),
$$

and $\boldsymbol{F}_{+\circlearrowleft_{-}}^{\alpha}(d x)$ is the measure for which $\beta \mapsto \boldsymbol{F}_{+\circlearrowleft_{-}}(\alpha, \beta)$ is the characteristic function. These notions relate to the increment in the 'vertical direction', when starting in a +-point, until the epoch that a --point is reached. For simplicity we only prove uniqueness if $S$ drifts to $+\infty$ or $-\infty$. We write

$$
\mathcal{H}_{+}=\left\{\begin{array}{l}
\{\beta \in \mathbb{C}: \mathfrak{R}(\beta)>0\} \text { if } \lim _{n \rightarrow \infty} S_{n}=+\infty \\
\{\beta \in \mathbb{C}: \mathfrak{R}(\beta) \geq 0\} \text { if } \lim _{n \rightarrow \infty} S_{n}=-\infty
\end{array}\right.
$$

Corollary 2.1 For $\alpha \geq 0$, the matrix $\boldsymbol{K}_{--}^{\alpha}$ solves the nonlinear system

$$
\boldsymbol{K}_{--}^{\alpha}+\operatorname{diag}\left(\lambda^{\alpha}\right)\left(\boldsymbol{I}_{--}-\operatorname{diag}\left(\mu^{\alpha}\right) \boldsymbol{P}_{--}^{J}\right)-\int_{[0, \infty)} e^{\boldsymbol{K}_{--}^{\alpha} x} \operatorname{diag}\left(\mu^{\alpha} \lambda^{\alpha}\right) \boldsymbol{P}_{-+}^{J} \boldsymbol{F}_{+{ }^{-}-}^{\alpha}(d x)=\mathbf{0}_{--} .
$$

The solution is unique within the class of matrices with eigenvalues in $\mathcal{H}_{+}$.

Proof The idea of the proof is to slightly modify the process without changing the (time-reversed) first-passage process (and thus $\boldsymbol{K}_{--}^{\alpha}$ ). Indeed, interpret subsequent +-points as a single +-point; one then obtains a different discrete-time process, with $\boldsymbol{F}_{+-}(\alpha, \beta)$ replaced by $\boldsymbol{F}_{+\circlearrowleft_{-}}(\alpha, \beta)$. Importantly, for this 'new' $J$ we have that $\boldsymbol{P}_{++}^{J}=\mathbf{0}_{++}$, so that ${ }^{\sharp} \widehat{\boldsymbol{P}}_{-+}^{\alpha}=\operatorname{diag}\left(\mu^{\alpha}\right) \boldsymbol{P}_{-+}^{J}$ by Proposition 2.1. The formula for ${ }^{\#} \widehat{\boldsymbol{Q}}_{--}^{\alpha}$ in this proposition then immediately leads to the desired matrix equation for $\boldsymbol{K}_{--}^{\alpha}$. The proof of uniqueness is deferred to the appendix, see Corollary 8.1.

It is interesting to observe that, according to Corollary 2.1 and its proof, we may 'lump' subsequent +-points and assume without loss of generality that $\boldsymbol{P}_{++}^{J}=\mathbf{0}_{++}$in order to calculate $\boldsymbol{K}_{--}^{\alpha}$. This lumping can also be used to compute ${ }^{\#} \widehat{\boldsymbol{P}}_{+-}^{\alpha}$ and ${ }^{\#} \widehat{\boldsymbol{P}}_{--}^{\alpha}$ with Proposition 2.1, but only for $\alpha=0$.

There are several ways to extract algorithms for determining ${ }^{*} \widehat{\boldsymbol{P}}_{+-}^{\alpha},{ }^{\#} \widehat{\boldsymbol{P}}_{--}^{\alpha}$, and $\boldsymbol{K}_{--}^{\alpha}$ from Proposition 2.1 and Corollary 2.1. For instance, Corollary 2.1 can be interpreted as a fixed-point equation $K_{--}^{\alpha}=\varphi\left(K_{--}^{\alpha}\right)$ for some matrix-function $\varphi$. This suggests to fix an initial matrix $\boldsymbol{K}_{--}^{\alpha, 0}$, and then use the recursion $\boldsymbol{K}_{--}^{\alpha, n+1}=$ $\boldsymbol{\varphi}\left(\boldsymbol{K}_{--}^{\alpha, n}\right)$ to characterize a sequence of matrices that converges to $\boldsymbol{K}_{--}^{\alpha}$. We refer to Asmussen (2000, Sec. VI.2), Miyazawa (2004, Sec. 3), or Takada (2001, Sec. 4) 
for further details on this technique. One difficulty that needs to be overcome is the calculation of matrix exponentials, see Moler and Van Loan (2003) for a survey of available methods. It is not our aim to devise fast algorithms for computing the matrix $\boldsymbol{K}_{--}^{\alpha}$, and we shall therefore not address these algorithmic properties here. An alternative method for determining $\boldsymbol{K}_{--}^{\alpha}$ (and thus ${ }^{*} \widehat{\boldsymbol{P}}_{+-}^{\alpha}$ and ${ }^{\#} \widehat{\boldsymbol{P}}_{--}^{\alpha}$ ) is discussed in the Appendix.

The next proposition characterizes the $\mathbb{P}_{k}$-distribution of $\left(S_{\tau_{+}}, T_{\tau_{+}}, J_{\tau_{+}}\right)$. The main ingredient is the celebrated Wiener-Hopf factorization.

Proposition 2.2 For $\alpha \geq 0, \beta \in \mathbb{R}$ with $(\alpha, \beta) \neq 0$, we have

$$
\boldsymbol{E}\left[e^{-\alpha T_{\tau_{+}}+i \beta S_{\tau_{+}}} ; J_{\tau_{+}}\right]=\boldsymbol{I}-\left(\boldsymbol{I}-{ }^{\#} \widehat{\boldsymbol{E}}\left[e^{-\alpha T_{\tau_{-}}+i \beta S_{\tau_{-}}} ; J_{\tau_{-}}\right]\right)^{-1}(\boldsymbol{I}-\boldsymbol{F}(\alpha, \beta)),
$$

where nonsingularity is implicit.

Proof Write $\widehat{\boldsymbol{G}}(\alpha, \beta):=\widehat{\boldsymbol{E}}\left[e^{-\alpha T_{\tau_{-}}+i \beta S_{\tau_{-}}} ; J_{\tau_{-}}\right]$. The statement is the Wiener-Hopf factorization (e.g., Asmussen 2003, Thm. XI.2.12) for the Markov-additive process $S$ under the measure $\mathbb{P}^{\alpha}$, provided $\boldsymbol{I}-{ }^{\#} \widehat{\boldsymbol{G}}$ is nonsingular. This requirement is equivalent to nonsingularity of $\boldsymbol{I}-\widehat{\boldsymbol{G}}$.

To see that this matrix is nonsingular, we exploit the fact that $\widehat{\boldsymbol{G}}_{j k}$ is the transform of a nonlattice distribution for $j \in \mathcal{I}, k \in \mathcal{I}_{-}$. Therefore, we have $\left|\widehat{\boldsymbol{G}}_{j k}(\alpha, \beta)\right|<\widehat{P}_{j k}^{0}$ for $(\alpha, \beta) \neq(0,0)$, see, e.g., Theorem 6.4 .7 of Chung (2001). As a result, $\boldsymbol{I}-\widehat{\boldsymbol{G}}$ is a strictly diagonally dominant matrix:

$$
\sum_{k \in \mathcal{I}}\left|\widehat{\boldsymbol{G}}_{j k}(\alpha, \beta)\right|<\sum_{k \in \mathcal{I}_{+}} \widehat{p}_{j k}^{J} \mathbb{P}\left(U^{k j}=0\right)+\sum_{k \in \mathcal{I}_{-}} \widehat{P}_{j k}^{0} \leq 1
$$

where the last inequality follows from the fact that $S_{\tau_{-}}$has a (possibly defective) distribution, see Lemma 2.1.

The Distribution of $\left(S_{\tilde{\tau}_{-}}, T_{\tilde{\tau}_{-}}, J_{\tilde{\tau}_{-}}\right)$We now turn to our second aim of this subsection, the characterization of the distribution of $\left(S_{\tilde{\tau}_{-}}, T_{\tilde{\tau}_{-}}, J_{\tilde{\tau}_{-}}\right)$. This turns out to be simpler than the analysis of $\left(S_{\tau_{+}}, T_{\tau_{+}}, J_{\tau_{+}}\right)$; particularly, Wiener-Hopf techniques are not required here. We omit all proofs, since similar arguments apply as before.

In the context of strict decreasing ladder heights, a prominent role is played by the matrix

$$
\boldsymbol{P}_{+-}^{\alpha}:=\boldsymbol{E}_{+}\left[e^{-\alpha T_{\tilde{t}_{-}}} ; S_{1}>0, J_{\tilde{\tau}_{-}-1} \in-\right]
$$

The indices in this expression should be compared to those in the definition of $\widehat{\boldsymbol{P}}^{\alpha}$. We also set

$$
\boldsymbol{P}_{+\circlearrowleft^{-}}^{\alpha}:=\left(\boldsymbol{I}_{++}-\boldsymbol{F}_{++}(\alpha, i \infty)\right)^{-1}\left[\boldsymbol{P}_{+-}^{\alpha} \operatorname{diag}\left(\mu^{\alpha}\right)^{-1}+\boldsymbol{F}_{+-}(\alpha, i \infty)\right] .
$$

The analog of Lemma 2.1 follows immediately from these definitions: for $\alpha \geq 0$, $\beta \in \mathbb{R}$, we have

$$
\boldsymbol{E}\left[e^{-\alpha T_{\tilde{\tau}_{-}}+i \beta S_{\tilde{\tau}_{-}}} ; J_{\tilde{\tau}_{-}}\right]=\left(\begin{array}{cc}
\boldsymbol{P}_{+\sigma_{-}}^{\alpha} \operatorname{diag}\left(\frac{\mu^{\alpha} \lambda^{\alpha}}{\lambda^{\alpha}+i \beta}\right) \boldsymbol{P}_{-+}^{J} & \boldsymbol{P}_{+\sigma_{-}^{-}}^{\alpha} \operatorname{diag}\left(\frac{\mu^{\alpha} \lambda^{\alpha}}{\lambda^{\alpha}+i \beta}\right) \boldsymbol{P}_{--}^{J} \\
\operatorname{diag}\left(\frac{\mu^{\alpha} \lambda^{\alpha}}{\lambda^{\alpha}+i \beta}\right) \boldsymbol{P}_{-+}^{J} & \operatorname{diag}\left(\frac{\mu^{\alpha} \lambda^{\alpha}}{\lambda^{\alpha}+i \beta}\right) \boldsymbol{P}_{--}^{J}
\end{array}\right) .
$$


We continue with a result in the spirit of Proposition 2.1, which can be proved along the same lines.

Proposition 2.3 For $\alpha \geq 0$, we have

$$
\boldsymbol{P}_{+-}^{\alpha}=\int_{(0, \infty)} \boldsymbol{F}_{++}^{\alpha}(d x) \boldsymbol{P}_{+{ }^{-}-}^{\alpha} e^{\boldsymbol{Q}_{--}^{\alpha} x} \operatorname{diag}\left(\mu^{\alpha}\right)+\int_{(0, \infty)} \boldsymbol{F}_{+-}^{\alpha}(d x) e^{\boldsymbol{Q}_{--}^{\alpha} x} \operatorname{diag}\left(\mu^{\alpha}\right),
$$

where $\boldsymbol{Q}_{--}^{\alpha}$ is specified by

$$
\boldsymbol{Q}_{--}^{\alpha}=-\operatorname{diag}\left(\lambda^{\alpha}\right)\left[\boldsymbol{I}_{--}-\operatorname{diag}\left(\mu^{\alpha}\right) \boldsymbol{P}_{--}^{J}-\operatorname{diag}\left(\mu^{\alpha}\right) \boldsymbol{P}_{-+}^{J} \boldsymbol{P}_{+\circlearrowleft-}^{\alpha}\right] .
$$

We next turn to the analog of Corollary 2.1, which can be proven along the same lines. When inspecting the differences between the two corollaries, we first note that they are remarkably similar. Whereas the $\boldsymbol{K}_{--}^{\alpha}$-matrices are always the first matrices in each of the terms, the $\boldsymbol{Q}_{--}^{\alpha}$-matrices always appear last. In the Appendix, we show that this has a specific reason. The claimed uniqueness follows from Corollary 8.1.

Corollary 2.2 For $\alpha \geq 0$, the matrix $\boldsymbol{Q}_{--}^{\alpha}$ solves the nonlinear system

$$
\boldsymbol{Q}_{--}^{\alpha}+\operatorname{diag}\left(\lambda^{\alpha}\right)\left[\boldsymbol{I}_{--}-\operatorname{diag}\left(\mu^{\alpha}\right) \boldsymbol{P}_{--}^{J}-\int_{[0, \infty)} \operatorname{diag}\left(\mu^{\alpha}\right) \boldsymbol{P}_{-+}^{J} \boldsymbol{F}_{+\circlearrowleft-}^{\alpha}(d x) e^{\boldsymbol{Q}_{--}^{\alpha} x}\right]=\mathbf{0}_{--} .
$$

The solution is unique within the class of matrices with eigenvalues in $\mathcal{H}_{+}$.

\subsection{The Distribution of $(\bar{S}, \bar{T}, \bar{J})$}

In this section, we study $\bar{S}$ (jointly with $\bar{T}, \bar{J}$ ), assuming that $S$ drifts to $-\infty$. In fact, throughout this subsection, we suppose that $\boldsymbol{\pi}_{J}^{\prime} \boldsymbol{E} S_{1}<0$. We remark that, with the only exception of Lemma 2.3, all the results also hold under the weaker assumption that $S$ drifts to $-\infty$. Our main tools are the ladder-height results obtained in the previous subsection.

The following theorem expresses the transform of $(\bar{S}, \bar{T}, \bar{J})$ in terms of the matrix characterized in Lemma 2.1 and the (still unknown) vector $\boldsymbol{P}(\bar{S}=0)$. Observe that the matrices ${ }^{\#} \widehat{\boldsymbol{P}}_{--}^{\alpha}$ and ${ }^{\#} \widehat{\boldsymbol{P}}_{-+}^{\alpha}$ required in Lemma 2.1 can be found with Proposition 2.1.

Theorem 2.1 For $\alpha \geq 0, \beta \in \mathbb{R}$ with $(\alpha, \beta) \neq(0,0)$, we have

$$
\boldsymbol{E}\left[e^{-\alpha \bar{T}+i \beta \bar{S}} ; \bar{J}\right]=(\boldsymbol{I}-\boldsymbol{F}(\alpha, \beta))^{-1}\left(\boldsymbol{I}-{ }^{\#} \widehat{\boldsymbol{E}}\left[e^{-\alpha T_{\tau_{-}}+i \beta S_{\tau_{-}}} ; J_{\tau_{-}}\right]\right) \operatorname{diag}(\boldsymbol{P}(\bar{S}=0)) .
$$

Proof By the Markov property, we have for $\alpha \geq 0$ with $(\alpha, \beta) \neq(0,0), \beta \in \mathbb{R}$,

$$
\begin{aligned}
\boldsymbol{E}\left[e^{-\alpha \bar{T}+i \beta \bar{S}} ; \bar{J}\right] & =\left(\boldsymbol{I}-\boldsymbol{E}\left[e^{-\alpha T_{\tau_{+}}+i \beta S_{\tau_{+}}} ; J_{\tau_{+}}\right]\right)^{-1} \operatorname{diag}\left(\boldsymbol{P}\left(\tau_{+}=\infty\right)\right) \\
& =(\boldsymbol{I}-\boldsymbol{F}(\alpha, \beta))^{-1}\left(\boldsymbol{I}-{ }^{\#} \widehat{\boldsymbol{E}}\left[e^{-\alpha T_{\tau_{-}}+i \beta S_{\tau_{-}}} ; J_{\tau_{-}}\right]\right) \operatorname{diag}(\boldsymbol{P}(\bar{S}=0)),
\end{aligned}
$$

where the second equality follows from Proposition 2.2. The nonsingularity of $\boldsymbol{I}$ $\boldsymbol{F}(\alpha, \beta)$ follows from (strict) diagonal dominance, cf. the proof of Proposition 2.2. 
There is a direct, insightful interpretation of Theorem 2.1 in terms of a last-passage process, which is used on several occasions in this paper, inspired by Kennedy's interpretation (Kennedy 1994) of the Wiener-Hopf factorization. First note that the theorem states that $\boldsymbol{E}\left[e^{-\alpha \bar{T}+i \beta \bar{S}} ; \bar{J}\right]$ equals

$$
\sum_{n=0}^{\infty} \boldsymbol{F}^{n}(\alpha, \beta) \operatorname{diag}(\boldsymbol{P}(\bar{S}=0))-\sum_{k=0}^{\infty} \boldsymbol{F}^{k}(\alpha, \beta)^{\sharp} \widehat{\boldsymbol{E}}\left[e^{-\alpha T_{\tau_{-}}+i \beta S_{\tau_{-}}} ; J_{\tau_{-}}\right] \operatorname{diag}(\boldsymbol{P}(\bar{S}=0)) .
$$

Clearly, the $n$-th summand in the first term can be interpreted as the transform of $\left(S_{n}, T_{n}, J_{n}\right)$ on the event $\left\{\sup _{m \geq n} S_{m}=S_{n}\right\}$. If the maximum is attained at $T_{n}$, this is precisely $\boldsymbol{E}\left[e^{-\alpha \bar{T}+i \beta \bar{S}} ; \bar{J}\right]$. However, if this is not the case, we have to subtract the contribution due to the fact that there is an $\ell<n$ for which $S_{\ell} \geq S_{n}$. In that case, write $S_{n}=S_{k}+\left(S_{n}-S_{k}\right)$, where $k=\sup \left\{\ell<n: S_{\ell} \geq S_{n}\right\}$, so that $n$ is now a socalled last-passage epoch for the process with $\left(k, S_{k}\right)$ as the origin. Looking backward in time, starting from $\left(n, S_{n}\right), k$ is a first weak descending ladder epoch. The argument is completed by exploiting the Markov property. Partitioning with respect to the lastpassage epoch is sometimes called the Beneš-method (Beneš 1963).

It is insightful to give the complete argument for $\alpha=0$ in formulas. The terms that need to be subtracted (because the maximum occurred earlier) are

$$
\begin{aligned}
& \sum_{n=0}^{\infty} \boldsymbol{E}\left[e^{i \beta S_{n}} ; \forall m \geq n: S_{m} \leq S_{n}, \exists m<n: S_{m} \geq S_{n}, J_{n}\right] \\
& \quad=\sum_{k=0}^{\infty} \sum_{n=k+1}^{\infty} \boldsymbol{E}\left[e^{i \beta S_{k}+i \beta\left(S_{n}-S_{k}\right)} ; \sup _{m \geq n} S_{m}=S_{n}, S_{k} \geq S_{n}, \sup _{k<\ell<n} S_{\ell}<S_{n}, J_{n}\right],
\end{aligned}
$$

where the equality is justified by the fact that the events are disjoint as a result of the partitioning with respect to the last-passage epoch. Now note that the double sum is indeed the second sum in Eq. 5 for $\alpha=0$.

Theorem 2.1 implies that, to compute $\boldsymbol{E}\left[e^{-\alpha \bar{T}+i \beta \bar{S}}\right]$, only the determination of the vector $\boldsymbol{P}(\bar{S}=0)$ is left. Before giving results on $\boldsymbol{P}(\bar{S}=0)$, however, we first discuss some consequences of Theorem 2.1. Let us define for $\alpha, \beta \geq 0$,

$$
\begin{aligned}
\boldsymbol{D}_{--}(\alpha, \beta):= & \beta \boldsymbol{I}_{--}-\operatorname{diag}\left(\lambda^{\alpha}\right)\left[\boldsymbol{I}_{--}-\operatorname{diag}\left(\mu^{\alpha}\right) \boldsymbol{P}_{--}^{J}\right. \\
& \left.-\operatorname{diag}\left(\mu^{\alpha}\right) \boldsymbol{P}_{-+}^{J}\left(\boldsymbol{I}_{++}-\boldsymbol{F}_{++}(\alpha, i \beta)\right)^{-1} \boldsymbol{F}_{+-}(\alpha, i \beta)\right] .
\end{aligned}
$$

It is instructive to derive the following result with the above interpretation of Theorem 2.1: consider the discrete-time process only at --points.

Corollary 2.3 For $\alpha, \beta \geq 0$ with $\boldsymbol{D}_{--}(\alpha, \beta)$ nonsingular, we have

$$
\begin{aligned}
\boldsymbol{E}_{-}\left[e^{-\alpha \bar{T}-\beta \bar{S}} ; \bar{J} \in-\right] \\
=\boldsymbol{D}_{--}(\alpha, \beta)^{-1}\left[\beta \boldsymbol{I}_{--}-\operatorname{diag}\left(\lambda^{\alpha}\right)\left(\boldsymbol{I}_{--}-\operatorname{diag}\left(\mu^{\alpha}\right) \boldsymbol{P}_{-+}^{J}\right.\right. \\
\left.\left.\times\left(\boldsymbol{I}_{++}-\boldsymbol{F}_{++}(\alpha, i \beta)\right)^{-1} \boldsymbol{F}_{+-}(\alpha, i \infty)-{ }^{\#} \widehat{\boldsymbol{P}}_{--}^{\alpha}\right)\right] \operatorname{diag}\left(\boldsymbol{P}_{-}(\bar{S}=0)\right) .
\end{aligned}
$$


Proof The claim (with characteristic functions instead of Laplace transforms) follows from $(\boldsymbol{I}-\boldsymbol{F}(\alpha, \beta))_{-+}^{-1}=(\boldsymbol{I}-\boldsymbol{F}(\alpha, \beta))_{--}^{-1} \boldsymbol{F}_{-+}(\alpha, \beta)\left(\boldsymbol{I}_{++}-\boldsymbol{F}(\alpha, \beta)\right)^{-1}$ and some elementary linear algebra. Since all characteristic functions involved are well-defined when $\beta$ is replaced by $i \beta$, we obtain the claim.

If $\boldsymbol{P}_{++}^{J}=\mathbf{0}_{++}$, using the second matrix equation of Proposition 2.1, the claim in this corollary can be reformulated in the following interesting form:

$$
\boldsymbol{E}_{-}\left[e^{-\alpha \bar{T}-\beta \bar{S}} ; \bar{J} \in-\right]=\boldsymbol{D}_{--}(\alpha, \beta)^{-1}\left[\beta \boldsymbol{I}_{--}+\boldsymbol{K}_{--}^{\alpha}\right] \operatorname{diag}\left(\boldsymbol{P}_{-}(\bar{S}=0)\right) .
$$

Our next aim is to find $\boldsymbol{P}(\bar{S}=0)$. The following lemma gives two matrix equations that must be satisfied by $\boldsymbol{P}(\bar{S}=0)$.

Lemma 2.2 $\boldsymbol{P}(\bar{S}=0)$ satisfies the system

$$
\begin{aligned}
& \boldsymbol{P}_{+}(\bar{S}=0)=\boldsymbol{F}_{++}(0, i \infty) \boldsymbol{P}_{+}(\bar{S}=0)+\boldsymbol{F}_{+-}(0, i \infty) \boldsymbol{P}_{-}(\bar{S}=0), \\
& \boldsymbol{P}_{-}(\bar{S}=0)={ }^{\#} \widehat{\boldsymbol{P}}_{-+}^{0} \boldsymbol{P}_{+}(\bar{S}=0)+{ }^{\#} \widehat{\boldsymbol{P}}_{--}^{0} \boldsymbol{P}_{-}(\bar{S}=0) .
\end{aligned}
$$

Proof The claim follows upon right-multiplication of the statement in Theorem 2.1 by 1 and choosing $\alpha=\beta=0$.

The two equations in the lemma can be described as follows. The first equation considers $\boldsymbol{P}_{+}(\bar{S}=0)$ by conditioning on the first step $\left(S_{1}, J_{1}\right)$ and using the Markov property; $J_{1}$ can both be a +-point or a --point, but $S_{1}$ cannot strictly increase. The interpretation of the second equation is slightly more complicated, and follows from arguments reminiscent of the interpretation of Theorem 2.1. Again, the idea is to partition with respect to the last-passage epoch $\ell:=\inf \left\{n: S_{n}=\sup _{m \geq n} S_{m}\right\}$, which is either a +-point or a --point. On the event $\{\bar{S}=0\}$, starting from $\left(\ell, S_{\ell}\right)$ and looking backward in time, zero is a first descending ladder epoch. On the other hand, looking forward in time from $\left(\ell, S_{\ell}\right)$, the process cannot have a strict ascending ladder epoch. Note that $\ell$ fails to be a stopping time.

We briefly pause our analysis of $\boldsymbol{P}(\bar{S}=0)$ to record the following PollaczekKhinchine type formula for $\bar{S}$.

Corollary 2.4 For $\beta>0$ with $\boldsymbol{D}_{--}(0, \beta)$ nonsingular, we have

$$
\boldsymbol{E}_{-} e^{-\beta \bar{S}}=\beta \boldsymbol{D}_{--}(0, \beta)^{-1} \boldsymbol{P}_{-}(\bar{S}=0) .
$$

Proof The corollary is a consequence of Corollary 2.3 and Lemma 2.2.

We now investigate to what extend the system of equations in Lemma 2.2 determines $\boldsymbol{P}(\bar{S}=0)$. First, since $\boldsymbol{I}_{++}-\boldsymbol{F}_{++}(0, i \infty)$ is always nonsingular by assumption, the first formula shows that it suffices to find $\boldsymbol{P}_{-}(\bar{S}=0)$ instead of the larger vector $\boldsymbol{P}(\bar{S}=0)$. Unfortunately, the whole system of equations in Lemma 2.2 is always singular. More precisely, using Eq. 3 and Proposition 2.1, we readily obtain that

$$
\boldsymbol{K}_{--}^{0} \boldsymbol{P}_{-}(\bar{S}=0)=\mathbf{0}_{-} .
$$


The following proposition shows that this determines $\boldsymbol{P}_{-}(\bar{S}=0)$ (and therefore $\boldsymbol{P}(\bar{S}=0))$ up to a constant.

Proposition 2.4 The matrix $\boldsymbol{K}_{--}^{0}$ has the following properties:

1. zero is a simple eigenvalue of $\boldsymbol{K}_{--}^{0}$, and the other $N_{-}-1$ eigenvalues have strictly negative real parts, and

2. if $N_{-}>1$, then $\operatorname{diag}\left(\lambda^{0}\right)^{-1} \boldsymbol{\pi}_{J}(-)$ and $\boldsymbol{P}_{-}(\bar{S}=0)$ are left and right eigenvectors of $\boldsymbol{K}_{--}^{0}$ respectively, corresponding to the eigenvalue zero.

Proof For the first property, it suffices to consider the matrix $\widehat{\boldsymbol{Q}}_{--}^{0}$, which is similar to $\boldsymbol{K}_{--}^{0}$. The matrix $\widehat{\boldsymbol{Q}}_{--}^{0}$ inherits its irreducibility from $\boldsymbol{P}^{J}$, and since it is an intensity matrix of a (nondefective) Markov process, the assertion follows from standard Perron-Frobenius theory.

The 'right eigenvector' part of the second claim follows from Eq. 7, and the 'left eigenvector' part translates to $\widehat{\boldsymbol{Q}}_{--}^{0} \mathbf{1}_{-}=\mathbf{0}_{-}$.

Proposition 2.4 shows that one more equation is needed to fully specify $\boldsymbol{P}_{-}(\bar{S}=0)$, and this equation is given in the following lemma. Let $\boldsymbol{\pi}_{-}$be the unique $\mathcal{I}_{-}$-probability vector satisfying

$$
\boldsymbol{\pi}_{-}^{\prime} \operatorname{diag}\left(\lambda^{0}\right)\left(\boldsymbol{P}_{--}^{J}+\boldsymbol{P}_{-+}^{J}\left(\boldsymbol{I}_{++}-\boldsymbol{P}_{++}^{J}\right)^{-1} \boldsymbol{P}_{+-}^{J}\right)=\boldsymbol{\pi}_{-}^{\prime} \operatorname{diag}\left(\lambda^{0}\right)
$$

in fact, $\boldsymbol{\pi}_{-}$is proportional to $\operatorname{diag}\left(\lambda^{0}\right)^{-1} \pi_{J}(-)$.

Lemma 2.3 We have

$$
\boldsymbol{\pi}_{-}^{\prime} \boldsymbol{P}_{-}(\bar{S}=0)=1-\boldsymbol{\pi}_{-}^{\prime} \operatorname{diag}\left(\lambda^{0}\right) \boldsymbol{P}_{-+}^{J}\left(\boldsymbol{I}_{++}-\boldsymbol{P}_{++}^{J}\right)^{-1} \boldsymbol{E}_{+} S_{1} .
$$

This equation is independent of the $N_{-}-1$ independent linear equations stemming from Eq. 7.

Proof The idea is to premultiply the expression for $\boldsymbol{P}_{-}(\bar{S}=0)$ in Corollary 2.4 by $\boldsymbol{\pi}_{-}^{\prime}$, to divide both sides by $\beta$, and then let $\beta \rightarrow 0$. By definition of $\boldsymbol{\pi}_{-}$, this immediately yields that $\boldsymbol{\pi}_{-}^{\prime} \boldsymbol{P}_{-}(\bar{S}>0)$ equals

$$
\begin{aligned}
\lim _{\beta \rightarrow 0} \frac{1}{\beta} \pi_{-}^{\prime} \operatorname{diag}\left(\lambda^{0}\right) \boldsymbol{P}_{-+}^{J}[ & \left(\boldsymbol{I}_{++}-\boldsymbol{P}_{++}^{J}\right)^{-1} \boldsymbol{P}_{+-}^{J} \\
& \left.-\left(\boldsymbol{I}_{++}-\boldsymbol{F}_{++}(0, i \beta)\right)^{-1} \boldsymbol{F}_{+-}(0, i \beta)\right] \boldsymbol{E}_{-} e^{-\beta \bar{S}} .
\end{aligned}
$$

It is not hard to see that this equals $\boldsymbol{\pi}_{-}^{\prime} \operatorname{diag}\left(\lambda^{0}\right) \boldsymbol{P}_{-+}^{J} \boldsymbol{E}_{+} S_{\gamma_{-}}$, where $\gamma_{-}:=\inf \{n \geq 1$ : $J_{n} \in \mathcal{I}_{-}$. To compute $\boldsymbol{E}_{+} S_{\gamma_{-}}$, we condition on the first step to see that the first claim follows:

$$
\boldsymbol{E}_{+} S_{\gamma_{-}}=\boldsymbol{E}_{+} S_{1}+\boldsymbol{P}_{++}^{J} \boldsymbol{E}_{+} S_{\gamma_{-}}
$$


The independence of the other $N_{-}-1$ equations is a consequence of the fact that

$$
\boldsymbol{\pi}_{-}^{\prime} \operatorname{diag}\left(\lambda^{0}\right) \boldsymbol{P}_{-+}^{J}\left(\boldsymbol{I}_{++}-\boldsymbol{P}_{++}^{J}\right)^{-1} \boldsymbol{E}_{+} S_{1}<1,
$$

due to the stability constraint $\boldsymbol{\pi}_{J}^{\prime} \boldsymbol{E} S_{1}<0$.

\subsection{The Distribution of $(\underline{S}, \underline{T}, \underline{J})$}

In this subsection, we suppose that $\boldsymbol{\pi}_{J}^{\prime} \boldsymbol{E} S_{1}>0$, so that $S$ drifts to $+\infty$. We are interested in the minimum of $S$ and related quantities.

To interpret the result, it is important to note that the matrix $\beta \boldsymbol{I}_{--}-\boldsymbol{Q}_{--}^{\alpha}$ is always nonsingular for $\beta \geq 0$, since $\boldsymbol{Q}_{--}^{\alpha}$ is a defective intensity matrix.

Theorem 2.2 For $\alpha, \beta \geq 0$, we have $\underline{J} \in \mathcal{I}_{+}$and

$$
\begin{aligned}
\boldsymbol{E}\left[e^{-\alpha \underline{T}+\beta \underline{S}} ; \underline{J} \in+\right]= & {\left[\left(\begin{array}{c}
\boldsymbol{I}_{++} \\
\mathbf{0}_{-+}
\end{array}\right)+\left(\begin{array}{c}
\boldsymbol{P}_{+\circlearrowleft-}^{\alpha} \\
\boldsymbol{I}_{--}
\end{array}\right)\left(\beta \boldsymbol{I}_{--}-\boldsymbol{Q}_{--}^{\alpha}\right)^{-1} \operatorname{diag}\left(\mu^{\alpha} \lambda^{\alpha}\right) \boldsymbol{P}_{-+}^{J}\right] } \\
& \times \operatorname{diag}\left(\mathbf{1}_{+}-\boldsymbol{P}_{+{ }^{-}}^{0} \mathbf{1}_{-}\right) .
\end{aligned}
$$

In particular, for $j \in \mathcal{I}$ and $k \in \mathcal{I}_{+}$, we have the matrix-exponential form

$$
\mathbb{P}_{j}(\underline{S}<x ; \underline{J}=k)=\left(1-\boldsymbol{e}_{k}^{\prime} \boldsymbol{P}_{+{ }_{-}-\mathbf{1}_{-}}^{0}\right) \boldsymbol{e}_{j}^{\prime}\left(\begin{array}{c}
\boldsymbol{P}_{+{ }^{-}}^{0} \\
\boldsymbol{I}_{--}
\end{array}\right) e^{-\boldsymbol{Q}_{--}^{0} x} \operatorname{diag}\left(\lambda^{0}\right) \boldsymbol{P}_{-+}^{J} \boldsymbol{e}_{k},
$$

where $x \leq 0$.

Proof The Markov property shows that for $\alpha, \beta \geq 0$,

$$
\boldsymbol{E}_{+}\left[e^{-\alpha \underline{T}+\beta \underline{S}} ; \underline{J} \in+\right]=\boldsymbol{P}_{+\circlearrowleft_{-}^{-}}^{\alpha} \boldsymbol{E}_{-}\left[e^{-\alpha \underline{T}+\beta \underline{S}} ; \underline{J} \in+\right]+\operatorname{diag}\left(\boldsymbol{P}_{+}(\underline{S}=0)\right)
$$

and

$$
\begin{aligned}
\boldsymbol{E}_{-}\left[e^{-\alpha \underline{T}+\beta \underline{S}} ; \underline{J} \in+\right]= & \operatorname{diag}\left(\frac{\mu^{\alpha} \lambda^{\alpha}}{\lambda^{\alpha}+\beta}\right) \boldsymbol{P}_{-+}^{J} \boldsymbol{E}_{+}\left[e^{-\alpha \underline{T}+\beta \underline{S}} ; \underline{J} \in+\right] \\
& +\operatorname{diag}\left(\frac{\mu^{\alpha} \lambda^{\alpha}}{\lambda^{\alpha}+\beta}\right) \boldsymbol{P}_{--}^{J} \boldsymbol{E}_{-}\left[e^{-\alpha \underline{T}+\beta \underline{S}} ; \underline{J} \in+\right] .
\end{aligned}
$$

Substitution of the first equation in the second yields, with the expression for $\boldsymbol{Q}_{--}^{\alpha}$ in Proposition 2.3,

$$
\boldsymbol{E}_{-}\left[e^{-\alpha \underline{T}+\beta \underline{S}} ; \underline{J} \in+\right]=\left(\beta \boldsymbol{I}_{--}-\boldsymbol{Q}_{--}^{\alpha}\right)^{-1} \operatorname{diag}\left(\mu^{\alpha} \lambda^{\alpha}\right) \boldsymbol{P}_{-+}^{J} \operatorname{diag}\left(\boldsymbol{P}_{+}(\underline{S}=0)\right) .
$$

The proof is finished after observing that $\boldsymbol{P}_{+}(\underline{S}=0)=\mathbf{1}_{+}-\boldsymbol{P}_{+\circlearrowleft-}^{0} \mathbf{1}_{-}$. Note that this vector is nonzero as a result of the drift condition.

\section{Markov-additive Processes and their Extremes}

In this section, we study the extremes of a continuous-time Markov-additive process $X$ with nonnegative jumps and finitely many background states. Loosely speaking, such a process is characterized by a number of Lévy processes (with nonnegative jumps) $Z^{1}, \ldots, Z^{N}$ and a continuous-time Markov process with state space 
$\{1, \ldots, N\} ; X$ behaves as $Z^{j}$ when the Markov process is in state $j$. Our goal is to find the Laplace transform of the maximum and minimum of $X$, jointly with the epoch at which they are attained and the state of the Markov process at that moment.

We first give a precise definition of the process under study (Section 3.1). Section 3.2 introduces an embedded process that falls in the framework of Section 2, so that the maximum of the embedded process equals the maximum $\bar{X}$ of the original process. This embedding facilitates the computation of the desired transform, see Section 3.3. For the minimum, a similar procedure can be followed; the analysis of $\underline{X}$ may be found in Section 3.4.

\subsection{Definitions and Assumptions}

A continuous-time Markov-additive process $\{(X(t), I(t)): t \geq 0\}$ is defined on some probability space $\left(\Omega^{\prime}, \mathcal{F}^{\prime}, \mathrm{P}\right)$ and has càdlàg paths with values in $(\mathbb{R},\{1, \ldots, N\})$. We only define Markov-additive processes with nonnegative jumps and a finite number of background states, but we refer to the classical papers (Arjas and Speed 1973; Çinlar 1972; Ney and Nummelin 1987) for the construction and properties of general Markov-additive processes.

Under $\mathrm{P},\{I(t): t \geq 0\}$ is a (finite-state) continuous-time Markovian background process, which stays in state $j$ for an exponentially $\left(q_{j}\right)$ distributed amount of time, and then jumps according to some transition matrix $\mathbf{P}^{I}$. We allow $I$ to jump to the same state. We assume that $I$ is irreducible, so that there is a unique stationary distribution $\pi_{I}$ (i.e., $\boldsymbol{\pi}_{I}^{\prime} \operatorname{diag}(q) \mathbf{P}^{I}=\boldsymbol{\pi}_{I}^{\prime} \operatorname{diag}(q)$ ). While $I(t)=j$, the process $X(t)$ behaves under $\mathrm{P}$ as a spectrally positive (i.e., without negative jumps) Lévy process $Z^{j}$, with Laplace exponent

$$
\begin{aligned}
\psi_{-Z^{j}}(\beta): & =\log \mathrm{E} \exp \left(-\beta Z^{j}(1)\right) \\
& =\frac{1}{2} \sigma_{j}^{2} \beta^{2}-c_{j} \beta-\int_{(0, \infty)}\left(1-e^{-\beta y}-\beta y \mathbf{1}_{(0,1)}(y)\right) \Pi_{j}(d y),
\end{aligned}
$$

where the Lévy measure $\Pi_{j}$ is such that $\int_{(0, \infty)}\left(1 \wedge y^{2}\right) \Pi_{j}(d y)<\infty$, and also $c_{j} \in \mathbb{R}$ and $\beta, \sigma_{j} \geq 0$. In particular, $X(0)=0$. The reason for writing $\psi_{-Z^{j}}$ instead of $\psi_{Z^{j}}$ is that we try to follow the notation of Bertoin (1996, Ch. VII) as closely as possible. Let $\psi_{-Z}(\beta)$ be the vector with elements $\psi_{-Z^{j}}(\beta), j=1, \ldots, N$.

We need some further notation related to $\psi_{-Z^{j}}$, where $j$ is such that the sample paths of $Z^{j}$ are not monotone. Then we have $\psi_{-Z^{j}}(\beta) \rightarrow \infty$ as $\beta \rightarrow \infty$. Moreover, by Hölder's inequality, $\psi_{-Z^{j}}$ is strictly convex. Let $\Phi_{-Z^{j}}(0)$ be the largest solution of the equation $\psi_{-Z^{j}}(\beta)=0$, and define $\Phi_{-Z^{j}}$ (the 'inverse' of $\psi_{-Z^{j}}$ ) as the unique increasing function $\Phi_{-Z^{j}}:[0, \infty) \rightarrow\left[\Phi_{-Z^{j}}(0), \infty\right)$ such that $\psi_{-Z^{j}}\left(\Phi_{-Z^{j}}(\beta)\right)=\beta$ for $\beta>0$.

When the background process $I$ jumps from $j$ to $k$, the process $X$ jumps according to some distribution $H_{j k}$ on $[0, \infty)$. The matrix of the Laplace transforms corresponding to these 'environmental jumps' is written as $\mathbf{H}$, i.e., element $(j, k)$ of the matrix $\mathbf{H}(\beta)$ equals $\int_{[0, \infty)} e^{-\beta x} H_{j k}(d x)$.

In the spirit of Section 2.2, we use the matrix notation

$$
\mathbf{E}\left[e^{-\beta X(t)} ; I(t)\right]:=\left\{\mathbf{E}_{j}\left[e^{-\beta X(t)} ; I(t)=k\right]: j, k=1, \ldots, N\right\},
$$


and similarly for other quantities than $X(t)$. We draw attention on the difference between $\mathbf{E}$, the matrix version of the 'continuous-time' mean $\mathrm{E}$ corresponding to $\mathrm{P}$, and $\boldsymbol{E}$, the matrix version of the 'discrete-time' mean $\mathbb{E}$ corresponding to $\mathbb{P}$.

Using this matrix notation, the definition of $(X, I)$ entails that $\mathbf{E}\left[e^{-\beta X(t)} ; I(t)\right]$ is given by $e^{t \psi_{-X}(\beta)}$, where

$$
\boldsymbol{\psi}_{-X}(\beta)=\operatorname{diag}\left(\psi_{-Z}(\beta)\right)-\operatorname{diag}(q)\left(\boldsymbol{I}-\mathbf{P}^{I} \circ \mathbf{H}(\beta)\right),
$$

with $\circ$ denoting componentwise (Hadamard) matrix multiplication. Note that for instance Asmussen (2003) uses a slightly different (yet equivalent) representation, but ours is more convenient in the context of this paper. The representation in Eq. 9 can be proven along the lines of the proof of Proposition XI.2.2 in Asmussen (2003), by setting up a differential equation for $\mathrm{E}_{j}\left[e^{-\beta X(t)} ; I(t)=k\right]$.

Each of the states $j=1, \ldots, N$ can be classified as follows. If $\sigma_{j}=0$ and $c_{j} \geq 0$, we call $j$ a subordinator state. Special cases are zero-drift states $\left(\sigma_{j}=c_{j}=0\right.$ and $\left.\Pi_{j} \equiv 0\right)$, compound Poisson states $\left(\sigma_{j}=c_{j}=0, \Pi_{j}\left(\mathbb{R}_{+}\right) \in(0, \infty)\right)$, and strict subordinator states $^{1}$ (all other subordinator states). If $\sigma_{j}=0, c_{j}<0$, and $\Pi_{j}\left(\mathbb{R}_{+}\right) \in(0, \infty)$, we call $j$ a negative-drift compound Poisson state. We say that $j$ is a negative-drift state if $\sigma_{j}=0, c_{j}<0$, and $\Pi_{j} \equiv 0$. The other states are called Brownian states; these are characterized by either $\sigma_{j}>0$ or $c_{j}<0, \Pi_{j}\left(\mathbb{R}_{+}\right)=\infty$. Therefore, if $j$ is a Brownian state, it is not necessary that $Z^{j}$ contains a Brownian component, but the terminology is convenient.

There is no one-to-one correspondence between $\psi_{-X}$ and tuples $\left(\psi_{-Z}, q, \mathbf{P}^{I}, \mathbf{H}\right)$. For instance, consider the situation that $Z^{j}$ corresponds to the sum of a Brownian motion and a compound Poisson process. Then one could equivalently do as if there are environmental jumps at the jump epochs of the Poisson process; by also adapting the transition matrix, one obtains an alternative description of the same stochastic process.

Consequently, since $I$ is allowed to make self-transitions, without loss of generality we can assume that there are neither compound Poisson states nor negative-drift compound Poisson states. Indeed, these states can be replaced by zero-drift or negative-drift states, provided the $H_{j j}$ and $q_{j}$ are changed appropriately. Throughout, we suppose that there is at least one negative-drift state or Brownian state after this simplification (if $X$ drifts to $-\infty$, then this is a consequence of the spectral positivity).

The above observations allow a partitioning of the states $1, \ldots, N$ of the background process into

(i) the strict subordinator states, labeled ' $s$ ';

(ii) the zero-drift states, labeled ' $z$ ';

(iii) the negative-drift states, labeled ' $n$ '; and

(iv) the Brownian states, labeled ' $B$ '.

In the following, we always assume that the state space $\{1, \ldots, N\}$ of $I$ is partitioned in the order $s-z-n-B$. This allows us to use block matrix notation as in Section 2.2. Sometimes, it is unnecessary to distinguish between $s$ - and $z$-states, and it is therefore convenient to refer to $s$ - and $z$-states as $\underline{s}$-states. If we use this $\underline{s}$-notation in block

\footnotetext{
${ }^{1}$ It is customary in the literature to use the term strict subordinator for a subordinator with an infinite lifetime; here, it stands for a strictly increasing subordinator.
} 
matrices, we suppose that the order is $s-z$. Similarly, we refer to $n$ - and $B$-states as $\sim$-states, again preserving the order.

We also need another probability measure on $\left(\Omega^{\prime}, \mathcal{F}^{\prime}\right)$, denoted by $\widehat{P}$. Under $\widehat{P}$, $(X, I)$ is a Markov-additive process with Laplace exponent

$$
\widehat{\boldsymbol{\psi}}_{-X}(\beta):=\operatorname{diag}\left(\boldsymbol{\pi}_{I}\right)^{-1} \boldsymbol{\psi}_{-X}^{\prime}(\beta) \operatorname{diag}\left(\boldsymbol{\pi}_{I}\right) .
$$

That is, working with $(X, I)$ under $\widehat{\mathrm{P}}$ amounts to working with the time-reversed Markov-additive process under the measure $P$, and vice versa.

We define

$$
\begin{aligned}
\bar{X}(t) & :=\sup \{X(s): 0 \leq s \leq t\}, \\
\bar{F}^{X}(t) & :=\inf \{s<t: X(s)=\bar{X}(t) \text { or } X(s-)=\bar{X}(t)\}, \\
\underline{X}(t) & :=\inf \{X(s): 0 \leq s \leq t\}, \\
\underline{F}^{X}(t) & :=\inf \{s<t: X(s)=\underline{X}(t) \text { or } X(s-)=\underline{X}(t)\} .
\end{aligned}
$$

We also set $\bar{I}(t):=I\left(\bar{F}^{X}(t)\right)$ and $\underline{I}(t)=\lim _{s \uparrow F^{X}(t)} I(s)$. It is our aim to study these quantities as $t \rightarrow \infty$, in which case we omit the time index. We study the joint Pdistributions of $\left(\bar{X}, \bar{F}^{X}, \bar{I}\right)$ (in Section 3.3) and $\left(\underline{X}, \underline{F}^{X}, \underline{I}\right.$ ) (in Section 3.4). We rely extensively on two fundamental properties of Lévy processes, which we recall in the next subsection.

\subsection{Intermezzo on Lévy Processes}

In this intermezzo, we consider a Lévy process $Z$ (i.e., there is no background process) with killing at an exponentially distributed epoch. We let $e_{q}$ denote the killing epoch with mean $1 / q$, and suppose that it is independent of $Z$. We also suppose that the process does not have negative jumps, that its paths are not monotone, and that it is not a compound Poisson process. Note that, in the terminology of the previous subsection, Lévy processes arising from 'Brownian states' satisfy this property. Moreover, the inverse $\Phi_{-Z}$ of the Laplace exponent is then well-defined.

We start with two observations that actually hold in greater generality, see for instance (Bertoin 1996, Ch. VI). The quantities $\bar{Z}, \bar{F}^{Z}, \underline{Z}$, and $\underline{F}^{Z}$ are defined similarly as for $X$. First, we have the interesting identities: for $\alpha, \beta \geq 0$,

$$
\begin{gathered}
\mathrm{E} e^{-\alpha \underline{F}^{Z}\left(e_{q}\right)+\beta \underline{Z}\left(e_{q}\right)}=\mathrm{E} e^{-\alpha \underline{F}^{Z}\left(e_{q}\right)} \mathrm{E} e^{-\beta \underline{Z}\left(e_{q+\alpha}\right)}, \\
\mathrm{E} e^{-\alpha\left(e_{q}-\bar{F}^{Z}\left(e_{q}\right)\right)-\beta\left(\bar{Z}\left(e_{q}\right)-Z\left(e_{q}\right)\right)}=\mathrm{E} e^{-\alpha\left(e_{q}-\bar{F}^{Z}\left(e_{q}\right)\right)} \mathrm{E} e^{-\beta\left(\bar{Z}\left(e_{q+\alpha}\right)-Z\left(e_{q+\alpha}\right)\right)},
\end{gathered}
$$

which can be deduced from Eq. VI.1 in conjunction with Lemma II.2 and Proposition VI.4 of Bertoin (1996).

Moreover, due to Theorem VI.5(i) of Bertoin (1996), there are two ways of decomposing $\left(e_{q}, Z\left(e_{q}\right)\right)$ into two independent vectors:

1. a vector $(\sigma, U):=\left(\bar{F}^{Z}\left(e_{q}\right), \bar{Z}\left(e_{q}\right)\right)$ related to the process till time $\bar{F}^{Z}\left(e_{q}\right)$, and

- an independent second vector $(\tau,-D):=\left(e_{q}-\bar{F}^{Z}\left(e_{q}\right), Z\left(e_{q}\right)-\bar{Z}\left(e_{q}\right)\right)$ related to the process between $\bar{F}^{Z}\left(e_{q}\right)$ and $e_{q}$. 
2. - a vector $\left(\underline{F}^{Z}\left(e_{q}\right), \underline{Z}\left(e_{q}\right)\right)$ related to the process till time $\underline{F}^{Z}\left(e_{q}\right)$ (this vector has the same distribution as $(\tau,-D))$, and

- an independent second vector $\left(e_{q}-\underline{F}^{Z}\left(e_{q}\right), Z\left(e_{q}\right)-\underline{Z}\left(e_{q}\right)\right)$ related to the process between time $\underline{F}^{Z}\left(e_{q}\right)$ and $e_{q}$ (this vector has the same distribution as $(\sigma, U))$.

For applications of this splitting at the maximum (or minimum), we refer to Dȩbicki et al. (2007), Dieker (2006) and references therein. In the special case of no jumps, Asmussen (1995) exploits this property in the context of Markov-additive processes.

Due to the assumptions that $Z$ is spectrally positive and that its paths are not monotone, $\bar{Z}\left(e_{q+\alpha}\right)-Z\left(e_{q+\alpha}\right)$ has an exponential distribution; see Theorem VII.4 of Bertoin (1996). In that case, the joint transforms of the 'upward' part $(\sigma, U)$ and 'downward' part $(\tau,-D)$ are known: for $\alpha, \beta \geq 0,(\alpha, \beta) \neq(0,0)$, we have

$$
\mathrm{E} e^{-\alpha \tau-\beta D}=\frac{\Phi_{-Z}(q)}{\Phi_{-Z}(q+\alpha)+\beta},
$$

and if furthermore $\beta \neq \Phi_{-Z}(q+\alpha)$,

$$
\mathrm{E} e^{-\alpha \sigma-\beta U}=\frac{q\left(\Phi_{-Z}(q+\alpha)-\beta\right)}{\Phi_{-Z}(q)\left(q+\alpha-\psi_{-Z}(\beta)\right)} .
$$

Here, $\psi_{-Z}$ is the Laplace exponent of $-Z$ as defined in the previous subsection. The crucial observation is that $(\tau, D)$ satisfies Assumption 2.1 with $\lambda^{\alpha}=\Phi_{-Z}(q+\alpha)$ and $\mu^{\alpha}=\Phi_{-Z}(q) / \Phi_{-Z}(q+\alpha)$. This property facilitates the application of the results of Section 2 in the context of continuous-time Markov-additive processes, as we demonstrate in the next subsection.

\subsection{The Distribution of $\left(\bar{X}, \bar{F}^{X}, \bar{I}\right)$}

We have collected all the necessary prerequisites to present an embedding that allows us to characterize the distribution of $(\bar{X}, \bar{F}, \bar{I})$. It is our aim to apply the analysis of Section 2 to the embedded process, and to reformulate the results in terms of the characteristics of the process $X$ as defined in Section 3.1.

Throughout this subsection, we suppose that $\pi_{I}^{\prime} \mathbf{E} X(1)<0$, but, as in Section 2, the majority of our results only requires the weaker assumption that $X$ drifts to $-\infty$ almost surely. This holds in particular for our main result, Theorem 3.1.

To find the distribution of $\left(\bar{X}, \bar{F}^{X}, \bar{I}\right)$, we do not monitor the full process $(X, I)$, but we record time and position at 'special' epochs only. For $\underline{s}$-states and $n$-states, these epochs are chosen as follows.

- The start of a sojourn time in an $\underline{s}$-state or an $n$-state gives rise to $\underline{s}$-points and $n$ points respectively. Note that, by right-continuity of the sample paths, the value of $X$ at these epochs includes the displacement due to a possible environmental jump.

- We also record the value of $X$ right before the end of the sojourn times in $\underline{s}$-states and $n$-states. The environmental jump at that epoch is now excluded.

For $B$-states, we record the value of $X$ at three epochs.

- The first is the start of a sojourn time in these states. The resulting points are called B-points. 
- The second is the epoch for which the maximum within the sojourn time is attained. These points are called A-points.

- Finally, as for the other states, we record the value right before the end of the sojourn time.

Note that we have thus constructed a discrete-time stochastic process from $X$ that still contains all information on the maximum of $X$. We call this process the embedded process. Importantly, as a result of the independence discussed in Section 3.2, the embedded process fits into the framework of Section 2 when the space-component of the embedded points is recorded in $S$ and the time-component in $T$. The embedding is illustrated in Fig. 1; in the realization of $X$, a negative-drift compound Poisson state has been replaced by a negative-drift state with environmental jumps and selftransitions. Note that some of the embedding points remain unlabeled, since we do not need to refer to these points. As an aside, we remark that the above embedding differs from an embedding recently introduced by Asmussen et al. (2004) for special Markov-additive processes.

Motivated by this embedding, we refer to $n$-points and $A$-points as --points (as from these points the process moves down), in accordance with the terminology of Section 2.2. The order is $n-A$. Observe that we always incorporate environmentaljump points into the embedded process, even if there are no jumps with probability one. The value of the process is then simply left unchanged.

Application of this labeling shows that we have

$$
\lambda^{\alpha}:=\left(\begin{array}{c}
\operatorname{vec}\left(\frac{q_{n}+\alpha}{-c_{n}}\right) \\
\operatorname{vec}\left(\Phi_{-Z}\left(q_{B}+\alpha\right)\right)
\end{array}\right), \quad \mu^{\alpha}:=\left(\begin{array}{c}
\operatorname{vec}\left(\frac{q_{n}}{q_{n}+\alpha}\right) \\
\operatorname{vec}\left(\frac{\Phi_{-Z}\left(q_{B}\right)}{\Phi_{-Z}\left(q_{B}+\alpha\right)}\right)
\end{array}\right) .
$$

The notation in Eq. 12 should be interpreted as follows. First, $q_{n}$ is the block vector of $q$ corresponding to $n$-points; similarly $c_{n}$ is the block vector of the drift vector $c$ corresponding to $n$. Then $\left(q_{n}+\alpha\right) /\left(-c_{n}\right)$ is the vector with element $j$ equal to $\left(q_{n, j}+\right.$ $\alpha) /\left(-c_{n, j}\right)$. The vector $q_{B}$ is defined analogously to $q_{n}$. With $k=1, \ldots, N$ being the index of the $j$-th $B$-state, the $j$-th element of $\Phi_{-Z}\left(q_{B}+\alpha\right)$ is $\Phi_{-Z^{k}}\left(q_{B, j}+\alpha\right)$. The notation used in the definition of $\mu^{\alpha}$ should be read in a similar fashion.

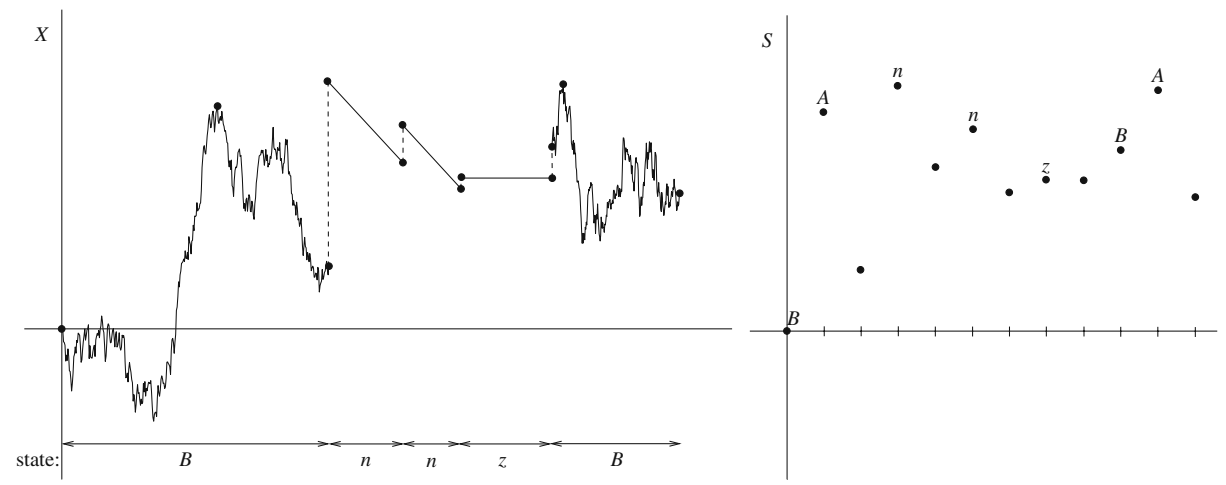

Fig. 1 The left-hand diagram represents the process $X$ with its embedding points, along with the state labels. The discrete-time embedded process $S$ is given in the right-hand diagram, along with the point labels 
It is our aim to find a characterization of $\left(\bar{X}, \bar{F}^{X}, \bar{I}\right)$ which can be regarded as the analog of Corollary 2.3. In principle, its Laplace transform can be deduced from the above embedding and the results of Section 2. However, this leads to results in terms of the embedded process as opposed to the continuous-time process $X$. It is our primary goal to obtain results in terms of $X$, and for this we need some further definitions related to displacements of the process $X$. For $\alpha, \beta \geq 0$, we set

$$
\mathbf{F}_{\underline{s} \circlearrowleft \mathcal{M}}(\alpha, \beta):=\left(\alpha \boldsymbol{I}_{\underline{s s}}-\psi_{-X \underline{s s}}(\beta)\right)^{-1} \operatorname{diag}\left(q_{\underline{s}}\right) \mathbf{P}_{\underline{s} \mathcal{M}}^{I} \circ \mathbf{H}_{\underline{s} \mathcal{M}}(\beta),
$$

where $\psi_{-X \underline{s s}}$ is the $(\underline{s}, \underline{s})$-block in the matrix $\psi_{-X}$ and ' $\mathcal{M}$ ' can be replaced by any of the blocks $s, z, n$, or $B$. The matrices $\mathbf{F}_{S_{\circlearrowleft \mathcal{M}}}(\alpha, \beta)$ and $\mathbf{F}_{z_{\circlearrowleft} \mathcal{M}}(\alpha, \beta)$ are defined similarly, with $\underline{s}$ replaced by $s$ and $z$ respectively. It is convenient to abbreviate $n$ states and $B$-states as $\sim$-states, and to impose the order $n-B$ in block matrices. Therefore, in particular, $\mathbf{F}_{\underline{S}_{\circlearrowleft}} \sim$ characterizes the displacement in time and space when we start in an $\underline{s}$-state and stay in $\underline{s}$-states until the background process jumps to a $\sim$-state. The change in the position due to the latter environmental jump is included, but the environmental jump into the first $\underline{s}$-state is not. This jump appears in the following definitions: we set for $\alpha, \beta \geq 0$,

$$
\mathbf{F}_{\uparrow \underline{s}_{\circlearrowleft} \mathcal{M}}(\alpha, \beta):=\mathbf{P}_{\sim \underline{s}}^{I} \circ \mathbf{H}_{\sim \underline{s}}(\beta) \mathbf{F}_{\underline{s}_{\circlearrowleft} \mathcal{M}}(\alpha, \beta)+\mathbf{P}_{\sim \mathcal{M}}^{I} \circ \mathbf{H}_{\sim \mathcal{M}}(\beta),
$$

where again $s, z, n$, or $B$ can be substituted for ' $\mathcal{M}$ '. The first term should be interpreted as zero if there are no $\underline{s}$-states. The measure-valued matrices $\mathbf{F}_{\uparrow \underline{s}_{\circlearrowleft} \mathcal{M}}^{\alpha}(d x)$ are defined similarly as in Section 2.3. Importantly, we have now defined $\mathbf{F}_{\uparrow \varsigma_{\circlearrowleft} \sim}(\alpha, \beta)$, which corresponds to the displacement in time and space between the end of a sojourn time in a $\sim$-state and the beginning of a sojourn time in the next $\sim$-state, including both environmental jumps.

In analogy with the discrete case, the (Markovian) last-passage process of $X$ plays a key role in our analysis. This process takes values in $\sim$-states. It follows from the analysis in Section 2 that one can associate a matrix $\boldsymbol{K}_{--}^{\alpha}$ to the embedded process. Let us define

$$
\mathcal{K}_{\sim \sim}^{\alpha}:=\operatorname{diag}\left(\frac{q \sim}{\mu^{\alpha} \lambda^{\alpha}}\right) \boldsymbol{K}_{--}^{\alpha} \operatorname{diag}\left(\frac{q \sim}{\mu^{\alpha} \lambda^{\alpha}}\right)^{-1} .
$$

The matrix $\mathcal{K}_{\sim \sim}^{\alpha}$ plays a pivotal role in the remainder. It is therefore desirable to have a representation for $\mathcal{K}_{\sim \sim}^{\alpha}$ in terms of the characteristics of $X$, much like Corollary 2.1. This is presented in the next proposition, whose proof relies on the spectral analysis of the Appendix.

Proposition 3.1 For $\alpha \geq 0$, the matrix $\mathcal{K}_{\sim \sim}^{\alpha}$ solves the nonlinear system

$$
\begin{aligned}
\alpha \boldsymbol{I}_{--}= & \left(\mathcal{K}_{\sim \sim}^{\alpha}\right)^{2} \operatorname{diag}\left(\frac{\sigma_{\sim}^{2}}{2}\right)+\mathcal{K}_{\sim \sim}^{\alpha} \operatorname{diag}\left(c_{\sim}\right) \\
& -\int_{(0, \infty)}\left(\boldsymbol{I}_{--}-e^{\mathcal{K}_{\sim \sim}^{\alpha} y}+\mathcal{K}_{\sim \sim}^{\alpha} y \mathbf{1}_{(0,1)}(y)\right) \operatorname{diag}\left(\Pi_{\sim}(d y)\right) \\
& -\operatorname{diag}(q \sim)+\int_{[0, \infty)} e^{\mathcal{K}_{\sim \sim}^{\alpha} y} \operatorname{diag}(q \sim) \mathbf{F}_{\uparrow \underline{S}_{\circlearrowleft} \sim}^{\alpha}(d y) .
\end{aligned}
$$

The solution is unique within the class of matrices with eigenvalues in the closed right complex halfplane. 
Proof Construct a 'censored embedded' process by monitoring the above discretetime embedded process only on --points and the points immediately thereafter (from which there is a nonnegative jump). In the notation of Section 2, we then have $N_{+}=N_{-}, \boldsymbol{F}_{++}(\alpha, \beta)=\mathbf{0}_{++}$, and $\boldsymbol{F}_{--}(\alpha, \beta)=\mathbf{0}_{--}$, while

$$
\begin{aligned}
& \boldsymbol{F}_{+-}(\alpha, \beta)=\mathbf{F}_{\uparrow \varsigma_{\circlearrowleft} \sim}(\alpha,-i \beta) \operatorname{diag}\left(\mathbf{E}_{\sim} e^{-\alpha \bar{F}^{Z}\left(e_{q}\right)+i \beta \bar{Z}\left(e_{q}\right)}\right), \\
& \boldsymbol{F}_{-+}(\alpha, \beta)=\operatorname{diag}\left(\frac{\mu^{\alpha} \lambda^{\alpha}}{\lambda^{\alpha}+i \beta}\right) .
\end{aligned}
$$

Using the fact that $\beta \boldsymbol{I}_{--}-\operatorname{diag}\left(\lambda^{\alpha}\right)$ equals

$$
\operatorname{diag}\left(\frac{\mu^{\alpha} \lambda^{\alpha}}{q \sim}\right) \times\left[\operatorname{diag}\left(\psi_{-Z^{\sim}}(\beta)\right)-\operatorname{diag}\left(q_{\sim}\right)-\alpha \boldsymbol{I}_{--}\right] \operatorname{diag}\left(\mathbf{E}_{\sim} e^{-\alpha \bar{F}^{Z}\left(e_{q}\right)-\beta \bar{Z}\left(e_{q}\right)}\right),
$$

we readily find that $\boldsymbol{D}_{--}$from Section 2.4 is given by

$$
\begin{aligned}
\boldsymbol{D}_{--}(\alpha, \beta)= & \operatorname{diag}\left(\frac{\mu^{\alpha} \lambda^{\alpha}}{q_{\sim}}\right)\left[\operatorname{diag}\left(\psi_{-Z^{\sim}}(\beta)\right)-\operatorname{diag}(q \sim)\left(\boldsymbol{I}_{--}-\mathbf{F}_{\uparrow \underline{s}_{\circlearrowleft} \sim}(\alpha, \beta)\right)-\alpha \boldsymbol{I}_{--}\right] \\
& \times \operatorname{diag}\left(\mathbf{E}_{\sim} e^{-\alpha \bar{F}^{Z}\left(e_{q}\right)-\beta \bar{Z}\left(e_{q}\right)}\right) .
\end{aligned}
$$

The factorization identity displayed in Eq. 26 can therefore be rewritten as

$$
\begin{aligned}
& \operatorname{diag}\left(\psi_{-Z^{\sim \sim}}(\beta)\right)-\operatorname{diag}(q \sim)\left(\boldsymbol{I}_{--}-\mathbf{F}_{\uparrow \underline{s}_{\circlearrowleft}}(\alpha, \beta)\right)-\alpha \boldsymbol{I}_{--} \\
& \quad=\left[\beta \boldsymbol{I}_{--}+\mathcal{K}_{\sim \sim}^{\alpha}\right] \boldsymbol{M}_{--}^{\prime}(\alpha, \beta),
\end{aligned}
$$

for some matrix $\boldsymbol{M}_{--}^{\prime}(\alpha, \beta)$ which is nonsingular if $\mathfrak{R}(\beta) \geq 0$. This factorization identity is the basis of the 'spectral' approach advanced in the Appendix. Using Eq. 15, the reasoning in the Appendix can be repeated verbatim to characterize $\mathcal{K}_{\sim \sim}^{\alpha}$ as the solution to the stated nonlinear system. In fact, the claim follows from Corollary 8.1 and its proof.

If one recalls the representation of the Laplace exponent of $X$ in Eq. 9, the above nonlinear system can be regarded as a matrix version of the equation $\alpha=\psi_{-Z}\left(\Phi_{-Z}(\alpha)\right)$. A spectral analysis reveals the connection with this fixed-point equation, as detailed in the Appendix. In fact, the appendix outlines how a spectral analysis can also be used to find $\mathcal{K}_{\sim \sim}^{\alpha}$ numerically, thereby complementing the discussion in Section 4 of Asmussen and Kella (2000).

Compared to Section 2, it is somewhat more involved to work with last-passage matrices in the general Markov-additive setting, due to the presence of subordinator states and Brownian states. Therefore, to formulate our next result, we set

$$
\mathbf{K}_{-z}^{\alpha}:=\int_{(0, \infty)} e^{\mathcal{K}_{\sim \sim}^{\alpha} x} \operatorname{diag}\left(q_{\sim}\right) \mathbf{F}_{\uparrow s_{\circlearrowleft z}}^{\alpha}(d x)
$$

and

$$
\mathbf{K}_{--}^{\alpha}:=\mathcal{K}_{\sim \sim}^{\alpha}-\left(\mathbf{K}_{-z}^{\alpha} \mathbf{F}_{z_{\circlearrowleft n}}(\alpha, \infty) \operatorname{diag}\left(-c_{n}\right)^{-1} \mathbf{0}_{-B}\right) .
$$


In these definitions, we use the subindices ' $-z$ ' and ' -- ' to indicate matrix dimensions, and not to refer to an embedding. We also define the $\alpha$-independent matrices

$$
\mathbf{K}_{z z}:=-\operatorname{diag}\left(q_{z}\right)\left[\boldsymbol{I}_{z z}-\mathbf{P}_{z z}^{I} \circ \mathbf{H}_{z z}(\infty)\right], \quad \mathbf{K}_{z n}:=\operatorname{diag}\left(q_{z}\right) \mathbf{P}_{z n}^{I} \circ \mathbf{H}_{z n}(\infty) \operatorname{diag}\left(-c_{n}\right)^{-1},
$$

and $\mathbf{K}_{z A}:=\mathbf{0}_{z B}$. We remark that these matrices cannot be interpreted as intensity matrices related to the last-passage process.

The following theorem is the main result of this subsection. It is the matrix version of Eq. 1, and should be compared with Eq. 6. The presence of the matrix $\psi_{-X}(\beta)-$ $\alpha \boldsymbol{I}$ is anticipated in view of the Wiener-Hopf factorization for general continuoustime Markov-additive processes by Kaspi (1982, Thm. 3.28), but our assumption of nonnegative jumps allows us to obtain a more explicit result.

Theorem 3.1 For $\alpha, \beta \geq 0$ with $\left(\boldsymbol{\psi}_{-X}(\beta)-\alpha \boldsymbol{I}\right)$ nonsingular, we have

$$
\begin{aligned}
& \mathbf{E}\left[e^{-\alpha \bar{F}^{X}-\beta \bar{X}} ; \bar{I}\right] \\
& \quad=\left(\boldsymbol{\psi}_{-X}(\beta)-\alpha \boldsymbol{I}\right)^{-1}\left(\begin{array}{ccc}
\mathbf{0}_{s s} & \mathbf{0}_{s z} & \mathbf{0}_{s-} \\
\mathbf{0}_{z s} & \mathbf{K}_{z z}-\alpha \boldsymbol{I}_{z z} & \mathbf{K}_{z-} \\
\mathbf{0}_{-s} & \mathbf{K}_{-z}^{\alpha} & \beta \boldsymbol{I}_{--}+\mathbf{K}_{--}^{\alpha}
\end{array}\right) \operatorname{diag}\left(\begin{array}{c}
\mathbf{0}_{s} \\
\boldsymbol{v}_{z} \\
\boldsymbol{v}_{-}
\end{array}\right),
\end{aligned}
$$

where the vectors $\boldsymbol{v}_{z}$ and $\boldsymbol{v}_{-}$are characterized in Lemma 3.1 below.

Proof Define

$$
\boldsymbol{C}(\alpha, \beta):=\operatorname{diag}\left(\mathbf{E} e^{-\alpha e_{q}+i \beta Z\left(e_{q}\right)}\right) \mathbf{P}^{I} \circ \mathbf{H}(-i \beta),
$$

so that

$$
(\boldsymbol{I}-\boldsymbol{C}(\alpha, \beta))^{-1}=\left[\alpha \boldsymbol{I}-\boldsymbol{\psi}_{-X}(-i \beta)\right]^{-1} \operatorname{diag}\left(q+\alpha-\psi_{-Z}(-i \beta)\right) .
$$

First suppose that $\bar{I}$ is a Brownian state. We need to show that

$$
\mathbf{E}_{\mathcal{M}}\left[e^{-\alpha \bar{F}^{X}+i \beta \bar{X}} ; \bar{I} \in B\right]=\left(\boldsymbol{\psi}{ }_{-X}(-i \beta)-\alpha \boldsymbol{I}\right)_{\mathcal{M} \sim}^{-1}\left[-i \beta \boldsymbol{I}_{-B}+\mathcal{K}_{\sim B}^{\alpha}\right] \operatorname{diag}\left(\boldsymbol{v}_{B}\right)
$$

where $\boldsymbol{v}_{B}=\operatorname{vec}\left(q_{B} / \Phi_{-Z}\left(q_{B}\right)\right) \circ \boldsymbol{P}_{A}(\bar{S}=0)$ is given in terms of the embedded process, and ' $\mathcal{M}$ ' can be any of the background states. Since there is always a strictly positive jump between a $B$-point and an $A$-point, we can use Eq. 6 by considering the embedded process only on --points. This shows that the left-hand side of Eq. 17 equals

$$
\begin{aligned}
(\boldsymbol{I} & -\boldsymbol{C}(\alpha, \beta))_{\mathcal{M} \sim}^{-1} \operatorname{diag}\left(\mathbf{E}_{\sim} e^{-\alpha \bar{F}^{Z}\left(e_{q}\right)+i \beta \bar{Z}\left(e_{q}\right)}\right) \\
& \times \operatorname{diag}\left(\lambda^{\alpha}+i \beta\right)^{-1}\left[-i \beta \boldsymbol{I}_{-B}+\boldsymbol{K}_{-A}^{\alpha}\right] \operatorname{diag}\left(\boldsymbol{P}_{A}(\bar{S}=0)\right) .
\end{aligned}
$$

We stress that $\boldsymbol{K}_{-A}^{\alpha}$ refers to the embedded process. Some algebra in conjunction with Eqs. 11, 13, and 16 shows that Eq. 17 holds.

Next suppose that $\bar{I}$ is a negative-drift state. We follow the reasoning used earlier, when deriving Theorem 2.1 with Kennedy's Wiener-Hopf interpretation. The maximum $S^{*}$ of the embedded process should be in an $n$-point, after some number of steps, say $k^{*}$. After $k^{*}$, the process should never exceed $S^{*}$. We next subtract a term 
to compensate paths for which $k^{*}$ is a strict last-passage epoch, so we use $\mathbf{K}_{-n}^{\alpha}$ instead of $\mathcal{K}_{\sim n}^{\alpha}$. In analogy with the case $\bar{I} \in B$, this leads to the term

$$
\left[\alpha \boldsymbol{I}-\boldsymbol{\psi}_{-X}(\beta)\right]_{\mathcal{M} \sim}^{-1}\left[\beta \boldsymbol{I}_{-n}+\mathbf{K}_{\sim n}^{\alpha}\right] \operatorname{diag}\left(\boldsymbol{v}_{n}\right),
$$

where $\boldsymbol{v}_{n}=\operatorname{vec}\left(-c_{n}\right) \circ \boldsymbol{P}_{n}(\bar{S}=0)$. Finally, we also need to subtract the contribution of paths for which there is a $z$-point without environmental jump right before $k^{*}$. It is readily seen that this contribution is $\left[\alpha \boldsymbol{I}-\boldsymbol{\psi}_{-X}(\beta)\right]_{\mathcal{M} z}^{-1} \mathbf{K}_{z n} \operatorname{diag}\left(\boldsymbol{v}_{n}\right)$.

A similar term also plays a role when $\bar{I}$ is a zero-drift state, which we study next. Set $\boldsymbol{v}_{z}=\boldsymbol{P}_{z}(\bar{S}=0)$. The 'base' term is

$$
-\left[\alpha \boldsymbol{I}-\boldsymbol{\psi}_{-X}(\beta)\right]_{\mathcal{M} z}^{-1}\left[\alpha \boldsymbol{I}_{z z}+\operatorname{diag}\left(q_{z}\right)\right] \operatorname{diag}\left(\boldsymbol{v}_{z}\right),
$$

and the term to be subtracted to correct for $z$ points right before $k^{*}$ now becomes $\left[\boldsymbol{\psi}_{-X}(\beta)-\alpha \boldsymbol{I}\right]_{\mathcal{M} z}^{-1} \operatorname{diag}\left(q_{z}\right) \mathbf{P}_{z z}^{I} \circ \mathbf{H}_{z z}(\infty) \operatorname{diag}\left(\boldsymbol{v}_{z}\right)$. Using the definition of $\mathbf{K}_{-z}^{\alpha}$, we readily find that the term corresponding to $k^{*}$ being a strict last-passage epoch is $\left[\alpha \boldsymbol{I}-\boldsymbol{\psi}_{-X}(\beta)\right]_{\mathcal{M} \sim}^{-1} \mathbf{K}_{-z}^{\alpha} \operatorname{diag}\left(\boldsymbol{v}_{z}\right)$.

We now show that the vectors $\boldsymbol{v}_{z}$ and $\boldsymbol{v}_{-}$can be found (up to a constant) as in Section 2.4. Indeed, the following lemma casts Lemma 2.2 and Proposition 2.4 into the general Markov-additive setting.

Lemma 3.1 The vectors $\boldsymbol{v}_{z}$ and $\boldsymbol{v}_{-}$have the following properties:

1. $\boldsymbol{v}_{z}=-\mathbf{K}_{z z}^{-1} \mathbf{K}_{z-} \boldsymbol{v}_{-}$, and

2. if there is more than one $\sim$-state, then $\boldsymbol{v}_{-}$is a right eigenvector of $\mathcal{K}_{\sim \sim}^{0}$ with corresponding eigenvalue zero.

Proof Since $\boldsymbol{v}_{z}=\boldsymbol{P}_{z}(\bar{S}=0)$ and $\boldsymbol{v}_{n}=\operatorname{vec}\left(-c_{n}\right) \circ \boldsymbol{P}_{n}(\bar{S}=0)$, application of Lemma 2.2 to the discrete-time embedded process yields

$$
\boldsymbol{v}_{z}=\mathbf{P}_{z z}^{I} \circ \mathbf{H}_{z z}(\infty) \boldsymbol{v}_{z}+\mathbf{P}_{z n}^{I} \circ \mathbf{H}_{z n}(\infty) \operatorname{diag}\left(-1 / c_{n}\right) \boldsymbol{v}_{n},
$$

which is readily rewritten as $\boldsymbol{v}_{z}=-\mathbf{K}_{z z}^{-1} \mathbf{K}_{z n} \boldsymbol{v}_{n}$, and this is the first assertion.

For the second claim, we obtain $\boldsymbol{K}_{--}^{\alpha} \boldsymbol{P}_{-}(\bar{S}=0)=\mathbf{0}_{-}$by applying Proposition 2.4 to the embedded process. Then we use Eq. 13 and $\boldsymbol{v}_{-}=\operatorname{diag}\left(q_{\sim} / \lambda^{0}\right) \boldsymbol{P}_{-}(\bar{S}=0)$.

Next we formulate a result in the same spirit as Corollary 2.4, which immediately follows from Theorem 3.1 and Lemma 3.1. It is the Markov-additive version of Eq. 1 for $\alpha=0$. A closely related formula has been obtained by Asmussen and Kella (2000, Eq. 4.1), who phrase their result in terms of the reflected process and a local-time vector. The precise relationship between the two formulas is further investigated in Section 4.2.

Corollary 3.1 For $\beta>0$ with $\psi_{-X}(\beta)$ nonsingular, we have

$$
\mathbf{E} e^{-\beta \bar{X}}=\beta \boldsymbol{\psi}_{-X}(\beta)^{-1}\left(\begin{array}{c}
\mathbf{0}_{\underline{s}} \\
\boldsymbol{v}_{-}
\end{array}\right)
$$

The vector $\boldsymbol{v}_{-}$is determined by Lemma 3.1 and the next normalization lemma, which is an analog of Lemma 2.3. Note that this lemma corrects Eq. 4.2 in Asmussen and Kella (2000). 
Lemma 3.2 We have

$$
-\boldsymbol{\pi}_{I}^{\prime} \mathbf{E} X(1)=\boldsymbol{\pi}_{I}(\sim)^{\prime} \boldsymbol{v}_{-} .
$$

Proof Since $\boldsymbol{\pi}_{I}$ satisfies $\boldsymbol{\pi}_{I}^{\prime} \operatorname{diag}(q) \mathbf{P}^{I}=\boldsymbol{\pi}_{I}^{\prime} \operatorname{diag}(q)$, Corollary 3.1 shows that for $\beta>0$,

$$
\frac{1}{\beta} \boldsymbol{\pi}_{I}^{\prime}\left[\operatorname{diag}\left(\psi_{-Z}(\beta)\right)-\operatorname{diag}(q)\left(\mathbf{P}^{I}-\mathbf{P}^{I} \circ \mathbf{H}(\beta)\right)\right] \mathbf{E} e^{-\beta \bar{X}}=\boldsymbol{\pi}_{I}(\sim)^{\prime} \boldsymbol{v}_{-} .
$$

Now let $\beta \rightarrow 0$ to obtain that $-\boldsymbol{\pi}_{I}^{\prime}\left[\mathbf{E} Z(1)+\operatorname{diag}(q) \mathbf{P}^{I} \circ \int x \mathbf{H}(d x)\right]=\boldsymbol{\pi}_{I}(\sim)^{\prime} \boldsymbol{v}_{-}$. Using Corollary XI.2.9(b) and (the second equality in) Corollary XI.2.5 of Asmussen (2003), it is not hard to see that the left-hand side equals $-\boldsymbol{\pi}_{I}^{\prime} \mathbf{E} X(1)$.

\subsection{The Distribution of $\left(\underline{X}, \underline{F}^{X}, \underline{I}\right)$}

In this subsection, we study the minimum of $X$ if it drifts to $+\infty$. More specifically, we establish the analogs of Proposition 3.1 and Theorem 3.1. We suppose throughout this subsection that $\boldsymbol{\pi}_{I}^{\prime} \mathbf{E} X(1)>0$.

As before, we do not monitor the full process $(X, I)$, but we only record for $\underline{s}$ states and $n$-states the time and position at the start (leading to $\underline{s}$-points and $n$-points, respectively) and immediately before the end of the sojourn time, and for $B$-states in addition the minimum within the sojourn times (leading to $A$-points). Note that the embedding is different from the one used in the previous subsection. In fact, in view of the conventions in Section 2.2, the --points are labeled differently: $n$-points and $B$-points are now --points. Since the underlying process $X$ is the same as in the previous subsection, we continue to refer to $n$-states and $B$-states (i.e., for the process $X)$ as $\sim$-states, and we still use the same F-quantities since these do not depend on the embedding.

For fixed $\alpha \geq 0$, a matrix $\mathcal{Q}_{\sim \sim}^{\alpha}$, related to the first-passage process for the embedded process, plays a similar role as $\mathcal{K}_{\sim \sim}^{\alpha}$ in the previous subsection. The characterization of $\mathcal{Q}_{\sim \sim}^{\alpha}$ given in the next proposition is the analog of Proposition 3.1.

Proposition 3.2 For $\alpha \geq 0$, the matrix $\mathcal{Q}_{\sim \sim}^{\alpha}$ solves the nonlinear system

$$
\begin{aligned}
\alpha \boldsymbol{I}_{--}= & \operatorname{diag}\left(\frac{\sigma_{\sim}^{2}}{2}\right)\left(\mathcal{Q}_{\sim \sim}^{\alpha}\right)^{2}+\operatorname{diag}\left(c_{\sim}\right) \mathcal{Q}_{\sim \sim}^{\alpha} \\
& -\int_{(0, \infty)} \operatorname{diag}\left(\Pi_{\sim}(d y)\right)\left(\boldsymbol{I}_{--}-e^{\mathcal{Q}_{\sim \sim}^{\alpha} y}+\mathcal{Q}_{\sim \sim}^{\alpha} y \mathbf{1}_{(0,1)}(y)\right) \\
& -\operatorname{diag}(q \sim)\left[\boldsymbol{I}_{--}-\int_{[0, \infty)} \mathbf{F}_{\uparrow \underline{s}_{\circlearrowleft} \sim}^{\alpha}(d y) e^{\mathcal{Q}_{\sim \sim}^{\alpha} y}\right] .
\end{aligned}
$$

The solution is unique within the class of matrices with eigenvalues in the open right complex halfplane.

Proof The proof is similar to the proof of Proposition 3.1. Again we introduce a censored embedded process by only monitoring the embedded process on --points and the points immediately thereafter. Note that this results in a different censored embedded process than in the previous subsection, since the underlying embedded 
processes differ. In the notation of Section 2, the censored embedded process has $N_{+}=N_{-}, \boldsymbol{F}_{++}(\alpha, \beta)=\mathbf{0}_{++}$, and $\boldsymbol{F}_{--}(\alpha, \beta)=\mathbf{0}_{--}$, while for $\alpha, \beta \geq 0$,

$$
\begin{aligned}
\boldsymbol{F}_{+-}(\alpha, \beta)= & \operatorname{diag}\left(\mathbf{E}_{\sim} e^{-\alpha \bar{F}^{Z}\left(e_{q}\right)+i \beta \bar{Z}\left(e_{q}\right)}\right) \\
& \times\left[\mathbf{P}_{\sim \underline{S}}^{I} \circ \mathbf{H}_{\sim \underline{s}}(-i \beta) \mathbf{F}_{\underline{s}_{\circlearrowleft}}(\alpha,-i \beta)+\mathbf{P}_{\sim \sim}^{I} \circ \mathbf{H}_{\sim \sim}(-i \beta)\right], \\
\boldsymbol{F}_{-+}(\alpha, \beta)= & \operatorname{diag}\left(\frac{\mu^{\alpha} \lambda^{\alpha}}{\lambda^{\alpha}+i \beta}\right) .
\end{aligned}
$$

For fixed $\alpha \geq 0$, the first-passage process for the embedded process is a (defective) Markov process, and we write $\mathcal{Q}_{\sim \sim}^{\alpha}$ for its intensity matrix.

In conjunction with Eq. 14, given the current embedding, $\boldsymbol{D}_{--}$from Section 2.4 can be written as

$$
\boldsymbol{D}_{--}(\alpha, \beta)=\operatorname{diag}\left(\boldsymbol{u}_{-}\right)\left[\operatorname{diag}\left(\psi_{-Z^{\sim}}(\beta)\right)-\operatorname{diag}\left(q_{\sim}\right)\left(\boldsymbol{I}_{--}-\mathbf{F}_{\uparrow \underline{s}_{\circlearrowleft} \sim}(\alpha, \beta)\right)-\alpha \boldsymbol{I}_{--}\right],
$$

for some (known) vector $\boldsymbol{u}_{-}$. Factorization identity Eq. 27 can thus be rewritten as

$$
\operatorname{diag}\left(\psi_{-Z^{\sim}}(\beta)\right)-\operatorname{diag}\left(q_{\sim}\right)\left(\boldsymbol{I}_{--}-\mathbf{F}_{\uparrow \underline{S}_{\circlearrowleft} \sim}(\alpha, \beta)\right)-\alpha \boldsymbol{I}_{--}=\boldsymbol{N}_{--}^{\prime}(\alpha, \beta)\left[\beta \boldsymbol{I}_{--}+\mathcal{Q}_{\sim \sim}^{\alpha}\right],
$$

for some matrix $\boldsymbol{N}_{--}^{\prime}(\alpha, \beta)$ which is nonsingular if $\Re(\beta) \geq 0$. This factorization is the Markov-additive analog of Eq. 27, which is the starting point for the spectral analysis for $\boldsymbol{Q}_{--}^{\alpha}$ in the Appendix. The arguments leading to Corollary 8.1 and its proof can be repeated here.

The preceding proposition generalizes the results in Section 5.3 of Miyazawa and Takada (2002) and Proposition 2(i) of Pistorius (2006). In comparison with Proposition 3.1, we note that the place of the matrices $\mathcal{Q}_{\sim \sim}^{\alpha}$ and $\exp \left(\mathcal{Q}_{\sim \sim}^{\alpha} x\right)$ is different: instead of premultiplied, they are now postmultiplied. This is in line with the correspondence between Corollaries 2.1 and 2.2.

We need some further notation to give the Laplace transform of $\left(\underline{X}, \underline{F}^{X}, \underline{I}\right)$. We define the measure $\mathbf{F}_{s \underline{S}_{\circlearrowleft} \sim}^{\alpha}(d x)$ through its Laplace transform

$$
\mathbf{F}_{s \underline{\circlearrowleft} \sim}(\alpha, \beta):=\operatorname{diag}\left(\mathbf{E}_{s} e^{-\alpha e_{q}+i \beta Z\left(e_{q}\right)}\right)\left[\mathbf{P}_{s \underline{S}}^{I} \circ \mathbf{H}_{s \underline{s}}(-i \beta) \mathbf{F}_{\underline{S}_{\circlearrowleft} \sim}(\alpha, \beta)+\mathbf{P}_{s \sim}^{I} \circ \mathbf{H}_{s \sim}(-i \beta)\right],
$$

and set

$$
\widetilde{\mathbf{P}}_{s \sim}^{\alpha}=\int_{(0, \infty)} \mathbf{F}_{s \underline{S}_{\circlearrowleft}}^{\alpha}(d x) e^{\mathcal{Q}_{\sim \sim}^{\alpha} x}
$$

Our next result is the main result of this subsection. 
Theorem 3.2 For $\alpha, \beta \geq 0$, we have

$$
\begin{aligned}
& \mathbf{E}\left[e^{-\alpha \underline{F}^{X}+\beta \underline{X}} ; \underline{I}\right]=\operatorname{diag}\left(\begin{array}{c}
\mathbf{1}_{s}-\widetilde{\mathbf{P}}_{s \sim}^{0} \mathbf{1}_{-} \\
\mathbf{F}_{z_{\circlearrowleft} \sim}(0, \infty) \mathbf{1}_{-}-\mathbf{F}_{z_{\circlearrowleft S}}(0, \infty) \widetilde{\mathbf{P}}_{s \sim}^{0} \mathbf{1}_{-} \\
\mathbf{0}_{-}
\end{array}\right)
\end{aligned}
$$

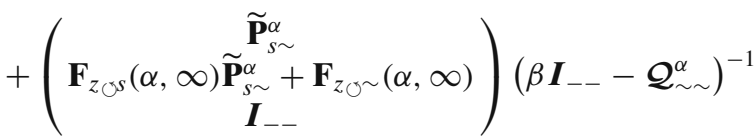

$$
\begin{aligned}
& \times \operatorname{diag}\left(\mu^{\alpha} \lambda^{\alpha}\right)\left(\mathbf{0}_{-\underline{s}}-\operatorname{diag}\left(\operatorname{diag}\left(\lambda^{0}\right)^{-1} \mathcal{Q}_{\sim \sim}^{0} \mathbf{1}_{-}\right)\right) \text {. }
\end{aligned}
$$

Proof Consider the censored embedding introduced in the proof of Proposition 3.2. It is readily seen that

$$
\mathbf{E}_{\sim}\left[e^{-\alpha \underline{F^{X}}+\beta \underline{X}} ; \underline{I} \in \sim\right]=\boldsymbol{E}_{-}\left[e^{-\alpha \underline{T}+\beta \underline{S}} ; \underline{J} \in+\right],
$$

and the latter is readily found with Theorem 2.2. The other claims follow along the lines of the proof of Theorem 2.2.

We conclude this section with a relationship between $\mathcal{Q}_{\sim \sim}^{\alpha}$ and $\widehat{\mathcal{K}}_{\sim \sim}^{\alpha}$, which can be regarded as the analog of Eq. 3. The matrix $\widehat{\mathcal{K}}_{\sim \sim}^{\alpha}$ is defined as $\mathcal{K}_{\sim \sim}^{\alpha}$, but with the dynamics of the Markov-additive process specified by the time-reversed Laplace exponent $\widehat{\psi}_{-X}$ instead of $\boldsymbol{\psi}_{-X}$. The next lemma formalizes the intuition that the lastpassage matrices under the measure $\widehat{P}$ are closely related to the first-passage matrices under the measure $P$.

Lemma 3.3 For $\alpha \geq 0$, we have

$$
\mathcal{Q}_{\sim \sim}^{\alpha}=\operatorname{diag}\left(\boldsymbol{\pi}_{I}(\sim)\right)^{-1}\left[\widehat{\mathcal{K}}_{\sim \sim}^{\alpha}\right]^{\prime} \operatorname{diag}\left(\boldsymbol{\pi}_{I}(\sim)\right) .
$$

Proof First we note that, since their proofs rely on the appendix, to apply Propositions 3.1 and 3.2 we do not need that $X$ drifts to $-\infty$ or $+\infty$, respectively. The matrix $\widehat{\mathcal{K}}_{\sim \sim}^{\alpha}$ satisfies the system given in Proposition 3.1, but with $\mathbf{F}_{\uparrow \underline{s}_{\circlearrowleft} \sim}^{\alpha}(d x)$ replaced by its time-reversed counterpart $\widehat{\mathbf{F}}_{\uparrow_{\underline{s}_{\circlearrowleft} \sim}^{\alpha}}^{\alpha}(d x)$. Using $\widehat{\mathbf{F}}_{\uparrow_{\underline{s}_{\circlearrowleft}} \sim}(\alpha, \beta)=$ $\operatorname{diag}\left(\boldsymbol{\pi}_{I}(\sim)\right)^{-1} \mathbf{F}_{\uparrow \underline{S}_{\circlearrowleft} \sim}^{\prime}(\alpha, \beta) \operatorname{diag}\left(\boldsymbol{\pi}_{I}(\sim)\right)$, the matrix on the right-hand side of Eq. 19 is seen to satisfy the same matrix equation as $\mathcal{Q}_{\sim \sim}^{\alpha}$ given in Proposition 3.2. Uniqueness of its solution proves the claim.

\section{The Fluid Queue: Theory}

In this section, we use the theory developed in the previous sections to analyze a single fluid queue. We stress that our treatment of the single fluid queue is of crucial importance for understanding the network results of Section 6. In a fluid queue, work (fluid) arrives at a storage facility, where it is gradually drained; if the input temporarily exceeds the output capacity, then work can be stored in a buffer.

More precisely, the system dynamics of the fluid queue are as follows. Let $\{(A(t), I(t)): t \geq 0\}$ be a continuous-time stochastic process, defined on some 
measurable space, such that for any $t \geq 0, A(t)$ is the amount of work offered to the system in the interval $[0, t]$ and $I(t)$ is the state of some background process at time $t$. The buffer can be interpreted as a fluid reservoir, to which input is offered according to the input process $A$. The buffer is drained at a constant rate $r$, i.e., a tap at the bottom of the fluid reservoir releases fluid at rate $r$ as long as the buffer is nonempty. After the fluid is processed, it immediately leaves the system. Throughout, we suppose that the buffer capacity is unlimited.

We write $W(t)$ for the amount of fluid in the buffer at time $t$, and call this the buffer content. The buffer-content process is also known as a (stochastic) storage process. A busy period starts when the buffer becomes nonempty (i.e., the buffer content becomes positive). The age of the busy period at time $t$, written as $B(t)$, indicates how long ago a busy period started; in a formula, this means that

$$
B(t):=t-\sup \{s \leq t: W(s)=0\} .
$$

It is our aim to study the distribution of $(W(t), B(t), I(t))$ in steady-state, i.e., as $t \rightarrow$ $\infty$, for a number of different input processes. We abbreviate $W(\infty), B(\infty)$, and $I(\infty)$ as $W, B$, and $I$ respectively; their existence follows from assumptions that we impose later on.

\subsection{Markov-modulated ON/OFF Input}

Suppose that the input process corresponds to a single source that is driven by a background process $I$ that switches between $N$ states. The transitions of the background process are governed by an irreducible Markov chain $J$, defined through the transition probability matrix $\boldsymbol{P}^{J}:=\left\{p_{j k}^{J}: j, k=1, \ldots, N\right\}$; the sojourn times in the each of the $N$ states are specified below. Suppose that $J$ and all other random objects in this subsection are defined on the probability space $(\Omega, \mathcal{F}, \mathbb{P})$.

If the background process is in state $j$ for $j=1, \ldots, N-1$, it feeds work into the reservoir at a constant rate $R_{j}<r$. Since the fluid level decreases during these periods, we call the corresponding states $O F F$-states. The lengths of the sojourn times in these states are all mutually independent. Moreover, the sojourn time in OFF-state $j$ is exponentially distributed with parameter $q_{j}$.

If the source is in state $N$, the so-called $O N$-state, the source generates work according to a generic stochastic process $\left\{A_{\mathrm{ON}}(t): t \geq 0\right\}$. In order to ensure that the buffer content does not decrease (strictly) while the source emits fluid, we suppose that $A_{\mathrm{ON}}(t) \geq r t$ for any $t \geq 0$ almost surely. The ON-period is terminated after some period distributed as the generic random variable $T^{(\mathrm{k})}>0$ ('killing time'), independent of $A_{\mathrm{ON}}$. After this ON-period, $I$ always makes a transition to an OFFstate (i.e., $J$ has no self-transitions in state $N$ ). We suppose that $\mathbb{E} T^{(\mathrm{k})}<\infty$. In principle, the probability distribution governing the transitions to OFF-states may depend on (the whole trajectory of) $A_{\mathrm{ON}}$ and $T^{(\mathrm{k})}$, but we suppose for simplicity that this is not the case. The ON-periods are mutually independent, and also independent of the OFF-periods.

To characterize the distribution of $(W, B, I)$, we use an embedding and the theory from Section 2. Let $T^{(\mathrm{k})^{*}}$ be distributed as the elapsed time that the source is ON, if 
we observe the system in steady state in an ON-state. That is, it has the integrated-tail distribution

$$
\mathbb{P}\left(T^{(\mathrm{k})^{*}}>y\right)=\frac{1}{\mathbb{E} T^{(\mathrm{k})}} \int_{y}^{\infty} \mathbb{P}\left(T^{(\mathrm{k})}>x\right) d x,
$$

where $y \geq 0$. We also need the expected sojourn time between ON-states, $\mathbb{E} V_{\mathrm{OFF}}$. Standard formulas for moments of phase-type distributions show that

$$
\mathbb{E} V_{\mathrm{OFF}}=\boldsymbol{P}_{N-}^{J}\left(\boldsymbol{I}_{--}-\boldsymbol{P}_{--}^{J}\right)^{-1} \operatorname{vec}\left(\frac{1}{q_{-}}\right),
$$

where the beginnings of the OFF-sojourn times and ON-sojourn times are labeled as --points and +-points respectively, as in Section 2 . The quantity $\mathbb{E} V_{\text {OFF }}$ plays an important role for the probability $p_{k}$ that the source is in state $k$ when the system is in steady state. We find that

$$
\begin{aligned}
p_{k} & =\frac{\mathbb{E} V_{\mathrm{OFF}}}{\mathbb{E} V_{\mathrm{OFF}}+\mathbb{E} T^{(\mathrm{k})}} \frac{\pi_{J}(k)}{\pi_{J}(-)^{\prime} \operatorname{vec}\left(q_{k} / q_{-}\right)}, k=1, \ldots, N-1 ; \\
p_{N} & =\frac{\mathbb{E} T^{(\mathrm{k})}}{\left(\mathbb{E} V_{\mathrm{OFF}}+\mathbb{E} T^{(\mathrm{k})}\right)} .
\end{aligned}
$$

The stability condition of this model is

$$
\frac{\mathbb{E} A_{\mathrm{ON}}\left(T^{(\mathrm{k})}\right)}{\mathbb{E} V_{\mathrm{OFF}}+\mathbb{E} T^{(\mathrm{k})}}+\boldsymbol{R}_{-}^{\prime} \boldsymbol{p}_{-}<r .
$$

We write $\widehat{\boldsymbol{P}}^{J}=\left\{\widehat{p}_{j k}^{J}: j, k=1, \ldots, N\right\}$ for the time-reversed transition matrix of the Markov process $J$, and we define $\widehat{\mathbb{P}}$ such that $(S, T, J)$ has the transition kernel

$$
\begin{aligned}
& \widehat{p}((s, t, j),(s+d v, t+d w, k)) \\
& \quad= \begin{cases}\widehat{p}_{j k}^{J} \mathbb{P}(U \in d v, \sigma \in d w) & j=N \text { and } k=1, \ldots, N ; \\
\widehat{p}_{j k}^{J} \mathbb{P}\left(-D^{j} \in d v, \tau^{j} \in d w\right) & j=1, \ldots, N-1 \text { and } k=1, \ldots, N,\end{cases}
\end{aligned}
$$

with

$$
\mathbb{E} e^{-\alpha \sigma-\beta U}=\mathbb{E}\left[e^{-\alpha T^{(\mathrm{k})}-\beta\left[A_{\mathrm{ON}}(\mathrm{k})-r T^{(\mathrm{k})}\right]}\right], \quad \mathbb{E} e^{-\alpha \tau^{j}-\beta D^{j}}=\frac{q_{j}}{q_{j}+\alpha+\beta\left(r-R_{j}\right)} .
$$

We next express the distribution of $(W, B, I)$ in terms of the distribution of $(S, T)$.

Proposition 4.1 For $k=1, \ldots, N-1, \omega, \beta \geq 0$, we have

$$
\mathbb{E}\left[e^{-\omega W-\beta B} ; I=k\right]=p_{k} \widehat{\mathbb{E}}_{k} e^{-\omega \bar{S}-\beta \bar{T}},
$$

and

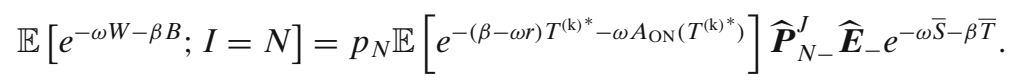

Proof The proof relies elements from regenerative-processes theory, cf. the construction used in Theorem 4 in Kella and Whitt (1992a). We here specialize to just 
$W$; the proof of the stated result, which also covers the age of the busy period $B$ as well as the state of the background process $I$, works analogously.

- The classical Reich formula states that, denoting $\mathscr{W}(t):=-\widehat{A}(-t)-r t$,

$$
W \stackrel{\mathrm{d}}{=} \sup _{t \geq 0} \mathscr{W}(t)
$$

with $\widehat{A}(-t)$ being the work generated in the interval $[-t, 0]$, where the system started in steady state at time $-\infty$. This entails that the maximum value attained by the process $\mathscr{W}(t)=-\widehat{A}(-t)-r t$ needs to be analyzed; realize that increasing $t$ corresponds to looking backward in time.

- To analyze $\sup _{t \geq 0} \mathscr{W}(t)$, the state of the background process at time zero is sampled from $\boldsymbol{p}$ (as determined in Eq. 20). Two possibilities arise: the background process is in the ON-state $N$, or in one of the OFF-states $1, \ldots, N-1$ :

$$
\mathbb{E}\left[e^{-\omega W}\right]=p_{N} \mathbb{E}_{N}\left[\exp \left(-\omega \sup _{t \geq 0} \mathscr{W}(t)\right)\right]+\sum_{k=1}^{N-1} p_{k} \mathbb{E}_{k}\left[\exp \left(-\omega \sup _{t \geq 0} \mathscr{W}(t)\right)\right]
$$

- The initial state is $N$. Using the argumentation of Kella and Whitt (1992a), it is seen that the background process stays in this state for a period that has the integrated-tail distribution of $T^{(\mathrm{k})}$; the increment of $\mathscr{W}(t)$ is distributed as $A_{\mathrm{ON}}\left(T^{(\mathrm{k})^{*}}\right)-r T^{(\mathrm{k})^{*}}$ (which is nonnegative, as $N$ is ON-state). The next state, say $j$ (which is necessarily an OFF-state), is sampled using the timereversed transition probabilities $\widehat{\boldsymbol{P}}_{N-}^{J}$. It is readily seen that the supremum of $\mathscr{W}(t)$ over $t \geq 0$ equals $A_{\mathrm{ON}}\left(T^{(\mathrm{k})^{*}}\right)-r T^{(\mathrm{k})^{*}}$ increased by

$$
\sup _{t>T^{(\mathrm{k})^{*}}}-\widehat{A}(-t)-r t+\widehat{A}\left(-T^{(\mathrm{k})^{*}}\right)+r T^{(\mathrm{k})^{*}}
$$

where the 'initial' state (that is, the state at time $-T^{(\mathrm{k})^{*}}$ ) of the background process is $j$. Then realize that Eq. 21 is distributed as $\sup _{t \geq 0} \mathscr{W}(t)$, but now started in $j$ rather than $N$.

- The initial state is $k=1, \ldots, N-1$. It stays in this initial state for a period that has the integrated-tail distribution of $\tau^{k}$, which is again exponential with parameter $q_{k}$; as a consequence we could do as if the background process had just jumped to $k$ at time zero. The supremum of $\mathscr{W}(t)$ over $t \geq 0$ can thus immediately be expressed in terms of the time-reversed embedded process.

The stated follows by combining the above findings.

Expressions for the $\widehat{\mathbb{E}}_{k} e^{-\omega \bar{S}-\beta \bar{T}}$ in Proposition 4.1 for $k=1, \ldots, N-1$ can be found with the theory of Section 2. Hence, in order to use the above theorem, it remains to find an expression for the transform of $\left(T^{(\mathrm{k})^{*}}, A_{\mathrm{ON}}\left(\mathrm{k}^{*}\right)\right)$; from Scheinhardt and Zwart (2002) we have

$$
\mathbb{E}\left[e^{-\alpha T^{(\mathrm{k})^{*}}-\beta A_{\mathrm{ON}}\left(T^{(\mathrm{k})^{*}}\right)}\right]=\frac{1}{\mathbb{E} \mathrm{k}} \mathbb{E}\left[\int_{0}^{T^{(\mathrm{k})}} e^{-\alpha t-\beta A_{\mathrm{ON}}(t)} d t\right] .
$$


When specialized to the distribution of $W$ and using Eq. 22, Proposition 4.1 reduces to

$$
\mathbb{E} e^{-\omega W}=\left(\boldsymbol{p}_{-}^{\prime}+\frac{p_{N}}{\mathbb{E} T^{(\mathrm{k})}} \mathbb{E}\left[\int_{0}^{T^{(\mathrm{k})}} e^{-\omega\left[A_{\mathrm{ON}}(t)-r t\right]} d t\right] \widehat{\boldsymbol{P}}_{N-}^{J}\right) \widehat{\boldsymbol{E}}_{-} e^{-\omega \bar{S}} .
$$

In Boxma et al. (2001), a similar expression has been interpreted as a decomposition of $W$ in terms of a clearing process and an independent dam process.

\subsection{Markov-additive Input}

In this subsection, we suppose that there is an irreducible Markov process $I$ such that $(A, I)$ is a Markov-additive process on some probability space $\left(\Omega^{\prime}, \mathcal{F}^{\prime}, \mathrm{P}\right)$. We define $X(t):=A(t)-r t$, the free process. Clearly, $(X, I)$ is a Markov-additive process as well. Even though Proposition 4.2 below holds in much greater generality, we suppose throughout that $X$ does not have negative jumps. Consequently, this subsection relies extensively on Theorem 3.1. We do not analyze the spectrally negative case, but it could be analyzed with Theorem 3.2; further details can be found in Miyazawa and Takada (2002).

In Fig. 2, we have plotted a possible realization of the process $W$. Note that in this diagram there are Brownian states, subordinator states, and negative-drift states.

We now establish the precise relationship between the buffer-content process and extremes of the free process, which follows from the reasoning in Section II.3 and Section VI.7 of Asmussen (2000); see also Section 4 of Miyazawa and Takada (2002). Again, $(B(0), W(0), I(0)$ does not have influence on the behavior of $(B(t), W(t), I(t))$ as $t \rightarrow \infty$, a property that is intuitively clear. The result follows by the same arguments as those used for Markov-modulated ON/OFF input, but no 'residual' (or 'clearing-model') quantities are needed since the sojourn times of $I$ are exponential. We write $\widehat{\mathrm{P}}_{k}$ for the law of the Markov-additive process $(X, I)$ with $I(0)=k$ and Laplace exponent $\widehat{\psi}_{-X}$ defined in Eq. 10 .

Proposition 4.2 Suppose that $\pi_{I}^{\prime} \mathrm{E} X(1)<0$. Then $(W, B)$ is a finite random vector, and for any $\omega, \beta \geq 0, k=1, \ldots, N$, we have

$$
\mathrm{E}\left[e^{-\omega W-\beta B} ; I=k\right]=\pi_{I}(k) \widehat{\mathrm{E}}_{k} e^{-\beta \bar{F}^{X}-\omega \bar{X}} .
$$

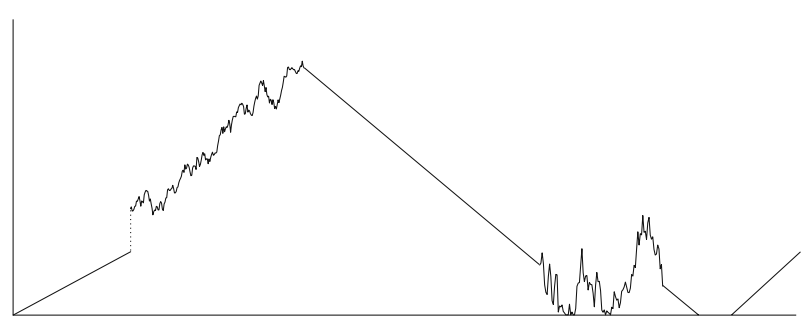

Fig. 2 A realization of $W$ 
We now work out the preceding proposition for the distribution of $(W, I)$, since the resulting formula is particularly appealing. Corollary 3.1 shows that for $\omega \geq 0$, provided $\widehat{\psi}_{-X}(\omega)$ is nonsingular,

$$
\operatorname{diag}\left(\boldsymbol{\pi}_{I}\right) \widehat{\mathbf{E}} e^{-\omega \bar{X}}=\omega \operatorname{diag}\left(\boldsymbol{\pi}_{I}\right) \widehat{\boldsymbol{\psi}}_{-X}^{-1}(\omega)\left(\begin{array}{c}
\mathbf{0}_{\underline{S}} \\
\widehat{\boldsymbol{v}}_{-}
\end{array}\right)=\omega\left[\boldsymbol{\psi}_{-X}^{\prime}(\omega)\right]^{-1}\left(\begin{array}{c}
\mathbf{0}_{\underline{\underline{s}}} \\
\boldsymbol{u}_{-}
\end{array}\right),
$$

where we set $\boldsymbol{u}_{-}:=\pi_{I}(\sim) \circ \widehat{\boldsymbol{v}}_{-}$(recall that $\sim$-states stand for $n$-states and $B$-states). The vector $\widehat{\boldsymbol{v}}_{-}$is defined in the same way as the vector $\boldsymbol{v}_{-}$-vector, but with $\mathrm{P}$ replaced by $\widehat{P}$. With Proposition 4.2, this leads immediately to the identity

$$
\mathrm{E}\left[e^{-\omega W} ; I\right]=\omega\left(\mathbf{0}_{\underline{s}}^{\prime} \boldsymbol{u}_{-}^{\prime}\right) \boldsymbol{\psi}_{-X}(\omega)^{-1}
$$

for $\omega \geq 0$ with $\psi_{-X}(\omega)$ nonsingular. This formula is Eq. 4.1 of Asmussen and Kella (2000), who interpret $\boldsymbol{u}_{-}$in terms of local times. The following observation, however, is new. By combining Lemma 3.3 with Lemma 3.1, it readily follows that $\boldsymbol{u}_{-}$must be a left eigenvector of $\mathcal{Q}_{\sim \sim}^{0}$ (corresponding to the simple eigenvalue zero); this uniquely determines $\boldsymbol{u}_{-}$up to a constant. This constant can be found by writing down the formula for $\mathrm{E} e^{-\omega W}$ from Eq. 23, using $\mathbf{1}=\mathbf{P}^{I} \mathbf{1}$, and letting $\omega \rightarrow 0$ in the resulting expression.

Motivated by Proposition 4.2, we next characterize the $\widehat{\mathrm{P}}$-distribution of $\left(\bar{X}, \bar{F}^{X}, \bar{I}\right)$ (the last component is not required here, but it is needed in Section 6). To avoid the introduction of yet more matrices, we suppose that there are no zerodrift states. The following result then follows immediately from Theorem 3.1 and Lemma 3.3.

Corollary 4.1 Suppose that $\boldsymbol{\pi}_{I}^{\prime} \mathbf{E} X(1)<0$ and that there are no zero-drift states. We then have for $\alpha, \beta \geq 0$,

$$
\left(\boldsymbol{\psi}_{-X}^{\prime}(\beta)-\alpha \boldsymbol{I}\right) \operatorname{diag}\left(\boldsymbol{\pi}_{I}\right) \widehat{\mathbf{E}}\left[e^{-\alpha \bar{F}^{X}-\beta \bar{X}} ; \bar{I}\right]=\left(\begin{array}{l}
\mathbf{0}_{s s} \\
\mathbf{0}_{-s}\left(\beta \boldsymbol{I}_{--}+\left[\mathcal{Q}_{\sim \sim}^{\alpha}\right]^{\prime}\right) \operatorname{diag}\left(\boldsymbol{u}_{-}\right)
\end{array}\right) .
$$

In conclusion, if $X$ is spectrally positive, the matrix $\mathcal{Q}_{\sim \sim}^{\alpha}$ plays a similar role for the steady-state buffer-content process as the matrix $\mathcal{K}_{\sim \sim}^{\alpha}$ for the maximum of the free process.

\section{The Single Queue: Examples}

Many known models can be incorporated into the framework of the preceding section. To emphasize the versatility of our framework, we now give some examples. Importantly, the matrices that appear in these examples also play fundamental roles in a network setting; see Section 6.

The BMAP/GI/1 Queue The BMAP/GI/1 queue is a generalization of the classical $\mathrm{M} / \mathrm{GI} / 1$ queue. Here BMAP is shorthand for batch Markovian arrival process. Special cases include the MMPP/GI/1 queue, where MMPP stands for Markov modulated Poisson process, and the $\mathrm{PH} / \mathrm{GI} / 1$ queue, where $\mathrm{PH}$ stands for phase-type renewal process. For further special cases, we refer to Latouche and Ramaswami (1999, Sec. 3.5). The BMAP/GI/1 queue has been studied in detail by Lucantoni (1991), 
and it is our present aim to relate his results to ours. This is particularly relevant since our notation does not always agree with the standard notation in the matrix-analytic literature as used in Lucantoni (1991). We stress that none of the results presented here are new.

The virtual waiting time in a BMAP/GI/1 queue is defined as the buffer content in a fluid queue with special Markov-additive input; we describe this below. More precisely, as observed by Tzenova et al. (20005a), the BMAP/GI/1 queue can be viewed as a fluid-flow model with jumps (fluid-flow models are discussed below).

In a BMAP/GI/1 queue, the arrival process is governed by a Markovian background process $I$ that can take $N<\infty$ values. The sojourn time of $I$ in state $j$ has an exponential distribution with parameter $q_{j}$. At the end of a sojourn time in state $j$, with probability $p_{j k}^{(n)}, n \geq 0$ customers arrive (that all bring in a generic amount of work $U>0$ ) and a transition of $I$ to state $k$ occurs. These transition probabilities satisfy $\sum_{n=0}^{\infty} \sum_{k=1}^{N} p_{j k}^{(n)}=1$ for $j=1, \ldots, N$. We write $H$ for the distribution of $U$, and the stationary distribution of $I$ is denoted by $\pi_{I}$ as usual.

Let us now define the free process $X$ such that $(X, I)$ becomes a Markov-additive process, so that the setting of Section 4.2 can be used. Since the amount of work in the system decreases at unit rate, it readily follows that the Laplace exponent of $X$ is given by

$$
\boldsymbol{\psi}_{-X}(\beta)=\beta \boldsymbol{I}-\operatorname{diag}(q)\left(\boldsymbol{I}-\sum_{n=0}^{\infty} \boldsymbol{P}^{(n)}\left[\mathrm{E} e^{-\beta U}\right]^{n}\right),
$$

where $\boldsymbol{P}^{(n)}$ is the matrix with elements $p_{j k}^{(n)}$. We suppose that the system is stable, i.e., $\boldsymbol{\pi}_{I}^{\prime} \mathbf{E} X(1)<0$. It is an immediate consequence of Proposition 4.2 and the remarks thereafter that

$$
\mathrm{E} e^{-\omega W}=\omega \boldsymbol{u}_{-}^{\prime} \boldsymbol{\psi}_{-X}^{-1}(\omega) \mathbf{1},
$$

for $\omega \geq 0$ with $\psi_{-X}(\omega)$ nonsingular. This formula, in the present context due to Ramaswami, is Eq. 45 in Lucantoni (1991). In the matrix-analytic literature, it is customary to use the notation $\boldsymbol{y}_{0}$ for $\boldsymbol{u}_{-}$. Note that we have shown in Section 4.2 that $\boldsymbol{u}_{-}^{\prime} \mathcal{Q}_{\sim \sim}^{0}=\mathbf{0}_{-}^{\prime}$.

This motivates the investigation of the matrix $\mathcal{Q}_{\sim \sim}^{\alpha}$ for $\alpha \geq 0$. Upon setting

$$
\boldsymbol{G}^{\alpha}:=\int_{[0, \infty)} e^{\mathcal{Q}_{\sim \sim}^{\alpha} x} H(d x),
$$

we have by Proposition 3.2,

$$
\mathcal{Q}_{\sim \sim}^{\alpha}+\alpha \boldsymbol{I}=-\operatorname{diag}(q)\left(\boldsymbol{I}-\sum_{n=0}^{\infty} \boldsymbol{P}^{(n)}\left[\boldsymbol{G}^{\alpha}\right]^{n}\right) .
$$

Substitution of this expression in Eq. 25 leads to a fixed-point system for $\boldsymbol{G}^{\alpha}$ :

$$
\boldsymbol{G}^{\alpha}=\int_{[0, \infty)} e^{-\alpha x} e^{-\operatorname{diag}(q)\left(\boldsymbol{I}-\sum_{n=0}^{\infty} \boldsymbol{P}^{(n)}\left[\boldsymbol{G}^{\alpha}\right]^{n}\right) x} H(d x),
$$

which is the matrix version of Takacs' fixed-point equation if $\boldsymbol{P}^{(1)}$ is the only nonzero matrix in the sequence $\left\{\boldsymbol{P}^{(n)}: n \geq 0\right\}$. Based on this formula, Lucantoni (1991) gives 
an algorithm that serves as an efficient alternative for Neuts' approach to M/GI/1type queueing systems (Neuts 1989). Importantly, it is not necessary to compute $\mathcal{\mathcal { Q }}_{\sim \sim}^{0}$ in order to find $\boldsymbol{u}_{-}$: the definition of $\boldsymbol{G}^{0}$ in Eq. 25 shows that $\boldsymbol{u}_{-}$is necessarily proportional to the unique probability vector $\boldsymbol{g}$ satisfying $\boldsymbol{g}^{\prime} \boldsymbol{G}^{0}=\boldsymbol{g}^{\prime}$. The normalizing constant is found as in Section 4.2.

Fluid-flow Models A fluid-flow model is a fluid queue with a special type of Markovadditive input: the free process $X$ is neither allowed to have jumps nor Brownian states. They constitute undoubtedly the most well-studied fluid queues; we do not attempt to give a full bibliography, but refer to Asmussen (1995), Kulkarni (1997), Rogers (1994) for more details.

Recently, there has been some interest in deriving the Laplace transform of the busy period in fluid-flow models (Ahn and Ramaswami 2005; Bean et al. 2005b); see also (Asmussen 1994) for an earlier contribution. It is our present aim to show how our general theory reproduces some of the most important busy-period results. Thus, the results below are well-known. We remark that we allow states with zero drifts.

Even though fluid models are special Markov-additive processes, we shall work within the framework of Section 2 to derive formulas that are familiar from the fluidflow literature. To facilitate the use of our discrete-time results, we use an embedding that records the time and position at the beginning of a sojourn time of the underlying background process $I$. In self-evident notation, we partition the state space into +points, 0-points, and --points. The intensity matrix of $I$ is written as $\mathbf{Q}^{I}$; this also defines $\mathbf{Q}_{++}^{I}$, for instance.

Let $\Psi_{+-}^{\alpha}$ be the matrix with the transforms of the busy-period lengths. That is, if $c_{j}>0$ and $c_{k}<0$, then the element $(j, k)$ of this matrix is the Laplace transform of the length of the first positive excursion of $X$ on the event that it ends this excursion in state $k$. In other words, it corresponds to the amount of time that $X$ spends above zero on the event that it starts in state $j$ and it first hits zero in state $k$.

Let us use the notation $\operatorname{vec}\left(c_{+}\right)$and $\operatorname{vec}\left(c_{-}\right)$for the vector of strictly positive and strictly negative drifts respectively. We also set $\mu_{ \pm}^{\alpha}:=\operatorname{diag}\left(q_{ \pm} /\left(q_{ \pm}+\alpha\right)\right), \lambda_{ \pm}^{\alpha}:=$ $\operatorname{diag}\left(\left(q_{ \pm}+\alpha\right) / c_{ \pm}\right)$, and

$$
\begin{aligned}
& \mathbf{T}_{ \pm \pm}^{\alpha}:= \pm \operatorname{diag}\left(\frac{1}{c_{ \pm}}\right)\left[\mathbf{Q}_{ \pm \pm}^{I}-\alpha \boldsymbol{I}_{ \pm \pm}-\mathbf{Q}_{ \pm 0}^{I}\left(\mathbf{Q}_{00}^{I}-\alpha \boldsymbol{I}_{00}\right)^{-1} \mathbf{Q}_{0 \pm}^{I}\right] \\
& \mathbf{T}_{ \pm \mp}^{\alpha}:= \pm \operatorname{diag}\left(\frac{1}{c_{ \pm}}\right)\left[\mathbf{Q}_{ \pm \mp}^{I}-\mathbf{Q}_{ \pm 0}^{I}\left(\mathbf{Q}_{00}^{I}-\alpha \boldsymbol{I}_{00}\right)^{-1} \mathbf{Q}_{0 \mp}^{I}\right] .
\end{aligned}
$$

Note that, in the notation of Section 2, we are interested in $\boldsymbol{\Psi}_{+-}^{\alpha}=\boldsymbol{P}_{+-}^{\alpha} \operatorname{diag}\left(1 / \mu^{\alpha}\right)$. As in the proof of Corollary 2.1, we consider a sequence of +- and 0 -points as a single +-point, so that $\boldsymbol{F}_{+-}(\alpha, \beta)=\left(\beta \boldsymbol{I}_{++}-\mathbf{T}_{++}^{\alpha}\right)^{-1} \mathbf{T}_{+-}^{\alpha}$. Then Proposition 2.3 immediately yields that

$$
\boldsymbol{\Psi}_{+-}^{\alpha}=\int_{(0, \infty)} e^{\mathbf{T}_{++}^{\alpha} x} \mathbf{T}_{+-}^{\alpha} e^{\mathcal{Q}_{\sim \sim}^{\alpha} x} d x
$$

where $\mathcal{Q}_{\sim \sim}^{\alpha}=\mathbf{T}_{--}^{\alpha}+\mathbf{T}_{-+}^{\alpha} \Psi_{+-}^{\alpha}$. Since the eigenvalues of $\mathbf{T}_{++}^{\alpha}$ have a strictly negative real part and those of $\mathcal{Q}_{\sim \sim}^{\alpha}$ have a nonpositive real part, the integral in the above 
representation for $\Psi_{+-}^{\alpha}$ converges. This implies the identity (see Bean et al. 2005a, for references)

$$
\mathbf{T}_{++}^{\alpha} \Psi_{+-}^{\alpha}+\Psi_{+-}^{\alpha} \mathcal{Q}_{\sim \sim}^{\alpha}=-\mathbf{T}_{+-}^{\alpha} .
$$

After some rearranging and substitution of $\mathcal{Q}_{\sim \sim}^{\alpha}$, we obtain the matrix equation

$$
\mathbf{T}_{+-}^{\alpha}+\Psi_{+-}^{\alpha} \mathbf{T}_{-+}^{\alpha} \Psi_{+-}^{\alpha}+\mathbf{T}_{++}^{\alpha} \Psi_{+-}^{\alpha}+\Psi_{+-}^{\alpha} \mathbf{T}_{--}^{\alpha}=\mathbf{0}_{+-},
$$

which is Theorem 1 of Bean et al. (2005b) and, for $\alpha=0$, Theorem 2 of Rogers (1994). Note that no drift condition was imposed to derive this equation.

Importantly, the theory of Section 4.2 shows that the matrix $\mathcal{Q}_{\sim \sim}^{\alpha}$ is a key quantity for fluid-flow models. For instance, under a stability assumption, a left eigenvector of $\mathcal{Q}_{\sim \sim}^{0}$ (corresponding to the simple eigenvalue zero) appears in the representation of $W$ as a phase-type distribution. The matrix $\mathcal{Q}_{\sim \sim}^{\alpha}$ plays a prominent role in many system characteristics of fluid queues, see also Section 7.

$M / M / \infty$-driven Fluid Queues Although it was assumed that the state space of the background process be finite, we now give an example with a countably infinite state space (that, to the best of our knowledge, was not solved so far) that still fits into our framework. The model is a fluid-flow model, but we show that we can translate it in terms of the queue with Markov-modulated ON/OFF input of Section 4.1.

Consider the following queueing model. A buffer is emptied at a constant service rate $r$, and jobs arrive according to a Poisson process (with rate $\lambda$ ). They stay active for an exponentially distributed period of time (without loss of generality, we set its mean equal to 1); while active they feed work into the buffer at unit rate. Notice that the number of (active) jobs in the system follows an $\mathrm{M} / \mathrm{M} / \infty$-model, therefore it has a Poisson distribution with mean $\lambda$; denote $p_{k}:=e^{-\lambda} \lambda^{k} / k$ !. This leads to the stability condition $\lambda<r$.

The buffer level increases when the number of active jobs exceeds $r$, whereas the buffer is drained (or remains empty) when the number of jobs is below $r$. Let $X(t)$ denote the free process at time $t$ as before, and let $N(t)$ the number of active flows at time $t$. For ease we assume that $r \notin \mathbb{N} ; r_{-}:=\lfloor r\rfloor$ and $r_{+}:=\lceil r\rceil$. Define for $\ell \geq\lceil r\rceil$

$$
\sigma_{\ell}:=\inf \left\{t \geq 0: N(t)=r_{-} \mid N(0)=\ell\right\}, \quad U_{\ell}:=X\left(\sigma_{\ell}\right) .
$$

An explicit formula for $\xi_{\ell}(\alpha, \beta):=\mathbb{E}\left[e^{-\alpha \sigma_{\ell}-\beta U_{\ell}}\right]$ is provided by Preater (1997).

Due to exponentiality and reversibility properties, we have that the steady-state buffer content $W$ is distributed as $\sup _{t \geq 0} X(t)$. To study this supremum, it suffices to consider an embedding. One embedding could be the position of the free process at epochs jobs arrive and leave, but this has drawback that the dimension of the background process is (countably) infinite. Evidently, we could alternatively opt for the 'sparser' embedding that lumps together the states $r_{+}, r_{+}+1, \ldots$ into state $r_{+}$; the supremum of the embedded process coincides with the supremum of the full free process. Then the sojourn time in state $k=0, \ldots, r_{-}$is exponential with parameter $\lambda+k$, whereas the Laplace transform of the time spend in $r_{+}$, jointly with the net amount of work generated, is $\xi_{r_{+}}(\alpha, \beta)$. With $q_{j}:=\lambda+j$, it is easy to verify that corresponding discrete-time Markov chain on $\left\{0, \ldots, r_{+}\right\}$has the following transition 
probabilities: $p_{j, j+1}^{J}=\lambda / q_{j}$, if $j=0, \ldots, r_{-} ; p_{j, j-1}^{J}=j / q_{j}$, if $j=1, \ldots, r_{-} ; p_{r_{+}, r_{-}}^{J}=1$; $p_{j k}^{J}=0$, otherwise. Define $\mathbb{P}$ such that $(S, T, J)$ has the transition kernel

$$
\begin{aligned}
& p((s, t, j),(s+d v, t+d w, k)) \\
& \quad= \begin{cases}p_{j k}^{J} \mathbb{P}(U \in d v, \sigma \in d w) & \text { if } j=r_{+} \text {and } k=0, \ldots, r_{+} ; \\
p_{j k}^{J} \mathbb{P}\left(-D^{j} \in d v, \tau^{j} \in d w\right) \text { if } j=0, \ldots, r_{-} \text {and } k=0, \ldots, r_{+},\end{cases}
\end{aligned}
$$

with

$$
\mathbb{E} e^{-\alpha \sigma-\beta U}=\mathbb{E} e^{-\alpha \sigma_{r_{+}}-\beta U_{r_{+}}}=\xi_{r_{+}}(\alpha, \beta), \quad \mathbb{E} e^{-\alpha \tau^{j}-\beta D^{j}}=\frac{q_{j}}{q_{j}+\alpha+\beta(r-j)} .
$$

A procedure analogous to that for Markov-modulated ON/OFF input now yields for $k=0, \ldots, r_{-}$and $\omega, \beta \geq 0$,

$$
\mathbb{E}\left[e^{-\omega W-\beta B} ; I=k\right]=p_{k} \mathbb{E}_{k} e^{-\omega \bar{S}-\beta \bar{T}},
$$

and

$$
\mathbb{E}\left[e^{-\omega W-\beta B} ; I=r_{+}\right]=\left[\sum_{k=r_{+}}^{\infty} p_{k} \xi_{k}(\alpha, \beta)\right] \mathbb{E}_{r_{-}} e^{-\omega \bar{S}-\beta \bar{T}}
$$

\section{Tandem Networks with Markov-additive Input}

One of the simplest networks is a tandem network, in which $n$ fluid reservoirs are lined up in series. In this section, we extend the analysis of single stations to these tandem fluid networks. The results we obtain are new. Our analysis shows that we can immediately use the results on the joint distribution of the buffer content and the age of the busy period for the single queue, as found in Section 4. The reasoning below also shows that tandems with Markov-modulated ON/OFF input (Scheinhardt and Zwart 2002) can be analyzed analogously to tandems with Markov-additive input (Kella 2001); we here only present the analysis for Markov-additive input.

Even though our framework offers an appealing approach to such networks, we do not strive for the greatest possible generality. Instead, we only give the main ideas without proofs, since the results can be proven along the lines of Dębicki et al. (2007). Several extensions are discussed in the next section.

In our model queue $j$ is drained at rate $r_{j}$ as long as there is content in buffer $j$. After fluid is released from queue $j$, it immediately flows to queue $j+1$, unless $j=n$; then it leaves the system. We suppose that the input to the first queue is governed by the same Markov-additive process $(A, I)$ as in Section 4.2, i.e., its input process $A$ is spectrally positive. Furthermore, we suppose for simplicity that $I$ has no zerodrift states and that there is no external input to queues $2, \ldots, n$. To avoid 'invisible' stations, we impose the condition $r_{1}>\ldots>r_{n}$.

We define $W_{j}(t)$ as the content in buffer $j$ at time $t$, and let $\boldsymbol{W}(t)$ be the vector of buffer contents. The evolution of the process $\boldsymbol{W}$ is completely determined by $A$ and the initial buffer-content vector $\boldsymbol{W}(0)$. Formally, this can be made precise by using Skorokhod reflection mappings; see for instance Dębicki et al. (2007). It is our aim to study the steady-state vector of buffer contents in this network, which we denote 
by $\boldsymbol{W}:=\boldsymbol{W}(\infty)$. The inclusion of the ages of the busy periods raises no additional difficulties, but we focus here on the simplest possible situation.

We define for $j=1, \ldots, n, X_{j}(t):=A(t)-r_{j} t$ and $\boldsymbol{X}(t)=\left(X_{1}(t), \ldots, X_{n}(t)\right)^{\prime}$. Note that $(\boldsymbol{X}, I)$ is a multidimensional Markov-additive process on $\mathbb{R}^{n} \times\{1, \ldots, N\}$ under P. We also set

$$
\bar{X}_{j}:=\sup _{t \geq 0} X_{j}(t), \quad \bar{F}_{j}^{X}:=\inf \left\{t \geq 0: X_{j}(t)=\bar{X}_{j}(\infty) \text { or } X_{j}(t-)=\bar{X}_{j}(\infty)\right\},
$$

and $\bar{I}_{j}:=I\left(\bar{F}_{j}^{X}\right)$. Throughout, we suppose that $\pi_{I}^{\prime} \mathbf{E} X_{n}(1)<0$, so that each component of $\boldsymbol{X}$ drifts to $-\infty$.

Our analysis consists of three steps. First, the queueing problem is formulated in terms of free processes. The splitting technique of Section 3.2 can be used, in a different form, to characterize the extremes of these free processes. This is reminiscent of the analysis of Lévy-driven fluid networks in Dębicki et al. (2007). The final step converts the results back to the queueing setting.

We start by giving the analog of Proposition 4.2, thereby establishing the connection between fluid networks and extremes of $X$. It can be proven along the lines of Proposition 5.2 in Dȩbicki et al. (2007). Note that the distribution of $\boldsymbol{W}=\boldsymbol{W}(\infty)$ is independent of $\boldsymbol{W}(0)$ and $I(0)$.

Proposition 6.1 The vector $\boldsymbol{W}$ is finite, and for any $\omega \in \mathbb{R}_{+}^{n}$, we have

$$
\mathrm{E}\left[e^{-\langle\omega, \boldsymbol{W}\rangle} ; I=k\right]=\boldsymbol{\pi}_{I}(k) \widehat{\mathrm{E}}_{k}\left[e^{-\sum_{i=1}^{n-1}\left(\omega_{i}-\omega_{i+1}\right) \bar{X}_{i}-\omega_{n} \bar{X}_{n}} ; \bar{I}_{n}\right] \mathbf{1} .
$$

We use splitting to calculate the transform in this expression. In Dębicki et al. (2007), splitting is distinguished from splitting from the left, but this is irrelevant for the arguments and the results. Modulo this remark, the following lemma can be proven along the lines of Lemma 2.1 of Dȩbicki et al. (2007).

Lemma 6.1 For any $j, \quad\left\{(\boldsymbol{X}(t), I(t)): 0 \leq t \leq \bar{F}_{j}^{X}\right\} \quad$ and $\quad\left\{\left(\boldsymbol{X}\left(\bar{F}_{j}^{X}+t\right)-\boldsymbol{X}\left(\bar{F}_{j}^{X}\right)\right.\right.$, $\left.\left.I\left(\bar{F}_{j}^{X}+t\right)\right): t \geq 0\right\}$ are $\widehat{\mathrm{P}}$-conditionally independent given $I\left(\bar{F}_{j}^{X}\right)$.

With this proposition at our disposal, the joint distribution of $\overline{\boldsymbol{F}}^{X}:=\left(\bar{F}_{1}^{X}, \ldots, \bar{F}_{n}^{X}\right)$ and $\overline{\boldsymbol{X}}:=\left(\bar{X}_{1}, \ldots, \bar{X}_{n}\right)$ can be derived in only a few lines. The key element in this analysis is the observation $\bar{F}_{1}^{X} \leq \ldots \leq \bar{F}_{n}^{X}$. In the following theorem, we give the resulting Laplace transform; in the terminology of Dębicki et al. (2007), this transform has a quasi-product form. The proof requires only minor modifications in comparison with the proof of Theorem 3.1 of Dȩbicki et al. (2007), and is therefore omitted. We emphasize that the product is taken from 1 to $n-1$; the order is important, since the matrices do not commute. 
Corollary 6.1 We have for $\boldsymbol{\beta} \in \mathbb{R}_{+}^{n}$,

$$
\begin{aligned}
\widehat{\mathbf{E}}\left[e^{-\langle\boldsymbol{\beta}, \overline{\boldsymbol{X}}\rangle} ; \bar{I}_{n} \in \sim\right]= & \widehat{\mathbf{E}}\left[e^{-\left[\sum_{k=2}^{n}\left(r_{1}-r_{k}\right) \beta_{k}\right] \bar{F}_{1}^{X}-\left[\sum_{k=1}^{n} \beta_{k}\right] \bar{X}_{1}} ; \bar{I}_{1} \in \sim\right] \\
& \times \prod_{j=1}^{n-1}\left\{\left(\widehat{\mathbf{E}}_{\sim}\left[e^{-\left[\sum_{k=j+1}^{n}\left(r_{j}-r_{k}\right) \beta_{k}\right] \bar{F}_{j}^{X}-\left[\sum_{k=j+1}^{n} \beta_{k}\right] \bar{X}_{j}} ; \bar{I}_{j} \in \sim\right]\right)^{-1}\right. \\
& \left.\times \widehat{\mathbf{E}}_{\sim}\left[e^{-\left[\sum_{k=j+2}^{n}\left(r_{j+1}-r_{k}\right) \beta_{k}\right] \bar{F}_{j+1}^{X}-\left[\sum_{k=j+1}^{n} \beta_{k}\right] \bar{X}_{j+1}} ; \bar{I}_{j+1} \in \sim\right]\right\},
\end{aligned}
$$

whenever the appropriate matrices are nonsingular.

Corollary 6.1 expresses the transform of the $\widehat{\mathrm{P}}$-distribution of $\left(\overline{\boldsymbol{X}}, \bar{I}_{n}\right)$ in terms of the marginals $\left(\bar{X}_{j}, \bar{I}_{j}\right)$ for $j=1, \ldots, n$. Importantly, the transforms of these marginals can be found with Corollary 4.1. As a final step, we therefore cast the results back into the queueing setting. For notational convenience, we define

$$
\eta_{j}(\omega):=\sum_{\ell=j+1}^{n}\left(r_{\ell-1}-r_{\ell}\right) \omega_{\ell},
$$

so that we obtain the main result of this section, which is a generalization of Eq. 23. The simplicity of the expression for the Laplace transform is remarkable, especially in view of the transform-free solution of Kroese and Scheinhardt (2001) for the twostation fluid-flow tandem with a two-dimensional background state space. The matrix $\mathcal{Q}_{\sim \sim}^{(j)}(\alpha)$ appearing in the following theorem is defined as the $\mathcal{Q}_{\sim \sim}^{\alpha}$-matrix arising from the process $X_{j}$.

Theorem 6.1 For $\omega \in \mathbb{R}_{+}^{n}$, we have

$$
\begin{aligned}
\mathrm{E} & {\left[e^{-\langle\boldsymbol{\omega}, \boldsymbol{W}\rangle} ; I\right] } \\
= & \left(\boldsymbol{0}_{s}^{\prime} \omega_{n}\left[\boldsymbol{u}_{-}^{n}\right]^{\prime} \prod_{j=1}^{n-1}\left\{\left[\omega_{j+1} \boldsymbol{I}_{--}+\mathcal{Q}_{\sim \sim}^{(j)}\left(\eta_{j}(\boldsymbol{\omega})\right)\right]^{-1}\left[\omega_{j} \boldsymbol{I}_{--}+\mathcal{Q}_{\sim \sim}^{(j)}\left(\eta_{j}(\boldsymbol{\omega})\right)\right]\right\}\right) \\
& \times\left(\boldsymbol{\psi}-X_{1}\left(\omega_{1}\right)-\eta_{1}(\boldsymbol{\omega}) \boldsymbol{I}\right)^{-1},
\end{aligned}
$$

whenever the appropriate matrices are nonsingular.

Importantly, this theorem shows that the joint buffer-content distribution for a fluid network can immediately be established from known results about the single (fluid) queue discussed in Section 5. For instance, Lucantoni's algorithm for the BMAP/GI/1 immediately yields $\mathcal{Q}_{\sim \sim}^{(j)}(\cdot)$, and similarly for algorithms that efficiently solve the matrix-quadratic equation in fluid-flow models.

Specializing Theorem 6.1 to the marginal distribution of $W_{n}$ for $n>1$, we obtain the interesting formula

$$
\mathrm{E}\left[e^{-\omega W_{n}} ; I \in \sim\right]=\frac{\left[\boldsymbol{u}_{-}^{n}\right]^{\prime}}{r_{n}-r_{n-1}}\left[\omega \boldsymbol{I}_{--}+\mathcal{Q}_{\sim \sim}^{(n-1)}\left(\left(r_{n-1}-r_{n}\right) \omega\right)\right]^{-1} \mathcal{Q}_{\sim \sim}^{(n-1)}\left(\left(r_{n-1}-r_{n}\right) \omega\right),
$$


which should be compared with Theorem 3.2 of Dȩbicki et al. (2007) or Corollary 6.2(i) of Dȩbicki et al. (2007).

\section{Extensions}

In the course of writing this paper, we have bypassed several interesting questions. It is the aim of this section to sketch how some additional features can be incorporated into our framework. These features are mainly inspired by models that have been recently studied in the literature.

Markov-additive Processes under Exponential Killing The approach taken in this paper can also be used to characterize the distributions of $\left(\bar{X}(t), \bar{F}^{X}(t), \bar{I}(t)\right)$ and $\left(\bar{X}(t), \bar{F}^{X}(t), I(t)\right)$ for any $t \geq 0$. By taking Laplace transforms with respect to time, this amounts to investigating $\left(\bar{X}\left(e_{\lambda}\right), \bar{F}^{X}\left(e_{\lambda}\right), \bar{I}\left(e_{\lambda}\right)\right)$ and $\left(\bar{X}\left(e_{\lambda}\right), \bar{F}^{X}\left(e_{\lambda}\right), I\left(e_{\lambda}\right)\right)$ for some $\lambda>0$. The resulting identities can be viewed as the analog of Eq. 11 if $X$ is spectrally positive.

The vector $\left(\bar{X}\left(e_{\lambda}\right), I\left(e_{\lambda}\right)\right)$ plays a role in a number of problems in applied probability. First, it completely specifies the solution to the one-sided exit problem (Kyprianou and Palmowski 2008). We remark that, if there are no subordinator states, the nonnegative matrix $-\left(\mathcal{K}_{\sim \sim}^{\lambda}\right)^{-1}$ plays a prominent role in this solution; it can be interpreted as a local-time matrix. Moreover, the distribution of $\left(\bar{X}\left(e_{\lambda}\right), I\left(e_{\lambda}\right)\right)$ also immediately specifies the transient behavior of a queue with Markov-additive input, see Ahn and Ramaswami (2005) for a special case.

Ramifications of the Tandem Network in Section 6; Priority Systems In Section 6, there are no external inputs to the stations $2, \ldots, n$ of a tandem fluid network. As long as these external inputs are increasing subordinators, i.e., if they do not depend on the state of the background process $I$, our reasoning immediately carries over to this more general setting.

Kella (2001) does allow for a dependence of this external input (or the drain rates) on the background state, and we now outline how our framework should be modified to be able to derive expressions under this assumption. In terms of the onedimensional Markov-additive process $X$ of Section 3, it is not sufficient to study $\bar{F}^{X}$ (jointly with $(\bar{X}, \bar{I})$ ), but knowledge is required about the amount of time spent in each of the states till time $\bar{F}^{X}$.

The last-passage (or Wiener-Hopf) approach that we have used in this paper can still be applied, but the matrices $\mathcal{K}_{\sim \sim}^{\alpha}$ now depend on a vector $\operatorname{vec}(\alpha)$ instead of a single value. An expression such as $\psi_{-X}(\beta)-\alpha \boldsymbol{I}$ in Theorem 3.1 then changes to $\psi_{-X}(\beta)-\operatorname{diag}(\alpha)$. However, the reasoning essentially requires no further new ideas. As for tandem networks, the only remaining assumption is that the components of $\overline{\boldsymbol{F}}^{X}$ are ordered (note that a similar assumption is needed in Kella 2001).

Recently, there has been an interest in fluid-driven priority systems (Takada and Miyazawa 2002; Tzenova et al. 20005b). These systems are closely related tandem queues with external inputs and equal drain rates. Although equal drain rates are not covered in Section 6, the techniques still apply. Indeed, if the external inputs are nondecreasing processes (with the first station as the only possible exception, see for 
instance Dȩbicki et al. 2007), the components of $\overline{\boldsymbol{F}}^{X}$ are ordered. In particular, our theory can be used to analyze priority fluid systems with Markov-additive input.

Phase-type Jumps in the Opposite Direction All Markov-additive processes in this paper have one-sided jumps. Given the tractability of Lévy processes with general jumps in one direction and phase-type jumps in the other direction (Dieker 2006), it seems plausible that results can be obtained within the Markov-additive setting under the same assumptions. Indeed, an embedded process can be introduced and the theory of Section 2 can be applied.

Acknowledgements We are grateful to Offer Kella and Peter Taylor for useful discussions. We also thank the anonymous referees for their careful reading.

ABD was partially supported by the Netherlands Organization for Scientific Research (NWO), grant number 631.000.002, and the Science Foundation Ireland, grant number SFI04/RP1/I512.

Open Access This article is distributed under the terms of the Creative Commons Attribution Noncommercial License which permits any noncommercial use, distribution, and reproduction in any medium, provided the original author(s) and source are credited.

\section{Appendix: The Spectral Method for the Matrices $K_{--}^{\alpha}$ and $Q_{--}^{\alpha}$}

Corollaries 2.1 and 2.2 give two non-linear matrix equations that must be satisfied by $\boldsymbol{K}_{--}^{\alpha}$ and $\boldsymbol{Q}_{---}^{\alpha}$. This appendix describes and analyzes an alternative method to find these two matrices. To our knowledge, the resulting approach is novel.

Exactly the same approach can be taken in the context of the Markov-additive matrices $\mathcal{K}_{\sim \sim}^{\alpha}$ and $\mathcal{Q}_{\sim \sim}^{\alpha}$, but we here focus on the discrete-time framework of Section 2. Throughout, we fix some $\alpha \geq 0$ and we suppose that $S$ drifts to $+\infty$ or $-\infty$.

As observed in the body of this paper, subsequent +-points may be 'lumped' in order to calculate the matrices $\boldsymbol{K}_{--}^{\alpha}$ and $\boldsymbol{Q}_{--}^{\alpha}$. Therefore, if we replace $F_{+-}(\alpha, \beta)$ by $\boldsymbol{F}_{+\circlearrowleft_{-}}(\alpha, \beta)$, we may assume without loss of generality that $\boldsymbol{P}_{++}^{J}=\boldsymbol{0}_{++}$. The reasoning that led to Eq. 6 shows that $\boldsymbol{D}_{--}(0, \beta)$ then factorizes into two matrices:

$$
\boldsymbol{D}_{--}(\alpha, \beta)=\left(\beta \boldsymbol{I}_{--}+\boldsymbol{K}_{--}^{\alpha}\right)\left(\boldsymbol{I}_{--}-\boldsymbol{E}_{-}\left[e^{-\alpha T_{\tau_{+}}-\beta S_{\tau_{+}}} ; J_{\tau_{+}} \in-\right]\right) .
$$

This equation can be regarded as a factorization identity, and is the starting point of the spectral method. When inspecting the two matrices enclosed by round brackets on the right-hand side, we note that the first matrix has singularities in the right complex halfplane and the second matrix in the left complex halfplane. For similar factorizations in a discrete-state framework, we refer to Zhao et al. (2003).

A similar factorization can be given for $\boldsymbol{Q}_{--}^{\alpha}$ : the first-passage matrix $\boldsymbol{Q}_{--}^{\alpha}$ of the original process can be expressed in terms of the last-passage matrix $\widehat{\boldsymbol{K}}_{--}^{\alpha}$ of the time-reversed process through

$$
\widehat{\boldsymbol{K}}_{--}^{\alpha}=\operatorname{diag}\left(\lambda^{\alpha}\right)^{\#} \boldsymbol{Q}_{--}^{\alpha} \operatorname{diag}\left(\lambda^{\alpha}\right)^{-1},
$$

cf. Eq. 3. An analysis along the lines of Section 2 yields the factorization identity

$$
\operatorname{diag}\left(\lambda^{\alpha}\right){ }^{\#} \boldsymbol{D}_{--}(\alpha, \beta) \operatorname{diag}\left(\lambda^{\alpha}\right)^{-1}=\left(\beta \boldsymbol{I}_{--}+\widehat{\boldsymbol{K}}_{--}^{\alpha}\right) \boldsymbol{N}(\alpha, \beta),
$$


where $\mathbf{N}(\alpha, \beta)$ is an $N_{-} \times N_{-}$-matrix with singularities in the left complex halfplane. This yields a second factorization identity:

$$
\boldsymbol{D}_{--}(\alpha, \beta)=\operatorname{diag}\left(\lambda^{\alpha}\right){ }^{\#} \boldsymbol{N}(\alpha, \beta) \operatorname{diag}\left(\lambda^{\alpha}\right)^{-1}\left(\beta \boldsymbol{I}_{--}+\boldsymbol{Q}_{--}^{\alpha}\right) .
$$

The spectral method uses Eq. 26 or Eq. 27 to construct $\boldsymbol{K}_{--}^{\alpha}$ and $\boldsymbol{Q}_{--}^{\alpha}$ from their eigenvalues and eigenvectors. We explain the key ideas by discussing the following proposition, which is a special case of Theorem 8.1 below. It immediately follows from Eqs. 26 and 27, see also Section 5 of Asmussen (1995) for related results. Recall the notation $\mathcal{H}_{+}$from Eq. 4 .

Proposition 8.1 For any $v \in \mathcal{H}_{+}$, the following are equivalent:

(i) $-v$ is an eigenvalue of $\boldsymbol{Q}_{--}^{\alpha}$,

(ii) $-v$ is an eigenvalue of $\boldsymbol{K}_{--}^{\alpha}$, and

(iii) zero is an eigenvalue of $\boldsymbol{D}_{--}(\alpha, v)$.

Moreover, the geometric multiplicities of these eigenvalues coincide.

Proposition 8.1 indicates why the recursions in Corollaries 2.1 and 2.2 are necessarily matrix versions of the equation $\boldsymbol{D}_{--}(\alpha, \beta)=\mathbf{0}_{--}$. Indeed, suppose that $(-v, \ell)$ is a left eigenpair for $\boldsymbol{K}_{--}^{\alpha}$, so that $\boldsymbol{\ell}^{\prime} \boldsymbol{K}_{--}^{\alpha}=-v \boldsymbol{\ell}^{\prime}$. Since then $\boldsymbol{\ell}^{\prime} e^{\boldsymbol{K}_{--}^{\alpha}{ }^{x}}=e^{-v x} \boldsymbol{\ell}^{\prime}$, it follows from the recursion for $\boldsymbol{K}_{--}^{\alpha}$ in Corollary 2.1 that $\boldsymbol{\ell}^{\prime} \boldsymbol{D}_{--}(\alpha, v)=\mathbf{0}_{-}^{\prime}$. The same reasoning goes through for the recursion in Corollary 2.2, but one then has to work with the right eigenpair.

If $\boldsymbol{K}_{--}^{\alpha}$ or $\boldsymbol{Q}_{--}^{\alpha}$ is diagonalizable, Proposition 8.1 shows that its eigenvalues and eigenvectors (and hence the matrix itself) can be determined by studying singularities of $\beta \mapsto \boldsymbol{D}_{--}(\alpha, \beta)$, i.e., the values of $\beta$ for which this matrix is singular. Several relatively explicit results can then be derived, see Kella (2001). However, if $\boldsymbol{K}_{--}^{\alpha}$ is not diagonalizable, Proposition 8.1 shows that it is impossible to find enough pairs $\left(-v_{j}, \boldsymbol{\ell}_{j}\right)$ with the above properties. To resolve this, one might guess that the generalized left eigenvectors of $\boldsymbol{D}_{--}\left(\alpha, v_{j}\right)$ can be used to construct $\boldsymbol{K}_{--}^{\alpha}$. It is the contribution of this appendix to show that this approach does not work, and to show how this can be resolved. In particular, we provide answers to the questions raised in Section 4 of Asmussen and Kella (2000) in the continuous-time Markov-additive context.

Proposition 8.1 has implications for the locations of the singularities of $\boldsymbol{D}_{--}(\alpha, \beta)$ in $\mathcal{H}_{+}$. First, since $\boldsymbol{K}_{--}^{\alpha}$ and $\boldsymbol{Q}_{--}^{\alpha}$ are real matrices, these singularities must come in conjugate pairs. Moreover, as a result of Proposition 2.4, if zero is a singularity it is simple and the real parts of the other singularities are strictly positive. In fact, all nonzero singularities must be in the open disc with radius and center $\max _{j} \lambda_{j}^{\alpha}$. For $\alpha=0$ and $\lim _{n} S_{n}=-\infty$, this claim has recently been proven with different methods by Tzenova et al. (20005a). In Tzenova et al. (20005a), it is also shown that $\beta \mapsto$ det $\boldsymbol{D}_{--}(0, \beta)$ has exactly $N_{-}$zeroes in $\mathcal{H}_{+}$(counting multiplicities).

If $S$ drifts to $-\infty$, Proposition 8.1 can sometimes be used to find the vector $\boldsymbol{P}_{-}(\bar{S}=0)$ studied in Section 2.4. Indeed, in view of Lemma 2.3, $\boldsymbol{P}_{-}(\bar{S}=0)$ can be found if one has $N_{-}-1$ linear independent vectors $\ell_{1}, \ldots, \ell_{N_{-}-1}$ orthogonal to $\boldsymbol{P}_{-}(\bar{S}=0)$. To determine the vectors $\boldsymbol{\ell}_{j}$, one determines a root $v_{j} \in \mathcal{H}_{+}$of the equation det $\boldsymbol{D}_{--}(0, \beta)=0$, and identifies the $\boldsymbol{\ell}_{j}$ with a left eigenvector of $\boldsymbol{D}_{--}\left(0, v_{j}\right)$ 
corresponding to the eigenvalue zero. By Corollary 2.4 we then have $\boldsymbol{\ell}_{j}^{\prime} \boldsymbol{P}_{-}(\bar{S}=0)=$ 0 . Proposition 8.1 shows that enough independent vectors can be found only if $\boldsymbol{K}_{--}^{0}$ (or $\boldsymbol{Q}_{--}^{0}$ ) is diagonalizable.

As an aside, we mention that Gail et al. (1996) present a method (in the context of a discrete-state model) for determining the vector $\boldsymbol{P}_{-}(\bar{S}=0)$ if $S$ drifts to $-\infty$, and that they also call this a 'spectral method'. Cast into the present setting, they show that adj $\boldsymbol{D}_{--}(0, \beta) \boldsymbol{P}_{-}(\bar{S}=0)$ must vanish to the order at least $r$ at $\beta=v$ if $v \neq 0$ is a singularity of $\boldsymbol{D}_{--}(0, v)$ with algebraic multiplicity $r$. Here adj $\boldsymbol{D}_{--}(0, \beta)$ denotes the adjoint matrix of $\boldsymbol{D}_{--}(0, \beta)$, i.e., the transpose of the matrix formed by taking the cofactor of each element of $\boldsymbol{D}_{--}(0, \beta)$.

It is the aim of the remainder of the appendix to find a suitable form of the spectral method with which $\boldsymbol{K}_{--}^{\alpha}$ and $\boldsymbol{Q}_{--}^{\alpha}$ can always be constructed, not only in the diagonalizable case. If $S$ drifts to $-\infty$ and $\alpha=0$, the procedure also gives exactly $N_{-}-1$ vectors orthogonal to $\boldsymbol{P}_{-}(\bar{S}=0)$.

It is most insightful to present the procedure in an algorithmic form:

- Locate the singularities of $\boldsymbol{D}_{--}(\alpha, \beta)$ in $\mathcal{H}_{+}$(if $\lim _{n} S_{n}=-\infty$ and $\alpha=0$, then $\beta=0$ is such a singularity).

- For every nonzero singularity $v$, find as many independent vectors $\ell$ with $\boldsymbol{\ell}^{\prime} \boldsymbol{D}_{--}(\alpha, v)=\mathbf{0}_{-}^{\prime}$ as possible (if $\lim _{n} S_{n}=-\infty$ and $\alpha=0$, then $\boldsymbol{\pi}_{-}$is such a vector for $v=0$, see Eq. 8).

- This results in $s$ pairs $\left(-v_{j}, \ell_{j}\right)$, for some $s \leq N_{-}, j=1, \ldots, s$ (the $v_{j}$ need not be distinct). If $s=N_{-}$, then stop; $\boldsymbol{K}_{--}^{\alpha}$ is diagonalizable.

- Suppose that $\boldsymbol{K}_{--}^{\alpha}$ is not diagonalizable. If $\lim _{n} S_{n}=+\infty$ or $\alpha>0$, execute the following subroutine for each $j=1, \ldots, s$. If $\lim _{n} S_{n}=-\infty$ and $\alpha=0$, set $d_{s}=1$ and $\boldsymbol{\ell}_{s}^{(1)}=\pi_{-}$, and execute the following subroutine for each $j=1, \ldots, s-1$ :

- Set $p:=1$ and write $\boldsymbol{\ell}_{j}^{(1)}:=\boldsymbol{\ell}_{j}$.

- If possible, find a vector $\ell$, independent of $\ell_{j}^{(1)}, \ldots, \ell_{j}^{(p)}$, such that

$$
\boldsymbol{\ell}^{\prime} \boldsymbol{D}_{--}\left(\alpha, v_{j}\right)=\boldsymbol{\ell}_{j}^{(p)^{\prime}}-\sum_{q=1}^{p} \int_{[0, \infty)} \frac{x^{q}}{q !} e^{-v_{j} x} \boldsymbol{\ell}_{j}^{(p-q+1)^{\prime}} \operatorname{diag}\left(\mu^{\alpha} \lambda^{\alpha}\right) \boldsymbol{P}_{-+}^{J} \boldsymbol{F}_{+\circlearrowleft-}^{\alpha}(d x) .
$$

- If the previous step was successful, set $\ell_{j}^{(p+1)}:=\ell, p=p+1$, and repeat the previous step. If it was unsuccessful, set $d_{j}:=p$ and stop the subroutine.

The following theorem shows that this algorithm yields $\boldsymbol{K}_{--}^{\alpha}$ for $\alpha \geq 0$, in addition to $\boldsymbol{P}_{-}(\bar{S}=0)$ if $S$ drifts to $-\infty$. The matrix $\boldsymbol{Q}_{--}^{\alpha}$ can be found in a similar fashion, using Eq. 27 as a starting point. For notational convenience, we only write down the nonzero elements of the matrices. Note that the $\boldsymbol{J}_{j}$-matrices are Jordan blocks.

Theorem 8.1 For $\alpha \geq 0$, the matrix $\boldsymbol{K}_{--}^{\alpha}$ is constructed as follows:

$$
\boldsymbol{K}_{--}^{\alpha}=\left(\begin{array}{c}
\boldsymbol{L}_{1} \\
\vdots \\
\boldsymbol{L}_{s}
\end{array}\right)^{-1}\left(\begin{array}{ccc}
\boldsymbol{J}_{1} & & \\
& \ddots & \\
& & \boldsymbol{J}_{s}
\end{array}\right)\left(\begin{array}{c}
\boldsymbol{L}_{1} \\
\vdots \\
\boldsymbol{L}_{s}
\end{array}\right)
$$


where the $\left(d_{j} \times d_{j}\right)$-matrices $\boldsymbol{J}_{j}$ and $\left(d_{j} \times N_{-}\right)$-matrices $\boldsymbol{L}_{j}$ are defined as

$$
\boldsymbol{J}_{j}:=\left(\begin{array}{cccc}
-v_{j} & & & \\
1 & -v_{j} & & \\
& \ddots & \ddots & \\
& & 1 & -v_{j}
\end{array}\right), \quad \boldsymbol{L}_{j}=\left(\begin{array}{c}
\boldsymbol{\ell}_{j}^{(1)^{\prime}} \\
\vdots \\
\boldsymbol{\ell}_{j}^{\left(d_{j}\right)^{\prime}}
\end{array}\right) .
$$

Moreover, if $\lim _{n} S_{n}=-\infty$ and $\alpha=0$, then the rows of $\boldsymbol{L}_{1}, \ldots, \boldsymbol{L}_{s-1}$ constitute exactly $N_{-}-1$ independent vectors orthogonal to $\boldsymbol{P}_{-}(\bar{S}=0)$.

Proof If suffices to prove the first claim, since the second claim immediately follows from Eq. 7. For convenience, we denote the second matrix between round brackets in Eq. 26 by $\boldsymbol{M}(\alpha, \beta)$.

To prove the theorem, write $\boldsymbol{K}_{--}^{\alpha}$ in the Jordan form $\boldsymbol{L}_{--}^{-1} \boldsymbol{J}_{--} \boldsymbol{L}_{--}$, cf. Eq. 28. If $\lim _{n} S_{n}=-\infty$ and $\alpha=0$, we know that zero is a simple eigenvalue and that its corresponding left eigenvector is $\pi_{-}$, cf. Proposition 2.4. Factorization identity Eq. 26 shows that

$$
\operatorname{adj}\left(\beta \boldsymbol{I}_{--}+\boldsymbol{J}_{--}\right) \boldsymbol{L}_{--} \boldsymbol{D}_{--}(\alpha, \beta)=\operatorname{det}\left(\beta \boldsymbol{I}_{--}+\boldsymbol{J}_{--}\right) \boldsymbol{L}_{--} \boldsymbol{M}(\alpha, \beta) .
$$

Now observe that $\beta \boldsymbol{I}_{--}+\boldsymbol{J}_{--}$is a block-diagonal matrix, and that for (square) block matrices $\boldsymbol{A}$ and $\boldsymbol{B}$ of arbitrary size,

$$
\operatorname{adj}\left(\begin{array}{ll}
\boldsymbol{A} & \mathbf{0} \\
\mathbf{0} & \boldsymbol{B}
\end{array}\right)=\left(\begin{array}{cc}
\operatorname{det} \boldsymbol{B} \operatorname{adj} \boldsymbol{A} & \mathbf{0} \\
\mathbf{0} & \operatorname{det} \boldsymbol{A} \operatorname{adj} \boldsymbol{B}
\end{array}\right) .
$$

This shows that Eq. 29 is equivalent to the $s$ systems

$$
\operatorname{adj}\left(\beta \boldsymbol{I}_{d_{j} d_{j}}+\boldsymbol{J}_{j}\right) \boldsymbol{L}_{j} \boldsymbol{D}_{--}(\alpha, \beta)=\left(\beta-v_{j}\right)^{d_{j}} \boldsymbol{L}_{j} \boldsymbol{M}(\alpha, \beta) .
$$

If $\alpha=0$, the equation for $j=s$ plays no role and is redundant. In the rest of the proof, we consider this system for fixed $j$ and suppress the subscripts $j$.

It remains to show that our algorithm constructs the matrix $\boldsymbol{L}\left(\equiv \boldsymbol{L}_{j}\right)$. First observe that Eq. 30 is equivalent to the $d$ equations

$$
\sum_{p=1}^{n} \frac{(-1)^{p-1}}{(\beta-v)^{n-p}} \boldsymbol{\ell}^{(p)^{\prime}} \boldsymbol{D}_{--}(\alpha, \beta)=(\beta-v) \boldsymbol{\ell}^{(n)^{\prime}} \boldsymbol{M}(\alpha, \beta),
$$

for $n=1, \ldots, d$ and $\beta \geq 0$. For notational convenience, we set

$$
\boldsymbol{D}_{--}^{(q)}(\alpha, v):=\int_{[0, \infty)} \frac{x^{q}}{q !} e^{-v x} \operatorname{diag}\left(\mu^{\alpha} \lambda^{\alpha}\right) \boldsymbol{P}_{-+}^{J} \boldsymbol{F}_{+{ }^{-}}^{\alpha}(d x) .
$$

We now prove:

Claim A Let $1 \leq k \leq d$. If Eq. 31 holds for $n=1, \ldots, k$ and $\beta \geq 0$, then $\boldsymbol{\ell}^{(1)^{\prime}} \boldsymbol{D}_{--}(\alpha, \beta)=\mathbf{0}_{-}^{\prime}$ and

$$
\boldsymbol{\ell}^{(n)^{\prime}} \boldsymbol{D}_{--}(\alpha, v)=\boldsymbol{\ell}^{(n-1)^{\prime}}-\sum_{q=1}^{n-1} \boldsymbol{\ell}^{(q)^{\prime}} \boldsymbol{D}_{--}^{(n-q)}(\alpha, v)
$$

for $n=2, \ldots, k$. 
To see that Claim A is true for $k=2$, set $n=1$ in Eq. 31 and let $\beta \rightarrow v$ to obtain $\boldsymbol{\ell}^{(1)^{\prime}} \boldsymbol{D}_{--}(\alpha, v)=\mathbf{0}_{-}$. Using Eq. 31 for $n=2$, we see that

$$
\boldsymbol{\ell}^{(2)^{\prime}} \boldsymbol{D}_{--}(\alpha, \beta)-\frac{1}{\beta-v} \boldsymbol{\ell}^{(1)^{\prime}}\left[\boldsymbol{D}_{--}(\alpha, \beta)-\boldsymbol{D}_{--}(\alpha, v)\right]=(\beta-v) \boldsymbol{\ell}^{(2)^{\prime}} \boldsymbol{M}(\alpha, \beta) .
$$

Upon letting $\beta \rightarrow v$, we see (with dominated convergence and $\Re(v)>0$ ) that $\boldsymbol{\ell}^{(2)^{\prime}} \boldsymbol{D}_{--}(\alpha, v)=\boldsymbol{\ell}^{(1)^{\prime}}-\boldsymbol{\ell}^{(1)^{\prime}} \boldsymbol{D}_{--}^{(1)}(\alpha, v)$.

Suppose that Claim A holds for some $k$; by induction it suffices to show that it also holds for $k+1$. For this, first multiply the $k-1$ equations in Eq. 32 by $(-1)^{n-1}(\beta-$ $v)^{n-k-1}$, and substitute them in Eq. 31 for $n=k+1$ such that terms $\boldsymbol{D}_{--}(\alpha, \beta)-$ $\boldsymbol{D}_{--}(\alpha, v)$ appear everywhere; also use $\boldsymbol{\ell}^{(1)^{\prime}} \boldsymbol{D}_{--}(\alpha, v)=\mathbf{0}_{-}^{\prime}$. After some algebra, one then obtains

$$
\begin{aligned}
(\beta-v) \boldsymbol{\ell}^{(k+1)^{\prime}} \boldsymbol{M}(\alpha, \beta)= & (-1)^{k} \boldsymbol{\ell}^{(k+1)^{\prime}} \boldsymbol{D}_{--}(\alpha, \beta) \\
& +\frac{(-1)^{k-1}}{\beta-v} \boldsymbol{\ell}^{(k)^{\prime}}\left[\boldsymbol{D}_{--}(\alpha, \beta)-\boldsymbol{D}_{--}(\alpha, v)\right] \\
& +\sum_{n=1}^{k-1} \frac{(-1)^{n-1}}{(\beta-v)^{k-n+1}} \boldsymbol{\ell}^{(n)^{\prime}}\left[\boldsymbol{D}_{--}(\alpha, \beta)-\boldsymbol{D}_{--}(\alpha, v)-(\beta-v) \boldsymbol{I}_{--}\right. \\
& \left.-\sum_{q=1}^{k-n}(-(\beta-v))^{q} \boldsymbol{D}_{--}^{(q)}(\alpha, v)\right] .
\end{aligned}
$$

Upon letting $\beta \rightarrow v$, this leads to Eq. 32 for $n=k+1$.

To finish the proof of the theorem, we also show that:

Claim B Let $\boldsymbol{\ell}^{(1)}, \ldots, \boldsymbol{\ell}^{(m)}$ satisfy Eq. 31. If there exists some vector $\boldsymbol{\ell}$, independent of $\boldsymbol{\ell}^{(1)}, \ldots, \boldsymbol{\ell}^{(m)}$, with the property that

$$
\boldsymbol{\ell}^{\prime} \boldsymbol{D}_{--}(\alpha, v)=\boldsymbol{\ell}^{(m)^{\prime}}-\sum_{q=1}^{m} \boldsymbol{\ell}^{(q)^{\prime}} \boldsymbol{D}_{--}^{(m-q+1)}(\alpha, v),
$$

then $d \geq m+1$ and Eq. 31 holds for $n=m+1$ and $\boldsymbol{\ell}^{(m+1)}=\boldsymbol{\ell}$.

To show that Claim B holds, we suppose that $d=m$ and work towards a contradiction. The assumption $d=m$ implies that, for any vector $\boldsymbol{v}$ independent of $\boldsymbol{\ell}^{(1)}, \ldots, \boldsymbol{\ell}^{(m)}, \boldsymbol{v}^{\prime} \boldsymbol{K}_{--}^{\alpha} \neq-v \boldsymbol{v}^{\prime}+\boldsymbol{\ell}^{(m)^{\prime}}$. By definition of $\boldsymbol{M}(\alpha, \beta)$, this implies that for any $\beta \geq 0$,

$$
\boldsymbol{v}^{\prime} \boldsymbol{D}_{--}(\alpha, \beta)+(v-\beta) \boldsymbol{v}^{\prime} \boldsymbol{M}(\alpha, \beta) \neq \boldsymbol{\ell}^{(m)^{\prime}} \boldsymbol{M}(\alpha, \beta) .
$$

Using a similar argument as in the proof of Claim A, it can be seen that $\boldsymbol{\ell}^{(m)^{\prime}} \boldsymbol{M}(\alpha, v)$ equals the right-hand side of Eq. 33; this relies on the assumption that the $\ell^{(\cdot)}$ satisfy Eq. 31. A contradiction arises upon setting $v=\ell$ and letting $\beta \rightarrow v$ in the last display.

Two elements of the preceding proof deserve special attention. First, we emphasize the appealing form of the factorization Eq. 26; we encounter similar forms in the body of the paper. Another interesting point is the connection between the system 
Eq. 32 and the nonlinear matrix equation of Corollary 2.1. We use this connection to prove the following.

Corollary 8.1 The matrix equations in Corollaries 2.1 and 2.2 have a unique solution within the class of matrices with eigenvalues in $\mathcal{H}_{+}$.

Proof It suffices to prove the claim for Corollary 2.1, as the other follows similarly. Rewrite the system Eq. 32 and the equation $\boldsymbol{\ell}_{j}^{(1)^{\prime}} \boldsymbol{D}_{--}\left(\alpha, v_{j}\right)=\mathbf{0}_{-}^{\prime}$ as

$$
\begin{aligned}
\boldsymbol{0}_{d_{j-}}= & -\boldsymbol{J}_{j} \boldsymbol{L}_{j}-\boldsymbol{L}_{j} \operatorname{diag}\left(\lambda^{\alpha}\right)+\boldsymbol{L}_{j} \operatorname{diag}\left(\mu^{\alpha} \lambda^{\alpha}\right) \boldsymbol{P}_{--}^{J} \\
& +\sum_{k=0}^{d_{j}-1} e^{-v_{j} x} \frac{x^{k}}{k !}\left(v_{j} \boldsymbol{I}_{d_{j} d_{j}}+\boldsymbol{J}_{j}\right)^{k} \boldsymbol{L}_{j} \operatorname{diag}\left(\mu^{\alpha} \lambda^{\alpha}\right) \boldsymbol{P}_{-+}^{J} \boldsymbol{F}_{+-}^{\alpha}(d x),
\end{aligned}
$$

for $j=1, \ldots, s$.

In the proof Theorem 8.1, we showed that there is some $s$ such that Eq. 34 holds for a unique $d_{j}$ and unique matrices $\boldsymbol{J}_{j}$ and $\boldsymbol{L}_{j}$. The matrices $\boldsymbol{J}_{j}$ have eigenvalues in $\mathcal{H}_{+}$ and the matrices $\boldsymbol{L}_{j}$ have independent rows (uniqueness holds up to multiplication by a constant). We now argue that a solution to Eq. 34 immediately gives a solution to the equation in Corollary 2.1. To see this, stack the $s$ matrix equations of Eq. 34 into a single system, premultiply by $\boldsymbol{L}_{--}^{-1}$, note that

$$
\sum_{k=0}^{d_{j}-1} e^{-v_{j} x} \frac{x^{k}}{k !}\left(v_{j} \boldsymbol{I}_{d_{j} d_{j}}+\boldsymbol{J}_{j}\right)^{k}=e^{\boldsymbol{J}_{j} x}
$$

and use Eq. 28. The argument can also be reversed: given a solution to the equation in Corollary 2.1 with all its eigenvalues in $\mathcal{H}_{+}$, the 'building blocks' for the Jordan form must solve Eq. 34 .

\section{References}

Ahn S, Ramaswami V (2005) Efficient algorithms for transient analysis of stochastic fluid flow models. J Appl Probab 42:531-549

Arjas E, Speed TP (1973) Symmetric Wiener-Hopf factorisations in Markov additive processes. Z Wahrscheinlichkeitstheor Verw Geb 26:105-118

Asmussen S (1994) Busy period analysis, rare events and transient behavior in fluid flow models. J Appl Math Stoch Anal 7:269-299

Asmussen S (1995) Stationary distributions for fluid flow models with or without Brownian noise. Stoch Models 11:21-49

Asmussen S (2000) Ruin probabilities. World Scientific, Singapore

Asmussen S (2003) Applied probability and queues, 2nd edn. Springer, New York

Asmussen S, Avram F, Pistorius M (2004) Russian and American put options under exponential phase-type Lévy models. Stoch Process their Appl 109:79-111

Asmussen S, Kella O (2000) A multi-dimensional martingale for Markov additive processes and its applications. Adv Appl Probab 32:376-393

Bean NG, O'Reilly MM, Taylor PG (2005a) Algorithms for return probabilities for stochastic fluid flows. Stoch Models 21:149-184 
Bean NG, O'Reilly MM, Taylor PG (2005b) Hitting probabilities and hitting times for stochastic fluid flows. Stoch Process their Appl 115:1530-1556

Beneš VE (1963) General stochastic processes in the theory of queues. Addison-Wesley, Reading

Bertoin J (1996) Lévy processes. Cambridge University Press, Cambridge

Boxma OJ, Kella O, Perry D (2001) An intermittent fluid system with exponential on-times and semi-Markov input rates. Probab Eng Inf Sci 15:189-198

Chung KL (2001) A course in probability theory, 3rd edn. Academic, San Diego

Çinlar E (1972) Markov additive processes. I, II. Z Wahrscheinlichkeitstheor Verw Geb 24:85-93; 95-121

Cohen JW (1974) Superimposed renewal processes and storage with gradual input. Stoch Process their Appl 2:31-57

Dębicki K, Dieker AB, Rolski T (2007) Quasi-product forms for Lévy-driven fluid networks. Math Oper Res 32:629-647

Dębicki K, Mandjes M, van Uitert M (2007) A tandem queue with Lévy input: a new representation of the downstream queue length. Probab Eng Inf Sci 21:83-107

Dieker AB (2006) Applications of factorization embeddings for Lévy processes. Adv Appl Probab 38:768-791

Feller W (1971) An introduction to probability theory and its applications, vol II, 2nd edn. Wiley, New York

Gail HR, Hantler SL, Taylor BA (1996) Spectral analysis of M/G/1 and G/M/1 type Markov chains. Adv Appl Probab 28:114-165

Kaspi H (1982) On the symmetric Wiener-Hopf factorization for Markov additive processes. Z Wahrscheinlichkeitstheor Verw Geb 59:179-196

Kella O (2001) Markov-modulated feedforward fluid networks. Queueing Syst 37:141-161

Kella O, Whitt W (1992a) A storage model with a two-state random environment. Oper Res 40:S257S262

Kella O, Whitt W (1992b) A tandem fluid network with Lévy input. In: Queueing and related models. Oxford University Press, New York, pp 112-128

Kennedy J (1994) Understanding the Wiener-Hopf factorization for the simple random walk. J Appl Probab 31:561-563

Kroese DP, Scheinhardt WRW (2001) Joint distributions for interacting fluid queues. Queueing Syst 37:99-139

Kulkarni V (1997) Fluid models for single buffer systems. In: Frontiers in queueing. CRC, Boca Raton, pp 321-338

Kyprianou AE, Palmowski Z (2008) Fluctuations of spectrally negative Markov additive processes. In: Séminaire de probabilités vol XLI. Springer, Berlin, pp 121-135

Latouche G, Ramaswami V (1999) Introduction to matrix analytic methods in stochastic modeling. SIAM, Philadelphia

Lucantoni DM (1991) New results on the single server queue with a batch Markovian arrival process. Stoch Models 7:1-46

Miyazawa M (2004) Hitting probabilities in a Markov additive process with linear movements and upward jumps: applications to risk and queueing processes. Ann Appl Probab 14:1029-1054

Miyazawa M, Takada H (2002) A matrix exponential form for hitting probabilities and its application to a Markov-modulated fluid queue with downward jumps. J Appl Probab 39:604-618

Moler C, Van Loan C (2003) Nineteen dubious ways to compute the exponential of a matrix, twentyfive years later. SIAM Rev 45:3-49

Neuts MF (1989) Structured stochastic matrices of $M / G / 1$ type and their applications. Marcel Dekker, New York

Ney P, Nummelin E (1987) Markov additive processes. I. Eigenvalue properties and limit theorems. Ann Probab 15:561-592

Pistorius M (2006) On maxima and ladder processes for a dense class of Lévy processes. J Appl Probab 43:208-220

Prabhu NU (1998) Stochastic storage processes. Springer, New York

Preater J (1997) M/M/ $\infty$ transience revisited. J Appl Probab 34:1061-1067

Rogers LCG (1994) Fluid models in queueing theory and Wiener-Hopf factorization of Markov chains. Ann Appl Probab 4:390-413

Scheinhardt WRW, Zwart B (2002) A tandem fluid queue with gradual input. Probab Eng Inf Sci 16:29-45

Takada H (2001) Markov modulated fluid queues with batch fluid arrivals. J Oper Res Soc Jpn 44:344-365 
Takada H, Miyazawa M (2002) A Markov modulated fluid queue with batch arrivals and preemptions. Stoch Models 18:529-552

Tzenova E, Adan I, Kulkarni V (2005a) Fluid models with jumps. Stoch Models 21:37-55

Tzenova E, Adan I, Kulkarni V (2005b) A two-priority fluid flow model. Tech. Report 2005-016, EURANDOM

Zhao YQ, Li W, Braun, WJ (2003) Censoring, factorizations, and spectral analysis for transition matrices with block-repeating entries. Methodol Comput Appl Probab 5:35-58 